\title{
GABA and glycine co-transmission in the developing mouse respiratory network
}

\author{
Dissertation \\ for the award of the degree \\ "Doctor of Philosophy (Ph.D)" \\ Division of Mathematics and Natural Sciences \\ of the Georg-August-University, Göttingen \\ within the Sensory Motor Neuroscience doctoral program \\ of the Georg-August University School of Science (GAUSS) \\ Submitted by
}

Md Jamilur Rahman

from Sitamarhi, Bihar, India 


\section{Thesis Committee}

Prof. Dr. Swen Hülsmann (Supervisor)

Dept. of Neurophysiology and Cellular Biophysics,

Center for Physiology and Pathophysiology, University of Göttingen, Göttingen

Prof. Dr. Ralf Heinrich,

Dept. Cellular Neurobiology,

Schwann-Schleiden Research Centre, University of Göttingen, Göttingen

Dr. Jeong Seop Rhee,

Max Planck Institute for Experimental Medicine, Göttingen

\section{Members of the Examination Board}

Referee: Prof. Dr. Swen Hülsmann

$2^{\text {nd }}$ Referee: Prof. Dr. Ralf Heinrich

\section{Further members of the Examination Board}

Prof. Dr. Nils Brose,

Max Planck Institute for Experimental Medicine, Göttingen

Prof. Dr. Michael Müller,

Center for Physiology and Pathophysiology

Institute for Neuro- and Sensory Physiology, University of Göttingen, Göttingen

Prof. Dr. Thomas Dresbach,

Dept. of Anatomy and Embryology,

Center for Anatomy, University of Göttingen, Göttingen

Dr. Jeong Seop Rhee,

Max Planck Institute for Experimental Medicine, Göttingen

Date of oral examination: $02^{\text {nd }}$ April 2014 


\section{Declaration of originality}

I hereby declare to the best of my knowledge and belief that my thesis entitled "GABA and

glycine co-transmission in the developing mouse respiratory network" has been written independently with no other sources and aids except those acknowledged in the thesis. This thesis has not been submitted for a degree or diploma to any university or other institution.

Md Jamilur Rahman

Göttingen, January 2014 


\section{Table of contents}

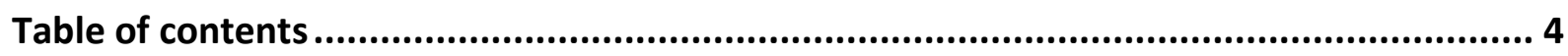

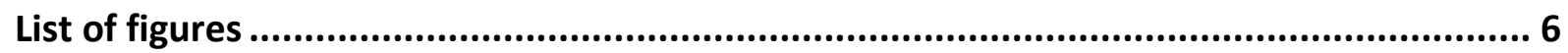

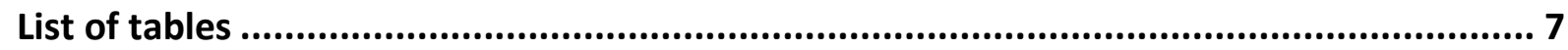

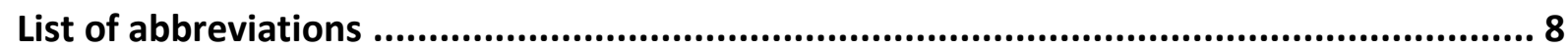

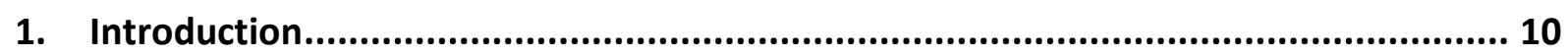

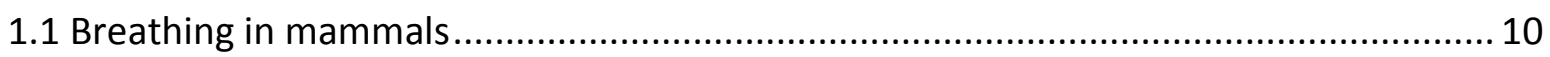

1.2 Structure and function of respiratory neuronal network ...................................... 11

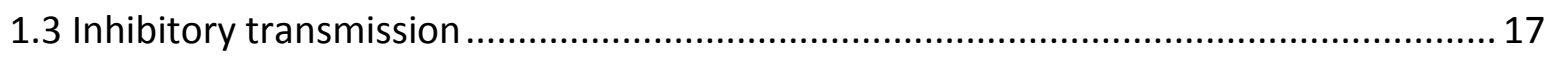

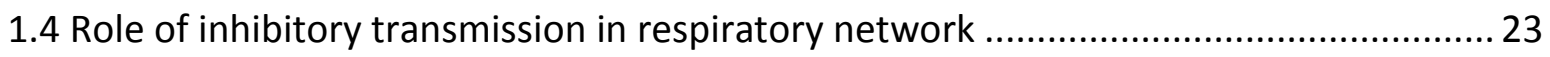

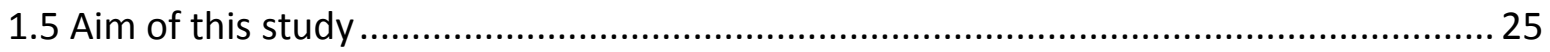

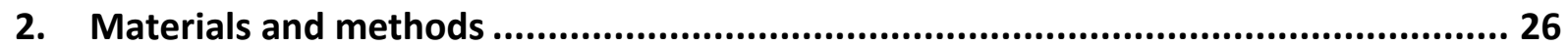

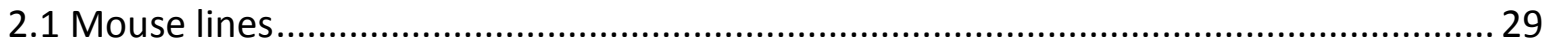

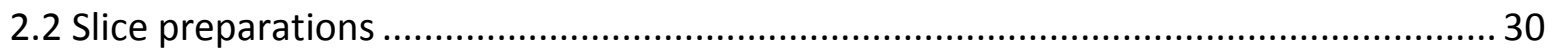

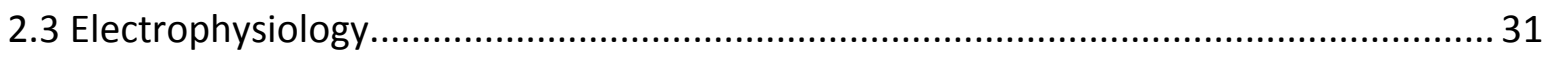

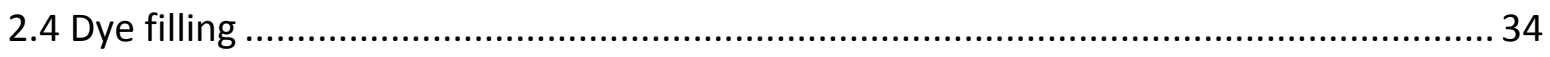

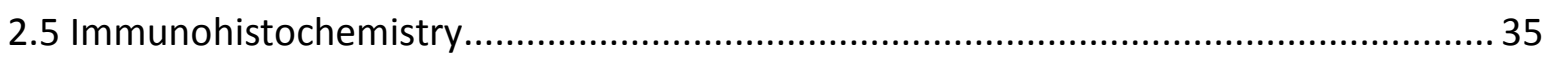

2.6 Single cell reverse transcription polymerase chain reaction .................................... 37

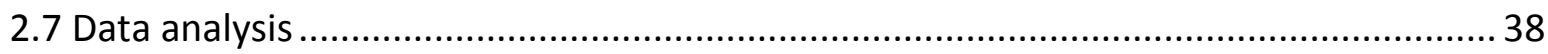

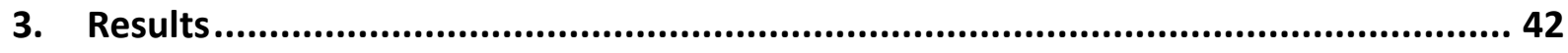

3.1 GABA and glycine co-transmission in the pre-Bötzinger complex .............................. 42

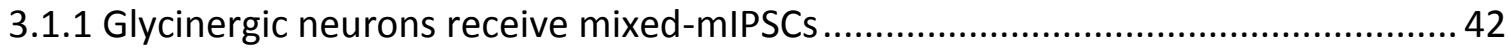

3.1.2 Rhythmic glycinergic neurons receive mixed-mIPSCs ....................................... 44

3.1.3 Glycinergic neurons co-express GABAergic and glycinergic neuronal markers ...... 46

3.2 Conditional knockout of the vesicular inhibitory amino acid transporter .................... 48 
3.2.1 Conditional VIAAT KO embryos show lethal phenotype 48

3.3.2 Respiratory rhythmic activities are present in the cVIAAT KO preBötC 51

3.2.3 VIAAT expression is reduced in medulla but not in hypothalamus 53

3.2.4 Passive and active properties of hypoglossal motor neurons 57

3.2.5 Hypoglossal motoneurons in cVIAAT KO embryos lack inhibitory transmission ..... 59

3.2.6 Glycinergic currents are reduced in CVIAAT KO hypoglossal motoneurons 61

3.2.7 Diaphragms of conditional VIAAT KO embryos are underdeveloped 63

3.3 Characterization of glycinergic neurons in the pre-Bötzinger complex 64

3.3.1 Rhythmic glycinergic neurons are primarily inspiratory. 64

3.3.2 Rhythmic glycinergic neurons receive excitatory and inhibitory inputs 66

3.3.3 Glycinergic neurons show pacemaker properties 68

3.3.4 Synaptic coupling between glycinergic neurons 70

3.3.5 Morphology of glycinergic neurons .71

4. Discussion 73

4.1 Role of co-transmission in the respiratory network. .73

4.2 Loss of inhibition affects function of hypoglossal motoneurons ...... .75

4.3 Developmental variability in co-transmission of GABA and glycine

4.4 Loss of GABAergic transmission causes omphalocele 79

4.5 Glycinergic neurons are integrated in the pre-Bötzinger complex 80

5. Summary and Conclusion 82

6. References 83

7. List of publications 99

8. Acknowledgements 100 


\section{List of figures}

Figure 1.1 Parasaggital section of adult rodent brainstem................................................ 12

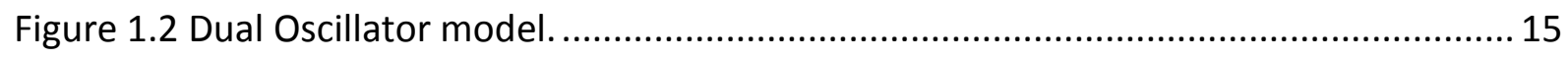

Figure 1.3 Schematic repersentation of GABA abd glycine co-transmission.......................... 22

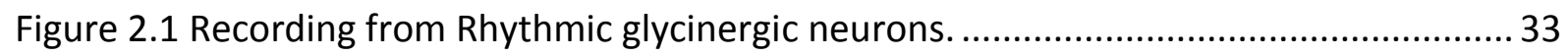

Figure 3.1 Glycinergic neurons in the preBötC receive mixed-mIPSCs................................. 43

Figure 3.2 Rhythmic glycinergic neurons in the preBötC receive mixed-mIPSCs. ................. 45

Figure 3.3 Glycinergic neurons show heterogeneous inhibitory molecular markers.............. 47

Figure 3.4 cVIAAT KO embryos showed lethal phenotype. ............................................... 49

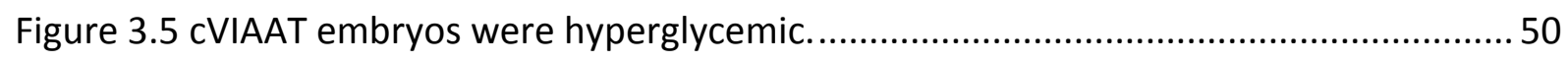

Figure 3.6 Respiratory rhythmic activity in the cVIAAT-KO slices and their control................52

Figure 3.7 VIAAT expression was dramatically reduced in cVIAAT KO hypoglossal nucleus... 54

Figure 3.8 VIAAT expression in the CVIAAT KO preBötC was reduced.....................................55

Figure 3.9 VIAAT expression in cVIAAT KO hypothalamus was not reduced...........................56

Figure 3.10 Passive properties of hypoglossal motoneurons. .............................................. 57

Figure 3.11 cVIAAT KO hypoglossal motoneurons and their controls produced APs.............. 58

Figure 3.12 cVIAAT hypoglossal motoneurons lacked inhibitory transmission...................... 60

Figure 3.13 Glycinergic postsynaptic currents are reduced in hypoglossal motoneurons...... 62

Figure 3.14 Whole-mounts neurofilament immunostaining on hemi-diaphragm................. 63

Figure 3.15 Glycinergic neurons receive the respiratory rhythmic inputs. .......................... 65

Figure 3.16 Glycinergic neurons received excitatory and inhibitory rhythmic inputs............ 67

Figure 3.17 Glycinergic neurons show pacemaker properties. ...........................................69

Figure 3.18 Paired recording from two glycinergic neurons in the preBötC. ......................... 70

Figure 3.19 Processes of glycinergic neurons in the preBötC. ............................................. 72 


\section{List of tables}

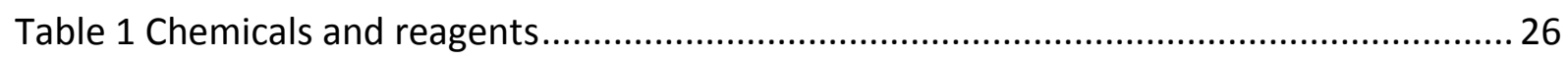

Table 2 Reagents used in singel cell reverse transcription PCR ......................................... 27

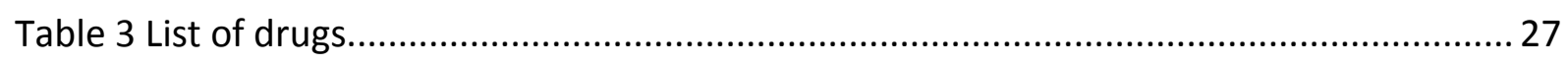

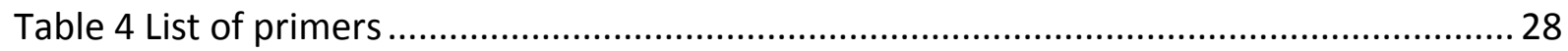




\section{List of abbreviations}

ACSF

AP

AP5

Bic

BötC

CDNA

CNQX

CNS

Ctrl

cVIAAT KO

DRC

EGFP

eIPSCs

GABA

$\mathrm{GABA}_{A} R$

GAD65

GAD67

GAT1

GlyR

GlyT1

GlyT2

GlyT2-cre

In situ

In utero

In vitro

In vivo

IPSCS

KCC2

KO
Artificial cerebrospinal fluid

Action potential

DL-2-amino-5-phosphonopentanoate

Bicuculline

Bötzinger complex

Complementary deoxyribonucleic acid

6-cyano-7-nitroquinoxaline-2,3-dione

Central nervous system

Control

Conditional VIAAT knockout

Dorsal respiratory column

Enhanced green fluorescent protein

Evoked inhibitory postsynaptic currents

$\gamma$-aminobutyric acid

$\mathrm{GABA}_{\mathrm{A}}$ receptor

Glutamate decarboxylase 65

Glutamate decarboxylase 67

GABA transporter 1

Glycine receptor

Glycine transporter 1

Glycine transporter 2

Glycine transporter 2 cre-recombinase

Being in original position or unmoved

In the uterus

In an artificial environment outside the living organism

In the living organism

Inhibitory postsynaptic currents

Potassium chloride cotransporter

Knockout 
mIPSCs

mixed-mIPSCs

$\mathrm{mM}$

MNs

NKCC

NKCC1

NTS

P

PCR

preBötC

pFRG

$\mathrm{R}_{\text {in }}$

PRG

RT-PCR

SIPSCS

SC-RT-PCR

Stry

SVs

TTX

VGAT

VIAAT

VRC

¿EPF
Miniature inhibitory postsynaptic currents

Mixed miniature inhibitory postsynaptic currents

Millimolar

Motoneurons

$\mathrm{Na}^{+}-\mathrm{K}^{+}-2 \mathrm{Cl}^{-}$cotransporter

Sodium potassium chloride cotransporter

Nucleus of the tractus solitarius

Postnatal day

Polymerase chain reaction

Pre-Bötzinger complex

Para facial respiratory group

Input resistance

Pontine respiratory group

Reverse transcriptase polymerase chain reaction

Spontaneous inhibitory postsynaptic currents

Single cell reverse transcriptase polymerase chain reaction

Strychnine

Synaptic vesicles

Tetrodotoxin

Vesicular GABA transporter

Vesicular inhibitory amino acid transporter

Ventral respiratory column

Sum of exponential product function 


\section{Introduction}

\subsection{Breathing in mammals}

Breathing is a vital behavior, which starts at birth and continues till death. Respiration is required to maintain, a physiological level of $\mathrm{O}_{2}$ and $\mathrm{CO}_{2}, \mathrm{pH}$ in the blood and to control body thermoregulation. Before birth, these parameters are controlled through the placenta of the mother but immediately after birth, the respiratory circuitry in brainstem and lungs of the newborn take over this responsibility. If a neonate manages this critical moment successfully, he/she can survive, while those who fail die. This means that the respiratory neurons, muscle, and organs responsible for respiration, have to be functional before they are needed for survival. Furthermore, breathing has to be robust, yet flexible, as it gets modulated in varying physiological situations that require changes of breathing during physical exercise and sleeping to accurately meet the physiological demand. The respiratory rhythm changes during behaviors such as speech, chewing or swallowing (Feldman \& Del Negro, 2006). Respiratory-like movements already appear in utero shortly after the onset of fetal movements in mice at E14.5 (Viemari et al., 2003) and in humans at 10-12 weeks of gestation (de Vries et al., 1982).

Normal breathing is achieved with a precise tuning of the respiratory rhythm generator (RRG) and coordinated activity of respiratory muscles including those of the upper respiratory tract. Principally, a breathing cycle starts with inspiration, followed by postinspiration (PI) and ends with expiration. Inspiration is generated in brainstem and coordinated by simultaneous contraction of diaphragm and external intercostal muscles. The movement of diaphragm reduces the thoracic pressure that forces air to enter the lungs, and thereby allows gaseous exchange $\left(\mathrm{O}_{2}\right.$ and $\left.\mathrm{CO}_{2}\right)$. Expiration is a passive process which is achieved through same respiratory tract by recoiling of diaphragm and lungs. However, in forced expiration, internal intercostal muscles and abdominal muscles are also involved (Feldman et al., 2003; Feldman \& Del Negro, 2006). The neuronal network, which controls inspiratory and expiratory motor output, together known as the central pattern generator (CPG). The CPG is located in the medulla oblongata and pons. 
Respiration can be disturbed with deletion of a single gene, for an example, potassiumchloride cotransporter (KCC2) (Hubner et al., 2001), v-aminobutyric acid (GABA) synthesizing enzyme (GAD67) (Asada et al., 1997), glycine transporter 1 (GlyT1) (Gomeza et al., 2003a), vesicular GABA transporter (VGAT/VIAAT) (Fujii et al., 2007; Saito et al., 2010), Pbx3 (Rhee et al., 2004), or Na-K.ATPase (Ikeda et al., 2004). Respiration is also partially or completely ceased in several diseases and syndromes like Rett syndrome (Amir et al., 1999), congenital central hypoventilation syndrome (CCHS) (Chen \& Keens, 2004; Gozal, 2004; Rhee et al., 2004), sudden infant death syndrome (SIDS) (Weese-Mayer et al., 2003; Garcia et al., 2013), amyloid lateral sclerosis (ALS) (Goulon \& Goulon-Goeau, 1989), Parkinson's disease (Maclntosh, 1977), and multiple systems atrophy (MSA) (McDonald, 1974).

\subsection{Structure and function of respiratory neuronal network}

The respiratory network is bilaterally arranged in a rostro-caudal direction from pons to medulla (Smith et al., 2007; Koizumi et al., 2008; Smith et al., 2013). Respiratory neurons across pons and medulla are categorized broadly, but not limited, to three groups: pontine respiratory group (PRG), ventral respiratory column (VRC) and dorsal respiratory column (DRC) located in medulla (Fig. 1.1b). Respiratory neurons interact within these regions to produce the final output pattern which is transmitted through premotor networks to cranial and spinal motoneurons (Rybak et al., 2007; Alheid \& McCrimmon, 2008; Smith et al., 2013). The most important parts of the RRG are present in the VRC that is located ventrolaterally in the medulla, particularly ventral to the nucleus ambiguus (Amb), and extend from the caudal end of the facial nucleus (FN) to the rostral end of cervical spinal cord.

\section{pre-Bötzinger complex}

In the VRC, the pre-Bötzinger complex (preBötC) is located just caudal to the BötC (see below) and is accepted to be the kernel for respiratory rhythm generation. For the first time, the preBötC was identified in ventrolateral medulla (VLM) by micro sectioning of neonatal rat brainstem while recording the cranial and spinal motor output (Smith et al., 1991). Subsequently, the preBötC was identified in cat (Schwarzacher et al., 1995) and mice (Ramirez et al., 1996) as the site for the RRG. Later, it was also shown in vivo that the preBötC is responsible for breathing (Ramirez et al., 1998). 

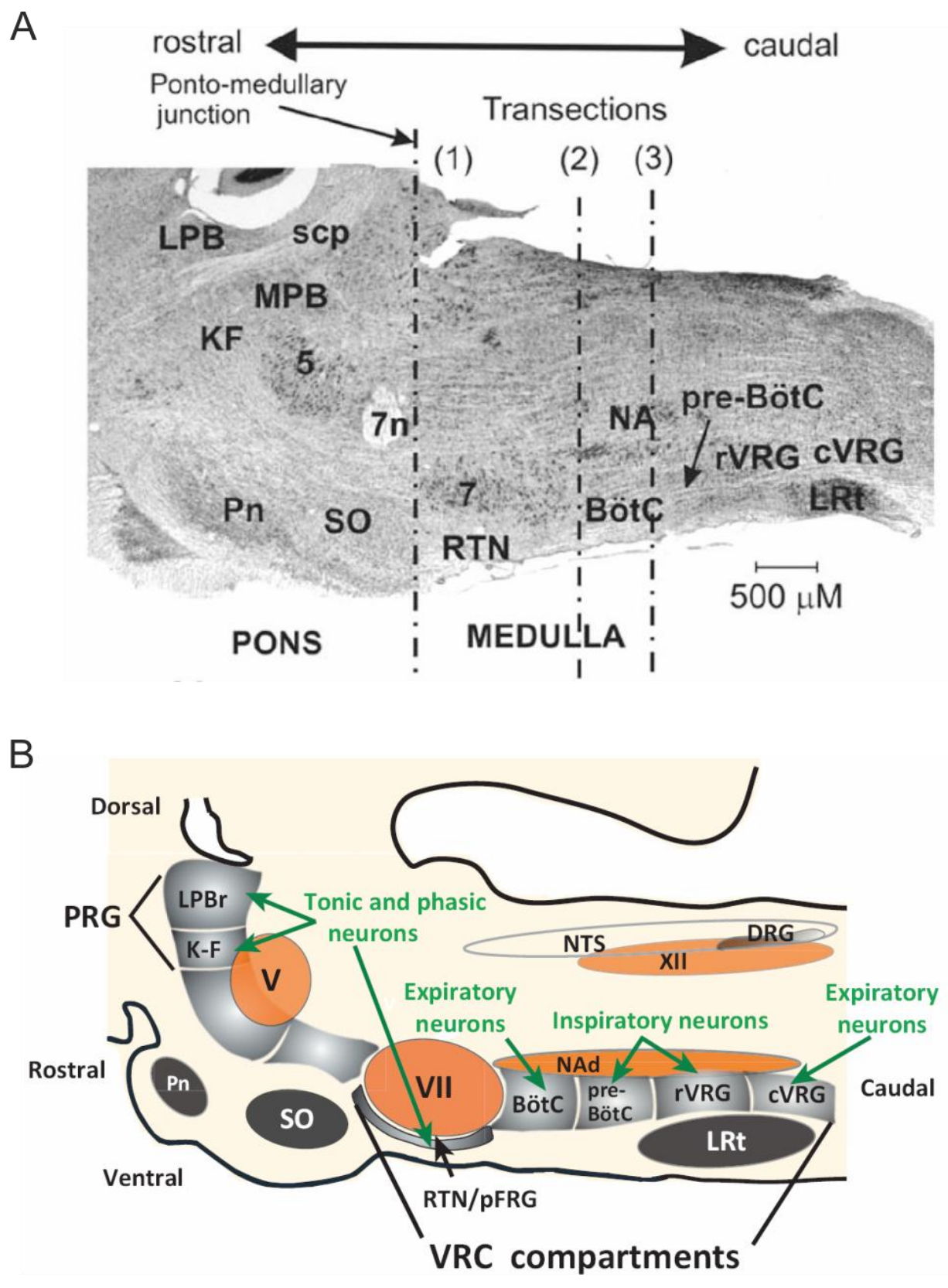

Figure 1.1 Parasaggital section of adult rodent brainstem.

Parasagittal section of adult rodent brainstem shows different components of respiratory CPG network. Three transection cuts (dotted lines) are used during experimental preparation to study different phases of respiratory rhythm. (B) A sketch describes major respiratory nuclei in a parasagittal section through one side of rodent brainstem. Abbreviations area here: 5(or V)-trigeminal nucleus; 7(or VII)facial nucleus; 7n-facial nerve; XII-hypoglossal nucleus; BötC-Bötzinger Complex; cVRG-caudal ventral respiratory group; DRC-dorsal respiratory column; KF-Kölliker Fuse nucleus; LPB-lateral parabrachial nucleus; LRt: lateral reticular nucleus; MPB-medial parabrachial nucleus; NA-nucleus ambiguus; NAdnucleus ambiguous dorsal division; NTS-nucleus of the tractus solitarius; PB-parabrachial nuclei; Pnpontine nuclei; preBötC-pre-Bötzinger complex; PRG-pontine respiratory group; RTN-retrotrapezoid nucleus; rVRG-rostral ventral respiratory group; scp-superior cerebellar peduncle; SO-superior olive. Figure 1.1A is adapted from Rybak et al, 2007 and figure 1.1B is adapted from Smith et al, 2013 with respective author's and Elsevier permission. 
The preBötC contains glutamatergic, GABAergic and glycinergic neurons (Kuwana et al., 2006; Wallen-Mackenzie et al., 2006a; Winter et al., 2009). A subgroup of glutamatergic neurons in the preBötC, which expresses neurokinin 1 receptor (NK1R) (Gray et al., 1999) and the peptide somatostatin (SST) (Stornetta et al., 2003), is responsible for the respiratory rhythm generation. These NK1R and SST expressing and other glutamatergic neurons, in the VLM are derived from a developing brain homeobox 1 (DBX1) expressing progenitor cells (Gray et al., 2010). The loss of DBX1 removes all glutamatergic cells from VLM including the preBötC which are responsible for RRG both in vivo and in vitro (Gray et al., 2010). The exact anatomical location and functions of the preBötC became more evident from subsequent publications which showed that neurons in the preBötC are responsible for RRG, by lesion/ablation studies (Gray et al., 1999; Gray et al., 2001; Tan et al., 2008), anatomical studies (Guyenet \& Wang, 2001; Wang et al., 2001; Tan et al., 2010), developmental studies (Champagnat et al., 2009; Bouvier et al., 2010; Gray et al., 2010), and optogenetics studies (Pagliardini et al., 2011).

\section{Parafacial respiratory group}

A second group of neurons, ventrolateral to the facial nucleus and close to the ventral surface, is referred as the parafacial respiratory group (pFRG). In the neonatal pFRG, neurons are rhythmically active and their rhythmic activity is well synchronized and precede $500 \mathrm{~ms}$ from phrenic nerve activity in en-bloc preparation and they are also called as pre-inspiratory (PI) neurons (Onimaru \& Homma, 2003). The rhythmic activity and location of the pFRG is also confirmed by another publication which showed the PFRG rhythmic activity in embryonic rodent en-bloc and rhythmic slices preparations (Thoby-Brisson et al., 2005). The silencing of the pFRG (by knocking out a hindbrain transcription factor Egr2) causes an abnormal respiratory rhythm (Thoby-Brisson et al., 2009). The intrinsic rhythmic frequency of the pFRG is slower compared to rhythmic frequency recorded from either hypoglossal or phrenic rootlet (Thoby-Brisson et al., 2005). In the adult animals, the pFRG has been suggested to be involved in expiration (Janczewski \& Feldman, 2006a) and forced expiration (Feldman \& Del Negro, 2006). The rhythmic activity in the pFRG starts earlier (E14.5) than the rhythmic activity in the preBötC (E15.5) and therefore it is also hypothesized that the pFRG initially entrain and then couple with the preBötC to produce normal respiratory 
rhythmic activity (Thoby-Brisson et al., 2009). This leads to a new concept of two oscillators (the pFRG and the preBötC), thus to the dual organization of the RRG (Fortin \& ThobyBrisson, 2009; Thoby-Brisson et al., 2009). The concept of two oscillators in the neonatal rodent is supported by computational modeling and experiments by several groups (Onimaru et al., 1990; Onimaru \& Homma, 2003; Janczewski \& Feldman, 2006a; b; Wittmeier et al., 2008; Lal et al., 2011).

\section{Bötzinger complex}

The Bötzinger complex (BötC), the most rostral part of the VRC, was shown in the cat as a group of expiratory neurons located ventral to the rostral portion of the nucleus ambiguus (Amb) (Ezure et al., 1988). Among expiratory neurons, the BötC also contains postinspiratory neurons (Smith et al., 2007), which inhibit inspiratory neurons in other parts of the respiratory circuitry (Ezure et al., 2003a). The BötC expiratory-augmenting neurons project to the spinal cord reaching very close to phrenic motoneurons (Tian et al., 1998). A subgroup of the BötC neurons sends axons rostral to the facial nucleus and also appear to target the pons (Ezure et al., 2003b). Functionally, the BötC is involved in changing the phase between inspiration and expiration. 


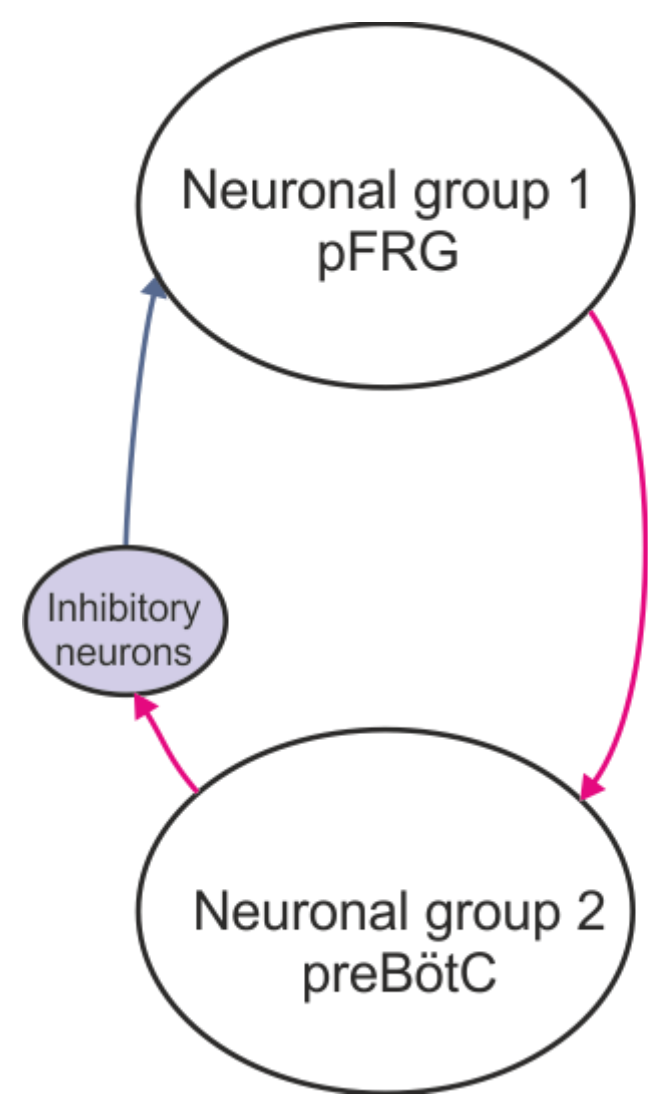

\section{$\longrightarrow$ Excitatory connection Inhibitory connection}

Figure 1.2 Dual Oscillator model.

Neuronal group 1 (the pFRG) provides excitatory synaptic inputs to neuronal group 2 (the preBötC) which then provides excitatory synaptic inputs to a group of inhibitory neurons. These inhibitory neurons provide inhibitory inputs to neuronal group 1.

\section{Ventral Respiratory Group}

Caudal to the preBötC, the ventral respiratory group (VRG) continues to the rostral end of spinal cord. Anatomically, the VRG is further divided into two parts, rostral VRG (rVRG) and caudal VRG (cVRG). The rVRG is located caudal to the preBötC and it consists of premotoneurons connected to inspiratory motoneurons in spinal cord (Alheid \& McCrimmon, 2008). It receives excitatory inputs from the preBötC and inhibitory inputs from the BötC. The CVRG, which is located caudal to the rVRG, contains bulbospinal premotoneurons which innervate the expiratory motoneurons the in spinal cord. The rVRG controls the inspiratory pattern whereas the cVRG controls the expiratory respiratory pattern. 


\section{Nucleus of the tractus solitarius}

The respiratory neurons in the nucleus of the tractus solitarius (NTS) receive feedback inputs from afferent fibres originating in lower and upper respiratory track related to chemo and mechanical senses (Paton et al., 1994; Boscan et al., 2002). These feedbacks are critical in integrating breathing with other physiological behaviours like emotion and sleeping.

\section{Hypoglossal nucleus}

The hypoglossal motoneurons ( $\mathrm{HMn}$ ) are located below the fourth ventricle and innervate tongue muscles, which are involved in maintaining the patency of tongue during respiration, speech, swallowing, and mastication (Mu \& Sanders, 2010). The patency of pharyngeal lumen is maintained by four main muscles, of which, tongue muscles are innervated by HMn while the hyoid muscles are innervated by neurons located ventral to $\mathrm{HMn}$ and soft palate muscles are innervated by neurons located dorsolateral to HMn and Amb (Sokoloff \& Deacon, 1992). The respiratory neurons in hypoglossal nucleus receive direct inputs from the preBötC and is often recorded to monitor the respiratory activity in rhythmic slice preparation (Tan et al., 2010). HMn receive both GABAergic and glycinergic inhibitory terminals (Aldes et al., 1988); of which, GABAergic inhibitory signals come mainly from the nucleus of Roller, which is located ventral to HMn (van Brederode et al., 2011). It has also been shown that hypoglossal motoneurons receive simultaneous GABA and glycine inputs which is likely due to co-release of GABA and glycine (O'Brien \& Berger, 1999). A disturbance in the HMs is thought to be involved in the pathogenesis of obstructive sleep apnea (Horner, 2007).

\section{Pontine nuclei}

The pontine respiratory group (PRG) is located in the rostral dorsolateral side of the pons. The respiratory neurons in the PRG are present mainly in parabrachial (PB) nuclei and Kölliker-Fuse (KF), which regulate the inspiratory-expiratory phase transition (Dutschmann \& Herbert, 2006). The lateral PB influences an inspiratory facilitation while the medial PB and the KF facilitate largely an expiratory response as shown by chemical and electrical experiments (Lara et al., 1994; Spyer, 2009). The KF receives and sends signals to the NTS and to the BötC (Smith et al., 2013). 


\subsection{Inhibitory transmission}

The balance between excitatory and inhibitory transmission needs to be maintained for a normal physiological state, otherwise it could result into pathophysiological and neurodegeneration diseases. In addition to maintaining the homeostasis, both GABA and glycine have many critical roles in the cell signaling of the CNS and non-neuronal tissues (den Eynden et al., 2009; Chalphin \& Saha, 2010). They also play a role in the development and differentiation of the CNS (Chalphin \& Saha, 2010; Tatetsu et al., 2012). Therefore, the disturbance in GABA transmission is ascribed to many neurological diseases including epilepsy (Freichel et al., 2006) and general anxiety disorder (Kosel et al., 2004). The importance of inhibitory transmission in respiratory rhythm patterning is described in section 1.4 .

\section{Synthesis of inhibitory transmitters in the presynaptic terminal}

GABA is synthesized from glutamate either in the cell body or presynaptic terminal, with the help of glutamic acid decarboxylase (GAD), which exists in two main isoform; GAD67 and GAD65 (Lernmark, 1996; Bosma et al., 1999) and two smaller isoform; GAD25 and GAD44 (Szabo et al., 1994). Glycine is synthesized in the body from serine by serine hydroxymethyltransferase (SHMT) (Hernandes \& Troncone, 2009).

\section{Packaging of inhibitory transmitters into synaptic vesicle}

The neurotransmitters are packaged into a small clear vesicle (SCV), commonly called as "synaptic vesicle" (SV), before they are docked to the cell membrane of presynaptic nerve terminal. The vesicular GABA transporter (VGAT) was shown to fill GABA into synaptic vesicles (McIntire et al., 1997). In the same year, the co-localization of VGAT with GAD67 and with GlyT2 (a glycine neuronal transporter) were shown which lead to a hypothesis that the VGAT could fill both GABA and glycine in SVs, therefore, the VGAT was named vesicular inhibitory amino acid transporter (VIAAT) (Sagne et al., 1997). Subsequently many groups confirmed that VIAAT fills both GABA and glycine into SVs (Chaudhry et al., 1998; Dumoulin et al., 1999; Chessler et al., 2002; Supplisson \& Roux, 2002; Ebihara et al., 2003; Gammelsaeter et al., 2004). 


\section{Action of inhibitory transmitters on postsynaptic receptors}

After arrival of an action potential (AP) at a presynaptic terminal, inhibitory transmitters are released into a synaptic cleft and exert their action through GABAergic receptors (GABAR) and/or glycinergic receptors (GlyR) which are expressed on the cell membrane of a postsynaptic neuron. The GABAR are classified into different classes $\left(G A B A_{A} R, G A B A_{B} R\right.$, and $G_{A B A} R$ ); among them, $G A B A_{A} R$ and $G A B A_{C} R$ are ionotropic while $G A B A_{B} R$ is metabotropic. $\mathrm{GABA}_{A} R$ forms pentameric chloride channels using different combination of its subunits $(\alpha$, $\beta, \gamma$ ); the most common combination is $2 \alpha$-subunits, $2 \beta$-subunits, and $1 \gamma$-subunit (Farrar et al., 1999; Cherubini \& Conti, 2001). Although different types and subunits of $G A B A_{A} R$ provide higher flexibility in GABA mediated inhibition, but $\beta 3$-subunit inactivation makes the receptor non-functional (Culiat et al., 1995). Inhibitory transmission in brainstem, spinal cord, and retina is mainly mediated through GlyR (Sato et al., 1991). GlyR is also a pentameric chloride channel forms by a different combination of its subunits ( $\alpha 1-4$ and $\beta$ ); the most common in adult CNS is $3 \alpha: 2 \beta$ (Langosch et al., 1988). The expression and combination of its subunits depends on age and tissues of an animal (Lynch, 2004). While homomeric GlyR are reported only at embryonic stage, heteromeric GlyR are expressed in embryonic as well as postnatal stages. GlyR are reported to be highly concentrated in the grey matter and the hypoglossal nucleus (Graham et al., 1967; Hernandes \& Troncone, 2009).

\section{Recycling of inhibitory neurotransmitters by membrane transporters}

GABA is regulated at synapses through different GABA transporters (GAT), which consist of four types GAT1, GAT2, GAT3, and betaine-GABA transporter1 (Borden et al., 1995). Among them, GAT1 is commonly expressed by neurons and most abundantly present in the CNS (Borden et al., 1995). Glycine is regulated at synapse through either glycine transporter 1 (GlyT1) or glycine transporter 2 (GlyT2) or both. The GlyT1 has three splice variants (GlyT1a, GlyT1b, GlyT1c) while GlyT2 has none (Borowsky et al., 1993; Jursky \& Nelson, 1996). In the respiratory network, GlyT1 is primarily expressed on astroglial cells (Szoke et al., 2006), which regulates the extracellular concentration of glycine at synapses (Gomeza et al., 2003a). GlyT2 is expressed on neurons (Zafra et al., 1995; Jursky \& Nelson, 1996; Gomeza et al., 2003b) and is responsible for maintaining the glycine concentration in the presynaptic terminal (Gomeza et al., 2003b; Latal et al., 2010). 


\section{Inhibitory transmission switch from excitatory to inhibitory during development}

GABA and glycine were discovered as inhibitory transmitters in the beginning, but now it is known that they can be both excitatory in immature neurons and inhibitory in mature neurons (Cherubini et al., 1991; Flint et al., 1998; Ben-Ari, 2001). The depolarizing action of GABA and glycine in immature oligodendrocytes progenitor cells causes intracellular calcium influx (Belachew et al., 2000), that may be necessary for the development and maturation of various synapses including inhibitory synapses (Kneussel \& Betz, 2000; Aguayo et al., 2004). In rodent, GABA and glycine are already inhibitory around E19 (Ren \& Greer, 2006) and many reports confirm the inhibitory action of GABA and glycine at P0 (Feng et al., 1998; Hubner et al., 2001; Gomeza et al., 2003a; Gomeza et al., 2003b).

In general the switch from depolarizing to hyperpolarizing actions of GABA or glycine depends on the intracellular chloride concentration in a neuron. GABA and glycine cause depolarization in immature neurons which have high chloride concentration (Ben-Ari, 2001; Gaiarsa et al., 2002) and hyperpolarization in mature neurons which have low chloride concentration. The intracellular chloride concentration depends on the expression of different $\mathrm{K}^{+} / \mathrm{Cl}^{-}$co-transporters (KCCs). The high intracellular chloride concentration is due to higher expression of sodium-potassium-chloride cotransporter 1 (NKCC1) whereas low chloride concentration is due to higher expression of potassium-chloride cotransporter 2 (KCC2) (Plotkin et al., 1997; Rivera et al., 1999; Ben-Ari, 2002). The chloride concentration can also be regulated by the endogenous modulator brain-derived neurotrophic factor (BDNF) by down regulation of KCC2 (Rivera et al., 2002). A second developmental change concerns the composition of postsynaptic receptors. During development, subunit composition of GlyR is changed from $\alpha 2$ in prenatal to $\alpha 1$ in adult while $\beta$ remains unchanged (Legendre, 2001; Lynch, 2004; 2009). Furthermore, a GlyR is expressed as both homomeric and heteromeric channels in immature neurons, whereas it is expressed only as heteromeric channel in mature neurons (Hoch et al., 1989; Aguayo et al., 2004). Interestingly, the cluster size of the GlyR increases during same developmental change too. Similar changes also occur in composition of $\mathrm{GABA}_{A} \mathrm{R}$ (Fritschy et al., 1994; Liu \& Wong-Riley, 2004). 


\section{Glycinergic transmission increases during development}

Although both GABAergic and glycinergic transmissions are present in the whole CNS, GABAergic transmission is dominated in the forebrain whereas glycinergic transmission is dominated in the hindbrain (Berger, 2011). The relative contribution of GABA and glycine inhibitory transmission changes during development (Singer \& Berger, 2000). In spinal motoneurons, the glycine-mediated component is increased several folds in neonate compared to their embryonic stage (Gao \& Ziskind-Conhaim, 1995), while mixed-mIPSCs resulting from simultaneous activation of $\mathrm{GABA}_{A} \mathrm{R}$ and GlyR remain the same (Gao et al., 2001). This developmental change may be due to an increase in the cluster-size and affinity of GlyR with glycine as shown in rat spinal cord and brainstem (Aguayo et al., 2004). During development, a decrease in GABA mediated component and an increase in glycine mediated component are also shown in lateral superior Olive (LSO) of gerbil and rat by electrophysiology and immunohistochemical experiments respectively (Kotak et al., 1998; Nabekura et al., 2004).

\section{Cotransmission}

Chemical transmission as the mode of communication between neurons was reported for the first time by Sir Henry Dale (Dale, 1914). Then, neurons were classified based on their neurotransmitter release and also how they act on the postsynaptic cell. Until 1950, it was assumed that neurons in peripheral nervous system communicate through either acetylcholine or epinephrine or nor-epinephrine only and that their actions can be either excitatory or inhibitory depending on the postsynaptic side (Dale, 1935). The principle "one neuron-one transmitter", was construed by Prof. Eccles in 1957 based on Dale's original article "A neuron is a metabolic unit and operates at all its synapses by the same chemical transmission mechanisms" (Dale, 1935). However, in 1960s Eccles's principle of "one neuron-one transmitter" disproved after the publication where adrenergic neurons of the rat pineal gland were positive for 5-hydroxytryptamine (5-HT) (Owman, 1964). This was the first description of two transmitters in the same nerve terminal. Furthermore, the direct evidence for coexistence of two neurotransmitters (acetylcholine and noradrenalin) was shown in the sympathetic nerve terminal by co-culturing sympathetic neurons with cardiac 
myocytes (Patterson \& Chun, 1974; Furshpan et al., 1976). The first example of a co-release was reported in the electric organ of Torpedo where it was shown that ATP is co-released with ACh (Whittaker et al., 1972). Subsequently, other groups also reported the co-release of peptides such as Substance $\mathrm{P}$, Somatostatin, Bradykinin and Endorphin with ACh (Burnstok, 1972; Agranoff 1975) as reported by (Burnstock, 1976).

\section{Cotransmission of GABA and glycine}

The co-localization of GABA and glycine in a single presynaptic nerve terminal was shown by Immunohistochemistry in a subset of retinal neurons of tiger salamander (Yazulla \& Yang, 1988). This finding was further supported by another study which showed the presence of both GABA and glycine in the same synaptic vesicle of a nerve terminal isolated from rat medulla via immunogold labeling (Burger et al., 1991). Supporting the possibility of cotransmission, another group showed that the majority of cultured rat spinal neurons are activated by both GABA and glycine meaning that the same neuron express both $G A B A_{A} R$ and GlyR (Smith et al., 1989). The co-localization of GABA and glycine in the presynaptic terminal and the co-expression of $G_{A B A} R$ and GlyR on the postsynaptic side at the same synapse is shown in an adult rat spinal cord (Todd et al., 1996) which is another clear indication that co-transmission of GABA and glycine can happen in spinal cord. 


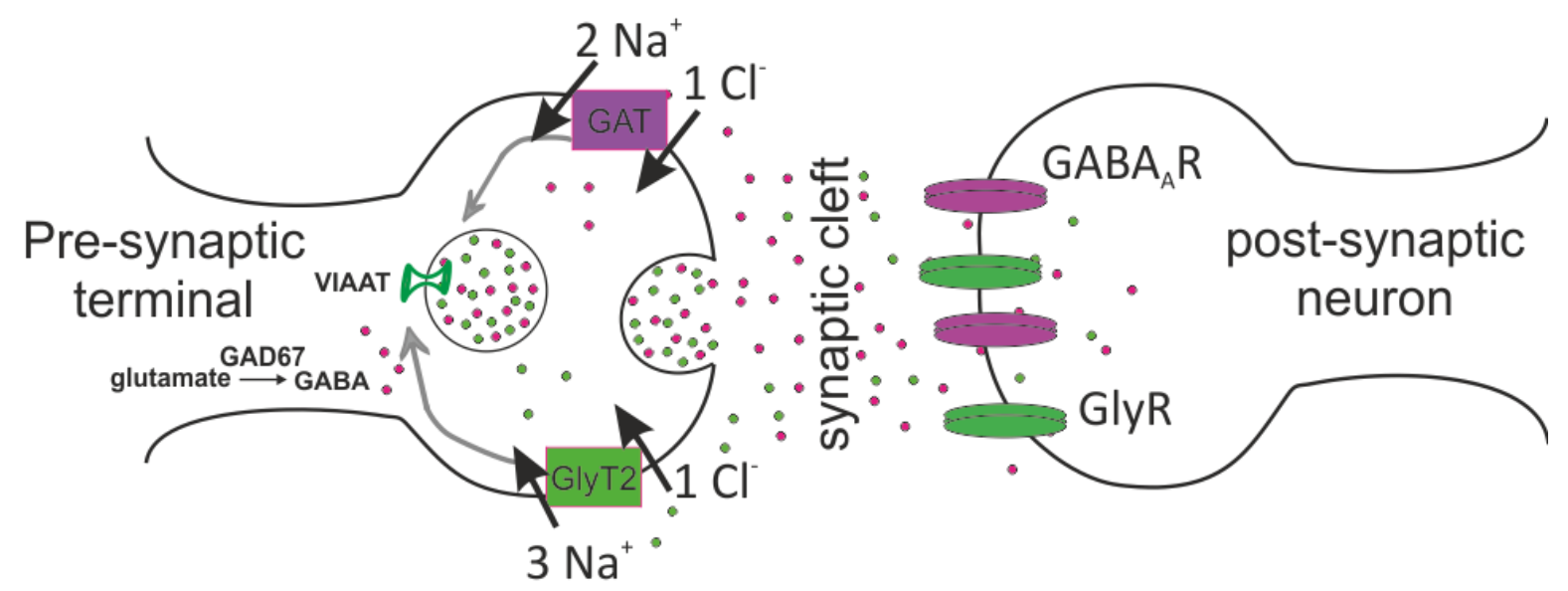

Figure 1.3 Schematic repersentation of GABA abd glycine co-transmission.

GABA is synthesized from glutamate with the help of GAD67 and transported through GAT and glycine is transported through GlyT2 in the presynaptic terminal. Both GABA and glycine are filled into SV with the help of VIAAT. GABA and glycine filled SV fuses to presynaptic membrane upon arrival of $A P$ and then release its content into synaptic cleft to act on postsynaptic $G A B A_{A} R$ and GlyR.

Finally, the co-release of GABA and glycine is shown by paired electrophysiological recording at the central synapse between interneuron and motoneuron in rat spinal cord slices (Jonas et al., 1998). Another group showed that markers for both GABA and glycine co-localize at presynaptic and their receptors on postsynaptic side at the same synapses in the primary culture as well as in spinal cord slices by immunohistochemistry (Dumoulin et al., 1999). The co-release was further shown in other brain regions including neonatal rat hypoglossal motoneurons (O'Brien \& Berger, 1999), rat cerebellum (Dumoulin et al., 2001), rat abducens motoneurons (Russier et al., 2002), rat midbrain, pons and cerebellum (Tanaka \& Ezure, 2004). It has been postulated that co-release of GABA and glycine controls precisely a highly complex behavior such as auditory processing (Awatramani et al., 2005; Lu et al., 2008; Singer, 2008). These publications suggest that co-release is a general phenomenon rather than an exception. 


\subsection{Role of inhibitory transmission in respiratory network}

Inhibitory transmission in respiratory network has been studied for last couple of decades, which highlight its role on respiratory rhythmic activity through wide range of experiments. These studies are broadly classified into two sections; pharmacological and genetics.

\section{Pharmacological studies}

The inhibitory transmission is important for all coordinated motor outputs including respiration. In neonate brainstem-spinal cord preparation, blocking of $G_{A B A} R$ and $G$ lyR by bicuculline (Bic) and strychnine (Stry) as well as low chloride concentration in the bath, reduce the phasic inhibition and increase the firing frequency of pre-inspiratory neurons during inspiration (Onimaru et al., 1990). However, the blockade of GABAR and GlyR do not stop pre-inspiratory rhythm generation in RVLM, which suggest that synaptic inhibition is not required for respiratory rhythm generation but for its modulation and patterning (Onimaru et al., 1990).

In adult rat brainstem-spinal cord preparation, both agonists and antagonists of $G A B A_{A} R$ and GlyR were applied through arterially perfusion to monitor their effect on the motor output of two important respiratory nerves, phrenic and hypoglossal. The agonists (GABA, glycine and muscimol) reduce, while antagonists (picrotoxin, bicuculline, strychnine and phaclofen) increase both frequency and amplitude of inspiratory bursts (Hayashi \& Lipski, 1992). These results were further corroborated by another study which showed the blockade of GlyR by strychnine also increases the frequency of rhythmic bursts recorded from hypoglossal rootlet in rhythmic slice made from neonatal and adult mice (Ramirez et al., 1996). Subsequently, another study showed that reciprocal inhibition of expiratory neurons in the preBötC was primarily mediated by glycinergic transmission and also confirmed that strychnine and bicuculline increase the frequency (even induce seizure) of hypoglossal rootlet bursts in neonate rat medullary slice (Shao \& Feldman, 1997). The role of inhibitory transmission in respiration was also shown in the working heart-brainstem preparation (most close to in vivo), where the 3-phase respiratory rhythm (inspiration, post inspiration, and expiration) after systemic application of strychnine changes to 2-phase respiratory rhythm (inspiration and expiration) by merging of postinspiratory phase into inspiratory phase with increase in rhythmic frequency (Busselberg et al., 2001). The systemic application 
of strychnine would have blocked all glycinergic transmission in the network of workingheart-brainstem preparation which may have resulted complete absence of the postinspiratory phase. Indeed that was the case, because selective blocking (through viral delivery of allatostatin receptor under GlyT2-promoter and subsequent application of allatostatin) of glycinergic neurons in the BötC, only decreases but does not eliminate the postinspiratory component (Abdala AP, 2010). In contrast, the bilateral microinjection of bicuculline and strychnine in the BötC strongly reduces the respiratory activity even causes apnea, whereas bicuculline injection in the preBötC causes a small decrease in respiratory frequency and increase with strychnine in anesthetized, vagotomized, paralyzed and artificially ventilated rabbits (Bongianni et al., 2010). Contradicting above findings, a recent study suggests that the rhythmic activity does not change after blocking the inhibitory transmission except the lung's inflation induced inhibitory reflex (Breuer-Hering) is suppressed (Janczewski et al., 2013).

\section{Genetic studies}

GABA is mainly synthesized by either GAD65 or GAD67 enzymes. Knocking out (KO) of GAD65 in mice does not change respiration, locomotion, and GABA level in CNS, but the GAD65 KO mice become susceptible to seizures (Asada et al., 1996). However, the GAD67 KO mice have severe phenotype including disturbance in respiratory pattern, cleft palate, and the mice die during "first morning after birth" (Asada et al., 1997; Condie et al., 1997; Kuwana et al., 2003; Tsunekawa et al., 2005; Fujii et al., 2007; Oh et al., 2010). In addition, GAD67 KO mice also show a decrease in respiratory and increase in non-respiratory muscles (Fogarty et al., 2013a). Considering the function of VIAAT (the only known vesicular transporter for GABA and glycine), one would expect that its removal would affect phenotype severely. Exactly this is shown in the VIAAT KO mouse embryos that do not breathe (Saito et al., 2010), do not show any phrenic activity (Fujii et al., 2007), do not have any inhibitory transmission (Saito et al., 2010), and show other features like insensitivity to tail pinch, kyphotic (hunched posture), omphalocele and cleft palate (Wojcik et al., 2006; Fujii et al., 2007; Saito et al., 2010). The VIAAT KO also shows reduced surface expression of $\mathrm{GABA}_{A} \mathrm{R}$ and GlyR (Wojcik et al., 2006). Similar to GAD67 KO embryos, VIAAT KO embryos have reduced respiratory and gained non-respiratory muscles (Fogarty et al., 2013b). 
The $G_{A B A} R$ and the GlyR mediated inhibitory transmissions are essential for development and maturation of motoneurons (Aguayo et al., 2004). The clustering of the $G A B A_{A} R$ and the GlyR into postsynaptic membrane requires the scaffolding protein gephyrin (SassoePognetto et al., 1995; Feng et al., 1998). Thus, gephyrin KO mice have increased spontaneous hypoglossal motoneurons activity in vitro, reduced respiratory motoneurons survival, and decreased phrenic innervations to diaphragm (Banks et al., 2005). Similar reduced respiratory motoneuron survival, and decreased phrenic innervations to diaphragm are observed in GAD67 KO embryos (Fogarty et al., 2013a). The non-neuronal tissues also require the GABAergic transmission for their normal development and this was experimentally shown by inducing a mutation in the $\beta-3$ unit of $G A B A_{A} R$ that results in the cleft palate (Culiat et al., 1995). Pharmacological blockade and genetic deletion of GAT1 result in neuronal excitability as shown by brain slice studies (Jensen et al., 2003; Semyanov et al., 2003). Similarly, GlyT1 KO mice show suppressed respiratory activity leading to death on the first postnatal day even though the CNS anatomy is normal (Gomeza et al., 2003a). The GlyT2 KO mice show hyperekplexia, spasticity, tremor and finally death in the second postnatal week (Gomeza et al., 2003b; Latal et al., 2010).

\subsection{Aim of this study}

To analyze the role of co-transmission in the respiratory network, co-transmission has been quantified based on the decay kinetics of mIPSCs and co-expression of inhibitory neuronal markers (GlyT2, GAD67, GAD65, and GAT1) in the preBötC glycinergic neurons by Patchclamp recordings and single cell reverse transcription polymerase chain reactions (sc-RTPCR) using GlyT2-EGFP neonatal mice. Furthermore, the role of co-transmission was attempted to study in conditional VIAAT KO (cVIAAT KO) mouse embryos where VIAAT was removed by cre-recombinase action from neurons which express GlyT2 promoter. Since glycinergic neurons in the preBötC receive mixed-mIPSCs and co-express inhibitory neuronal markers, they were further characterized using Patch-clamp and dye filling experiments. 


\section{Materials and methods}

Table 1 Chemicals and reagents

\begin{tabular}{|c|c|}
\hline Reagents/Chemicals & Company/supplier \\
\hline Sodium Chloride $(\mathrm{NaCl})$ & Merck Millipore GmbH \\
\hline Potassium Chloride (KCl) & Merck Millipore GmbH \\
\hline Sodium dihydrogen phosphate $\left(\mathrm{NaH}_{2} \mathrm{PO}_{4}\right)$ & Merck Millipore GmbH \\
\hline Clacium chloride $\left(\mathrm{CaCl}_{2}\right)$ & Sigma-Aldrich AG GmbH \\
\hline Magnesium Chloride $\left(\mathrm{MgCl}_{2}\right)$ & Merck Millipore GmbH \\
\hline Glucose & Merck Millipore GmbH \\
\hline Sodium bi-carbonate $\left(\mathrm{NaHCO}_{3}\right)$ & Sigma-Aldrich AG GmbH \\
\hline $\mathrm{N}-2$-Hydroxyethylpiperazine- N'-2-ethane sulfonic acid (HEPES) & Biomol GmbH \\
\hline Adenosine-tri-phosphate (ATP) & Sigma-Aldrich AG GmbH \\
\hline Ethylene-glycol- tetraacetic-acid (EGTA) & Sigma-Aldrich AG GmbH \\
\hline D-gluconic acid & Sigma-Aldrich AG GmbH \\
\hline Cesium Chloride $(\mathrm{CsCl})$ & Sigma-Aldrich AG GmbH \\
\hline Tetraethylammonium chloride (TEACl) & Sigma-Aldrich AG GmbH \\
\hline Triton $\mathrm{X}-100$ & Sigma-Aldrich AG GmbH \\
\hline Sodium azide & Sigma-Aldrich AG GmbH \\
\hline Paraformaldehyde (PFA) & Sigma-Aldrich AG GmbH \\
\hline Isofluorane & Abbott Deutschland GmbH \\
\hline Methanol & Merck Millipore GmbH \\
\hline Dimethyl sulfoxide (DMSO) & Sigma-Aldrich AG GmbH \\
\hline Tween 20 & Sigma-Aldrich AG GmbH \\
\hline Bovine Serum Albumin (BSA) & SERVA Electrophoresis $\mathrm{GmbH}$ \\
\hline Benzyl benzoate & Sigma-Aldrich AG GmbH \\
\hline Benzyl alcohol & Sigma-Aldrich AG GmbH \\
\hline Sucrose & Carl Roth GmbH \\
\hline
\end{tabular}


Table 2 Reagents used in singel cell reverse transcription PCR

\begin{tabular}{|l|l|l|}
\hline \multicolumn{1}{|c|}{ Reagents/Chemicals } & \multicolumn{1}{|c|}{$\begin{array}{c}\text { Working } \\
\text { Concentration }\end{array}$} & \multicolumn{1}{|c|}{ Company/supplier } \\
\hline Reverse transcriptase buffer & $1 \mu \mathrm{l}$ & Invitrogen, Germany \\
\hline Random hexamer primers & $5 \mu \mathrm{M}$ & Thermo Scientific, Germany \\
\hline Dithiothreitol & $10 \mathrm{mM}$ & Invitrogen, Germany \\
\hline RANaseOUT & $1 \mathrm{U}$ & Invitrogen, Germany \\
\hline Reverse transcriptase Superscript III & $100 \mathrm{U}$ & Invitrogen, Germany \\
\hline Deoxyribonucleosidetriphosphate (dNTP) & $100 \mu \mathrm{M}$ & Thermo Scientific, Germany \\
\hline RedTaq Polymerase & $2.5 \mathrm{U}$ & Sigma-Aldrich AG GmbH \\
\hline
\end{tabular}

Table 3 List of drugs

\begin{tabular}{|l|l|l|l|}
\hline \multicolumn{1}{|c|}{ Name of Drugs } & \multicolumn{1}{|c|}{$\begin{array}{c}\text { Stock } \\
\text { Solvent }\end{array}$} & $\begin{array}{c}\text { Working } \\
\text { Concentration } \\
\text { (in ACSF) }\end{array}$ & \multicolumn{1}{|c|}{ Company } \\
\hline $\begin{array}{l}\text { 6-cyano-7-nitroquinoxaline-2,3-dione } \\
\text { (CNQX) }\end{array}$ & DMSO & $20 \mu \mathrm{M}$ & Tocris (R\&D Systems GmbH) \\
\hline $\begin{array}{l}\text { DL-2-amino-5-phosphonopentanoate } \\
\text { (DL-AP5) }\end{array}$ & $\mathrm{NaOH}$ & $100 \mu \mathrm{M}$ & Tocris (R\&D Systems GmbH) \\
\hline Bicuculline (Bic) & Water & $20 \mu \mathrm{M}$ & Sigma-Aldrich AG GmbH \\
\hline Strychnine (Stry) & Water & $3 \mu \mathrm{M}$ & Sigma-Aldrich AG GmbH \\
\hline Tetradotoxin (TTX) & Acitic acid & $0.5 \mu \mathrm{M}$ & Tocris (R\&D Systems GmbH) \\
\hline Zolpidem & DMSO & $0.5 \mu \mathrm{M}$ & Sigma-Aldrich AG GmbH \\
\hline Sucrose & ACSF & $300 \mathrm{mM}$ & Carl Roth GmbH \\
\hline$\gamma$-amino butyric acid (GABA) & Ringer sol & $10 \mathrm{mM}$ & Sigma-Aldrich AG GmbH \\
\hline Glycine(Gly) & Water & $1 \mathrm{mM}$ & Sigma-Aldrich AG GmbH \\
\hline
\end{tabular}


Table 4 List of primers

\begin{tabular}{|l|l|l|}
\hline Transcript & \multicolumn{1}{|c|}{ Primer for first PCR } & \multicolumn{1}{|c|}{ Primer for second PCR } \\
\hline GAD1 & s: GTGGATGCTGCTTGGGGT & s: GTGGGATTTGAAAACCAGATCAA \\
\hline & as: GAAGAAGTTGACCTTGTCCC (583) & as: TTTTCTCGTCGCTCAGGGC (189) \\
\hline GAD2 & s: GTGGATGCTGCTTGGGGT & s: TACTGGGTTTGAGGCACACATT \\
\hline GAT1 & as: GAAGAAGTTGACCTTGTCCC (583) & as: GCGGCTCATTCTCTCTTCAT (184) \\
\hline & as: GAATTTCTGCATCTGGAAGG & s: TATCATCCTGTTCTTCCGTGGA \\
\hline GlyT2 & s: GCAAAGTCAACATTGAGAATGTG & as: AGGAGTTGATGCAGCAAACGAT (238) \\
\hline & as:AGATACATTTTTATCACGAACATAATC & $\begin{array}{l}\text { as:TCATCATCTCGATGTTTTTTCTGATGCTTCTCAC } \\
\text { (311) }\end{array}$ \\
\hline (625) & s: GATATATCGCTGCGCTGGTCGTC & s: CGTGGGCCGCCCTAGGCACCA \\
\hline & as: CATGGCTGGGGTGTTGAAGGTC (387) & as: CTTAGGGTTCAGGGGGG (238) \\
\hline
\end{tabular}




\subsection{Mouse lines}

Mice handlings and experiments have been carried out according to the guidelines of the "European Parliament and the Council of 22 September 2010" on the protection of animals used for scientific purposes and in accordance with the German Animals Protection Act (Tierschutzgesetz; TierSchG $\S 4$ Abs. 3). The details of animal handling were communicated to the animal welfare official of University Medical Center, Göttingen (Institutional Act number - T11/27). The mice used for experiments were bred and grown either in the animal facility of Institute of Physiology and Pathophysiology, University Medical Center or Max Planck Institute for Experimental Medicine in Göttingen.

\section{GlyT2-EGFP mice}

GlyT2-EGFP (Tg(Slc6a5-EGFP)1Uze) mice that express enhanced green fluorescent Protein (EGFP) under control of neuronal glycine transporter 2 (GlyT2) promoter, were used for identification of glycinergic neurons (Zeilhofer et al., 2010). Electrophysiological experiments and sc-RT-PCR were carried out on slices from neonates (P0-P10) of this mouse line.

\section{Conditional VIAAT knockout mice}

To study the effect of VIAAT removal from glycinergic neurons on overall co-release of GABA and glycine, a conditional VIAAT KO (CVIAAT KO) mouse line was generated by crossing homozygous or heterozygous floxed VIAAT female (contributed by Dr. Sonja Wojcik, MPIEM, Göttingen, unpublished) with BAC-transgenic GlyT2-Cre positive male that were heterozygous for floxed VIAAT allele (Tg(SIc6a5-icre)121Veul) (Ishihara et al., 2010). Since pubs did not survive after birth, a timed mating was carried out to get embryos of particular age (E18.5) for experiments. The detection day of vaginal plug was numbered as E0.5. Offspring with GlyT2-cre and homozygous VIAAT floxed alleles were considered cVIAAT KO, while GlyT2-cre and heterozygous VIAAT floxed allele were considered as heterozygous and remaining embryos were considered as control (Ctrl). Embryos $(\mathrm{N}=369)$ were genotyped to check the distribution of floxed alleles and GlyT2-cre (control-220, heterozygous-83, and KO$66)$. 


\subsection{Slice preparations}

Two kinds of slices containing the preBötC nuclei were cut. Thin slices $(250-300 \mu \mathrm{m})$ contained only a part of the preBötC whereas the rhythmic slice $(650 \mu \mathrm{m})$ contained all or most of the preBötC. The rhythmic slice was required to identify respiratory glycinergic neurons that receive synaptic inputs in-phase with mass activity in the preBötC.

\section{Thin medullary slices}

Neonatal GLYT2-EGFP mice were deeply anesthetized under a fume hood using diethyl-ether and decapitated. The brain was removed from the skull and cooled in ice-cold and carbogen saturated artificial cerebrospinal fluid (ACSF) (in mM; $118 \mathrm{NaCl}, 3 \mathrm{KCl}, 1.5 \mathrm{CaCl}_{2}, 1 \mathrm{MgCl}_{2}, 1$ $\mathrm{NaH}_{2} \mathrm{PO}_{4}, 25 \mathrm{NaHCO}_{3}$, and $30 \mathrm{D}$-glucose). The osmolarity of ACSF was $\sim 310 \mathrm{mOsm} / \mathrm{l}$ and the $\mathrm{pH}$ was adjusted to 7.4 with $1 \mathrm{M} \mathrm{NaOH}$. Brainstem was separated from forebrain and midbrain in carbogen saturated ACSF. An agar block was cut at $20^{\circ}$ angle and glued on a specimen plate. The brainstem was glued on the agar block on its dorsal side so ventral surface of the brainstem was accessible to carbogen saturated ACSF during slicing. The specimen plate with glued brainstem was transferred Microtome (VT1200S, Leica, Bensheim) and coronal slices of different thickness were cut in rostral-caudal direction until hypoglossal and principle nucleus of inferior olive were identified. Since the preBötC starts at this level (Ramirez et al., 1996), 2-3 slices (250-300 $\mu \mathrm{m}$ each) were cut from each brainstem and kept in a custom made chamber containing carbogenated ACSF at room temperature until used for experiments. For embryonic slices, pregnant mice bearing embryos were anesthetized using isofluorane (Abbott Deutschland GmbH \& Co. Wiesbaden, Germany) and killed by cervical dislocation. Uterus was opened after making abdominal cesarean section (CS) and embryos were taken out, cleaned, stimulated by gentle touch on naval remnant and tail pinching to start breathing and placed in warmed area $\left(\sim 30^{\circ} \mathrm{C}\right)$. The embryos, one after another, were anesthetized under ice, decapitated and whole brain was quickly removed from skull. The remaining procedure was the same as of neonatal mice. 


\section{Rhythmic medullary slice}

The procedure of cutting rhythmic slices was same as thin slices except after reaching the level of principle nucleus of inferior olive, instead of 2-3 slices, one thick slice (650 $\mu \mathrm{m})$ was cut that could produce spontaneous rhythmic bursts in $8 \mathrm{mM} \mathrm{K}^{+}$containing ACSF, mimicking respiratory activity (Smith et al., 1991; Ramirez et al., 1996). This was necessary to identify respiratory rhythmic glycinergic neurons; therefore only one slice per neonate or embryo was made.

\subsection{Electrophysiology}

Electrophysiological recordings were made using a Multiclamp 700B amplifier in combination with DigiData (1440) interface and pClamp10 software, (Molecular Devices, Sunnyvale, CA, USA). The electrophysiological recorded signals were filtered at $2-3 \mathrm{kHz}$ and passed through hum-bug eliminator (Quest Scientific, North Vancouver, Canada) to remove $50 \mathrm{~Hz}$ hum. The signals were digitized at $10 \mathrm{kHz}$ before they were saved for analysis.

\section{Whole-cell recording}

The slice was placed in the recording chamber (volume-2ml) which was continuously superfused with bath solution (carbogenated ACSF) at $4 \mathrm{ml} / \mathrm{min}$. The preBötC was identified under epiflourescent microscope under 5X objective. Slices from GLYT2-EGFP mice were illuminated with a $470 \mathrm{~nm}$ emitting photodiode (Rapp OptoElectronic GmbH, Hamburg, Germany) or a monochromator (excitation $467 \mathrm{~nm}$; Polychrome II, TILL Photonics, Gräfelling Germany) fibre-coupled with the upright microscope (Axioscope FS, Zeiss, Oberkochen, Germany) to identify glycinergic neurons. The microscope was equipped with a dichroic mirror $505 \mathrm{~nm}$ and a band pass emission-filter 545/50 nm. The fluorescence-signals were captured using a CCD-camera (Sensicam, PCO, Kehlheim, Germany), controlled by software (Imaging Workbench 6; Indec BioSystems, Santa Clara, USA). To optimise cell visualisation, epifluorescence illumination was combined with transmitted infrared light illumination using a 'gradient contrast' (Dodt \& Zieglgansberger, 1990) and a CCD camera (Vx45 Optronics; Goleta, CA) to display on a TV monitor. 
Patch electrodes were made from borosilicate glass capillaries (OD-1.5mm, ID-0.86mm) (Biomedical Instruments, Zöllnitz, Germany) using horizontal pipette-puller (Zeitz, Munich, Germany). The Patched electrodes were filled with either intracellular solution 1 (in $\mathrm{mM}$; $110 \mathrm{CsCl}, 1.0 \mathrm{CaCl}_{2}, 2 \mathrm{MgCl}_{2}, 4 \mathrm{Na}_{2} \mathrm{ATP}, 10$ EGTA, 10 HEPES, $30 \mathrm{TEACl}$, and $5 \mathrm{QX}-314$ ) to record mIPSCs, or intracellular solution 2 (in $\mathrm{mM}$; $140 \mathrm{KCl}, 1 \mathrm{CaCl}_{2}, 2 \mathrm{MgCl}_{2}, 4 \mathrm{Na}_{2} \mathrm{ATP}, 10$ HEPES, 10 EGTA) to record SIPSCS and eIPSCS, AP and for paired recording, or intracellular solution 3 (in mM; $140 \mathrm{~K}$-gluconic acid, $1 \mathrm{CaCl}_{2}, 2 \mathrm{MgCl}_{2}, 4 \mathrm{Na}_{2} \mathrm{ATP}, 10$ EGTA and 10 HEPES) to characterize the glycinergic neurons. The intracellular solution 3 allowed to differentiate between excitatory and inhibitory inputs at holding potential of $-50 \mathrm{mV}$ (Winter et al., 2009). All intracellular solutions were adjusted for $\mathrm{pH}-7.2$ with $\mathrm{KOH}$. Micropipette microloader (Eppendorf AG, 5242-956-003; Y2333450) was used to fill intracellular solution into patch electrodes.

The patch electrode filled with an intracellular solution displayed 4-6 $\mathrm{M} \Omega$ resistance after placing into the bath solution. Positive pressure $(10-20 \mathrm{mmHg})$ was applied from back side of the patch electrode while brought into bath solution using a micromanipulator -"electrode control system SM5" (Luigs \& Neumann). The positive pressure remains applied throughout the movement of the patch electrode to prevent any debris from clogging the electrode tip until the electrode was reached on the membrane of the identified neuron. The movement of electrode was stopped when an indentation on the cell membrane appeared. The positive pressure was released to form a $G \Omega$ seal between the electrode tip and the neuronal membrane. After few minutes of $\mathrm{G} \Omega$ seal formation, a brief suction (negative pressure) was applied to break the membrane leading to whole-cell configuration. After recording the passive properties like membrane capacitance $\left(C_{m}\right)$ and input resistance $\left(R_{\text {in }}\right)$ of neurons in voltage-clamp and resting membrane potential (RP) in current-clamp at zero holding current, gap-free and step-pulse protocols were applied either in voltage-clamp and/or currentclamp to record membrane currents and membrane potentials respectively.

mIPSCs were recorded in bath solution containing excitatory transmission blockers (CNQX and AP5) and AP blocker (TTX) whereas spontaneous inhibitory postsynaptic currents (sIPSCs) and evoked inhibitory postsynaptic currents (eIPSCs) were recorded only in the presence of CNQX and AP5. Zolpidem (a selective agonist for benzodiazepine site of $G A B A_{A} R$ ) was used to increase the decay time constant of GABAergic mIPSCs (Perrais \& Ropert, 1999) 
which had larger decay time than glycinergic mIPSCs. Pure GABAergic mIPSCs and eIPSCs were recorded in the presence of strychnine in the bath solution, while pure glycinergic mIPSCs and eIPSCs were recorded in the presence of bicuculline in the bath solution. Additionally, glycine for glycinergic elPSCs and GABA for GABAergic elPSCs were applied through another patch electrode using a pressure ejection system (NPI-electronic, Tamm, Germany) that was controlled by pClamp software. The application electrode was placed at a distance of $20-30 \mu \mathrm{m}$ from the recording cell.

\section{Field potential recording}

Field potential recording in the preBötC was made to check the presence of respiratory rhythm in the slice. For this, an electrode $(<1 \mathrm{M} \Omega)$ was filled with ACSF and placed on the surface of the preBötC to record neuronal mass activity. The mass activity was amplified with a custom-built alternating current-amplifier (5000-10000 times), band-pass-filtered (0.25$1.5 \mathrm{kHz}$ ), rectified, and integrated (Paynter filter; time constant, 40-70 ms), digitized (10 $\mathrm{kHz})$, using the pClamp and stored on hard disc.

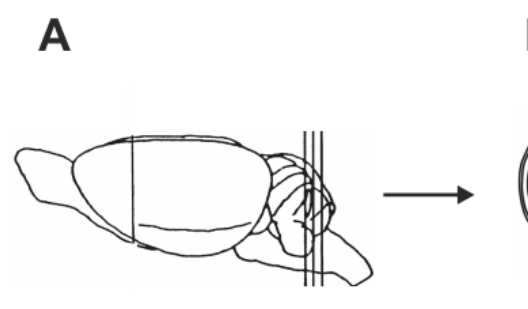

B

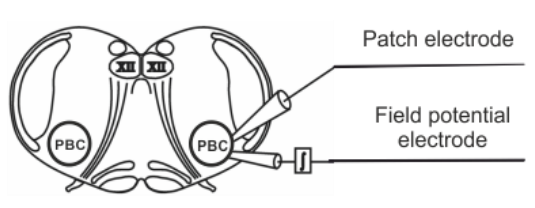

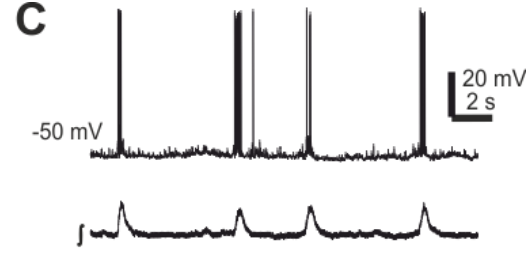

Figure 2.1 Recording from Rhythmic glycinergic neurons.

(A) Drawing shows isolated mouse brain and three lines show the position of coronal brainstem slice. (B) Simultaneous recording through field potential electrode and patch electrode in the preBötC containing coronal brainstem slice. (C) Typical membrane potential trace (upper trace) from a rhythmic glycinergic neuron, which shows the rhythmic bursts in-phase with neuronal population bursts (lower trace) in the preBötC. Figure A was adapted from Scott R. B. et al. 1998 and Figure B was adapted from Tryba A. K. et al. 2004. 


\section{Identification of rhythmic neurons in the preBötC}

Two electrodes were used; one for field potential recording to detect the inspiratory rhythm in the preBötC and another to patch a glycinergic neuron. Once the inspiratory rhythm was detected in the preBötC (Fig. 2.1A, lower trace), another electrode was used to patch and make a whole-cell configuration on a glycinergic neurons. The glycinergic neuron was held in voltage-clamp to observe its membrane currents. If a patched glycinergic neuron received rhythmic inputs during the respiratory rhythm (Fig. 2.1A, upper trace), the neuron was considered to be a respiratory neuron.

\section{Paired recordings}

To study the synaptic strength between glycinergic neurons in the preBötC, pairedrecordings between two glycinergic neurons were made. After making whole cell, cell 1 was voltage-clamped to observe any change in membrane current due to generation of spontaneous action potentials in cell 2 (Fig. 3.18A). Further, to check the opposite synaptic connection between the same pair, the cell 2 was voltage-clamped to observe any change in membrane current due to generation of spontaneous action potentials in cell 1 (Fig. 3.18B). Additionally, the cell 2 was stimulated (in current-clamp) with step-pulse direct current (DC) (110 pA for $500 \mathrm{~ms}$ ) to induce AP (Fig. 3.18C-lower trace) while the cell 1 was held in voltageclamp to observe a corresponding change in IPSCs (Fig. 3.18C-upper trace). Further, to check the opposite synaptic connections between these neurons, the cell 1 was stimulated (in current-clamp) with step-pulse DC (110 pA for $500 \mathrm{~ms}$ ) to induce AP (Fig. 3.18D-upper trace) while cell 2 was held in voltage-clamp to observe changes in IPSCs (Fig. 3.18D-lower trace).

\subsection{Dye filling}

To study the projections of axon and dendrites, different fluorescent dyes were filled through the patch electrodes. Stock solutions of different dyes (Alexa 568, 9.2mM and Alexa $647,8.33 \mathrm{mM}$ ) were made in distilled water, aliquoted and kept at -20 until use. The stocks were diluted in intracellular solution (in $\mathrm{mM} ; 140 \mathrm{KCl}, 1.0 \mathrm{CaCl}_{2}, 2 \mathrm{MgCl}_{2}, 4 \mathrm{Na}_{2} \mathrm{ATP}, 10 \mathrm{EGTA}$ and 10 HEPES), vortexed for $3 \mathrm{~min}$, sonicated for $5 \mathrm{~min}$, to make final working concentrations (0.25mM for Alexa568 and $0.4 \mathrm{mM}$ for Alexa 647). The electrode was back-filled with the working dye solution. The diluted dye solution was not used beyond 2 days. The process of 
patching and making whole-cell configuration for dye filling was the same as described earlier except the applied positive pressure from the back side of electrode was less (5-10 $\mathrm{mmHg}$ ) while approaching the identified cell to minimize the background staining. The cell soma was normally filled in 10-15 minutes but required 15-25 minutes in cell having weak seal between pipette and its membrane. After filling of the cells, the pipette was carefully removed and the slice was fixed in 4\% PFA overnight. The slice was rinsed in PBS (4x5 min), dried, and mounted on Superfrost Plus microscopic slides (Thermo Fischer Scientific GmbH) using Fluorescent Mounting Medium (Dako Industries, Carpinteria, CA). The slices were scanned under LSM 510 to find the filled cells and their projections. The images were processed in Imaris for three dimensional structures and analysis.

\subsection{Immunohistochemistry}

Two different type of immunohistochemistry were carried out; (1) free floating and (2) whole-mount. Free floating immunohistochemistry were performed on thin sections of hindbrain and midbrain whereas whole-mounts immunohistochemistry were performed on complete diaphragms.

\section{Free floating slices}

Brains from CVIAAT KO embryos and their controls were isolated as described in section 2.2 and fixed in PFA (4\%) for 3 days. The whole brain was rinsed in PBS (in $\mathrm{mM} ; 137 \mathrm{NaCl}, 3 \mathrm{KCl}$, 6.5 $\mathrm{Na}_{2} \mathrm{HPO}_{4}$, and $\left.1.5 \mathrm{KH}_{2} \mathrm{PO}_{4}\right)$ and the brainstem was isolated. The brainstem then embedded in agar, and transversely sectioned (rostral to caudal) into $40 \mu \mathrm{m}$ slices with a vibratome (VT1200S; Leica, Bensheim, Germany). The slices were washed (4 x $5 \mathrm{~min}$ ) with PBS to remove excess PFA. These slices were then incubated with blocking buffer (10\% normal donkey serum, 5\% sucrose, $0.4 \%$ Triton $\mathrm{X}-100,0.2 \% \mathrm{Na}$-azide in $1 \times \mathrm{PBS}$ ) for $1 \mathrm{hr}$ at room temperature. The blocking buffer was replaced with staining buffer ( $2 \%$ normal donkey serum, $5 \%$ sucrose, $0.4 \%$ Triton $\mathrm{X}-100,0.2 \%$ Na-azide in $1 \mathrm{x}$ PBS) containing primary antibodies Rabbit anti-VIAAT (1:800; Cat no. 131-003; Synaptic System, Germany) and incubated at $4^{\circ} \mathrm{C}$ for $72 \mathrm{hrs}$. The staining buffer was removed and the slices were washed with $1 \times$ PBS ( 4 x 5 minutes). The washing buffer was replaced with staining buffer containing secondary antibodies Cy3-Donkey-Anti-rabbit-IgG (1:1000; Cat. No. AP182C; Merck 
Millipore, USA) for $2 \mathrm{hrs}$ at room temperature in dark to avoid bleaching of fluorescent dye. Again the slices were washed in $1 \times$ PBS ( $4 \times 5$ minutes) to remove the excess of secondary antibodies. All steps were carried out on shaker $(120 \mathrm{rpm})$. After the last washing, the slices were transferred on Superfrost Plus microscopic slides (Thermo Fischer Scientific GmbH) and allowed to dry for minimum 30 minutes in dark. Finally the slices were mounted under cover slip using Fluorescent Mounting Medium (Dako Industries, Carpinteria, CA). Corners of the cover slip and glass slide were sealed with Nail polish (Young Brilliant Nails, Rival De Loop, Berlin, Germany) to prevent entering of air bubble inside the cover slip and allowed them to dry. The sealed glass slides were kept at $4^{\circ} \mathrm{C}$ until imaging. Minimum two slices from each control embryos were stained for negative controls (Neg Ctrl) where primary antibodies were not used.

\section{Whole mount diaphragm}

To study the effect of cVIAAT KO on phrenic innervations in diaphragm, the embryos were isolated, anesthetized, decapitated, and eviscerated. The diaphragms were carefully isolated from the eviscerated embryos, washed with PBS and then fixed in 4\% PFA for 30 min. Again, the diaphragm was washed overnight in PBS, bleached in Dent's solution (80\% Methanol and 20\% DMSO) for $8 \mathrm{hrs}$ and then rehydrated overnight in PBST (PBS, pH 7.2; 0.1\% Tween-20). After this, the diaphragm was incubated in staining solution (PBS pH 7.2; 1\% bovine serum albumin; $1 \%$ Triton $\mathrm{X}-100)$ containing primary antibodies (1:200; 4H6, Developmental studies, Hybridoma Bank) against Neurofilament (NF) for 3 days and then washed with PBST overnight. Following this, the sample was incubated with secondary antibodies Alexa-488 Donkey anti-mouse (1:2000; Life Technologies $\mathrm{GmbH}$ ) for another 3 days and cleaned in PBST and rehydrated in graded series of methanol solution up to $100 \%$. The sample was washed again in a clearing solution (66\% Benzyl benzoate; 34\% Benzyl alcohol) for proper visualization and then mounted on glass slide under glass cover slip and kept at $4{ }^{\circ} \mathrm{C}$ until imaging. All steps were carried out at $4{ }^{\circ} \mathrm{C}$ unless it is stated. The staining was performed by Dr. Chor Hoon Poh - a collaborator from ENI, Göttingen as described in her PhD thesis (Poh, 2013). 


\subsection{Single cell reverse transcription polymerase chain reaction}

Prior to start single cell reverse transcription polymerase chain reaction (sc-RT-PCR), hand gloves were used to prevent any RNAase contamination that might degrade mRNA. Additionally, the patching electrodes were backed at $300{ }^{\circ} \mathrm{C}$ to make RNAase free. Glycinergic neurons were identified, patched with same pipette solution as of mIPSCs recording, and made into a whole-cell configuration as described in the section 2.3. Cytosol of glycinergic neuron was gradually aspirated into patch electrode ( 1 and 2 in Fig. 3.3A) using a syringe $(10 \mathrm{ml})$. The suction was done very carefully to prevent any extracellular debris or processes of other cell from entering into the suction electrode. The cytosol was ejected from electrode into a PCR tube $(200 \mu \mathrm{L})$ containing master-mix (Table 2: Buffer, DTT, dNTPs, Random hexamer primer, RNAase OUT) by breaking the tip of the electrode and mixed. 0.5 $\mu \mathrm{l}$ reverse transcriptase (Superscript) was immediately added into each PCR tube and further mixed. Following this, the PCR tube was heated at $50{ }^{\circ} \mathrm{C}$ for an hour to reverse transcribe the mRNA into corresponding cDNA. Finally, the PCR tubes were ice cooled for 5-10 minutes and stored at $-80{ }^{\circ} \mathrm{C}$ till used for PCR. Samples for negative control by "mock-aspiration" (an aspiration where patching electrode did not aspirate the cytosol but remaining procedure was same) and positive control using $10 \mathrm{pg}$ of homogenized brain tissue from brainstem were used.

The cDNA was amplified through two steps multiplex nested PCR for different inhibitory neuronal markers (GAD67, GAD65, GAT1 and GlyT2) and $\beta$-actin (as a positive control) (Rahman et al., 2013). In the first-step, cDNA was amplified in the same PCR tube containing cDNA with $1.1 \mathrm{mM}$ of $\mathrm{MgCl}_{2}$, primers $(0.13 \mu \mathrm{M}$ of all primers but $0.1 \mu \mathrm{M}$ of $\beta$-actin primers, see Table 4 for primer sequence), $200 \mu \mathrm{M}$ of each dNTP, 10\% dimethylsulfoxide, and $2.5 \mathrm{U}$ of RedTaq Polymerase (Sigma, Deisenhofen, Germany) in a final volume of $50 \mu \mathrm{l}$ using a protocol $\left(3 \mathrm{~min}\right.$ at $94{ }^{\circ} \mathrm{C}$, followed by 40 cycles, each with $30 \mathrm{~s}$ at $94{ }^{\circ} \mathrm{C}, 30 \mathrm{~s}$ at $49{ }^{\circ} \mathrm{C}$, and 1 $\min$ at $72{ }^{\circ} \mathrm{C}$ ). The final elongation was performed for $10 \mathrm{~min}$ at $72{ }^{\circ} \mathrm{C}$. In a second-step, amplified transcripts from first-step were further amplified using more specific primers (see Table 4) for each inhibitory neuronal marker in separate tubes using the same protocol. $3 \mu \mathrm{l}$ from the first PCR product was added to a new tube containing a reaction mixture which is consist of $1.1 \mathrm{mM} \mathrm{MgCl}$, primers (0.25 $\mu \mathrm{M}$ each; see Table 4 for primer sequence), $200 \mu \mathrm{M}$ of each dNTP, $10 \%$ dimethylsulfoxide, and $2.5 \mathrm{U}$ of RedTaq Polymerase, in a final volume of 
$50 \mu \mathrm{l}$. The final amplified products were separated and visualized on Gel-electrophoresis

(Fig. 3.3B). Positive and negative controls were used to check the experimental success (Rahman et al., 2013).

\subsection{Data analysis}

\section{Electrophysiology}

Electrophysiological data were stored on hard disc of recording PC and then transferred to another PC for analysis. Electrophysiological data were analyzed using Clampfit 10 (Molecular Devices, LLC, Sunnyvale, CA, USA), Igor Pro 6 (WaveMetrics, Inc. Lake Oswego, OR, USA), and MiniAnalysis (Synaptosoft, Chapel Hill, NC). RP and $C_{m}$ were read and noted immediately after making a whole-cell configuration from the Axon software window. $R_{\text {in }}$ was calculated from the change in membrane current with hyperpolarizing step-pulse voltage applied $(-10 \mathrm{mV})$ before each experiment. All postsynaptic currents (sIPSCs, eIPSCs) and APs (frequencies) were analyzed using Clampfit 10.3. mIPSCs were detected with MiniAnlaysis software, visually inspected, and analyzed for amplitude and frequencies. The frequency was calculated as the inverse of interval between two consecutive mIPSCs. However, for decay time constant, only those mIPSCs were analyzed which were clearly separated from each other, therefore, doubled-peak mIPSCs were not considered for analysis of decay time-constant. 


\section{Measurement of contribution of GABA and glycine current to individual mixed mIPSCs}

To determine the contribution of the currents resulting from the activation of glycine or GABA receptors in an individual mixed-mIPSC, individual mIPSC traces were fitted using a piecewise defined exponential product function ( $\Sigma E P F)$.

$$
\boldsymbol{F}(\boldsymbol{t})=\left\{\begin{array}{r}
\boldsymbol{C}, t<\boldsymbol{t}_{0} \\
\boldsymbol{A}_{\boldsymbol{g l y}} \cdot \boldsymbol{I}_{\text {gly }}+\boldsymbol{A}_{G A B A} \cdot \boldsymbol{I}_{G A B A}+\boldsymbol{C}, t \geq \boldsymbol{t}_{0}
\end{array},\right.
$$

with

$$
I_{g l y}=\left(1-e^{\frac{-t-t_{0}}{\tau_{r} g l y}}\right) \cdot\left(e^{\frac{-t-t_{0}}{\tau_{d g l y}}}\right)
$$

and

$$
I_{G A B A}=\left(1-e^{\frac{-t-t_{0}}{\tau_{r} G A B}}\right) \cdot\left(e^{\frac{-t-t_{0}}{\tau_{d_{G A B A}}}}\right)
$$

The parameters for the template mIPSCs, $I_{g l y}$ and $I_{\text {GABA }}$ were derived from averaged pure GABAergic and glycinergic mIPSCs that were recorded from glycinergic neurons (Rahman et al., 2013). $A_{g l y}$ and $A_{G A B A}$ of function 1 ( $\left.\sum E P F\right)$ were used to judge if a mIPSC was a mixed or not. The contribution of glycinergic and GABAergic currents in an individual mIPSC was calculated as described above. mIPSCs were classified as pure glycinergic or GABAergic events if the contribution of the other component did not exceed a threshold value. The mixed-mIPSCs showed different amplitudes and decay kinetics, which were difficult to visually analyze and quantify. Therefore, methods were optimized on simulated traces (Matlab ${ }^{\circledR}$ simulations made by Dr. Tobias Latal) containing a known number of mixedmIPSCs, pure GABAergic mIPSCs and pure glycinergic mIPSCs (Rahman et al 2013). Based on simulated data, a mIPSC was assumed as mono-transmitter event if $\left(A_{g l y} /\left(A_{G A B A}+A_{g l y}\right)\right)<20 \%$ or $\left(A_{G A B A} /\left(A_{G A B A}+A_{g l y}\right)\right)<20 \%$ ( $\left.\sum E P F 20 \%\right)$ (Rahman et al., 2013). The threshold for the decision was also alternatively set to $30 \%$ (LEPF $30 \%)$. 


\section{Single cell reverse transcription polymerase chain reaction}

Electrophoresis gel containing bands of inhibitory markers, following multiplex nested PCR, was transferred to the GelDoc for visualization and imaging. The images were manually analyzed for a band of each inhibitory neuronal marker, positive and negative control. Presence or absence of band for each inhibitory marker and controls were noted down in an Excel spread sheet (Microsoft Office Excel 2007; Microsoft Deutschland, Unterschleißheim, Germany) and analyzed for their summarized result.

\section{VIAAT quantification}

Images were acquired from all immunohistochemically labeled slices, using Laser Scanning Microscope (LSM 510/Axiovert 100 M, Zeiss, Jena, Germany) while parameters (objective, laser intensity, Pinhole, offset, gains) remained same for both cVIAAT KO and their control tissues. Higher magnification (20x) objective was used for better resolution. Since the acquired images were large $(607 \mu \mathrm{m} \times 607 \mu \mathrm{m})$, a region of interest (ROI) was selected from interested areas like hypoglossal nucleus $(173 \mu \mathrm{m} \times 181 \mu \mathrm{m})$, the preBötC $(100 \mu \mathrm{m} \times 100$ $\mu \mathrm{m})$, and hypothalamus $(237 \mu \mathrm{m} \times 237 \mu \mathrm{m})$ for quantification. The size of the ROI was same for both CVIAAT KO and their control slices. The images from Neg Ctrl were opened in ImageJ 1.37a (NIH, USA), histograms were plotted, and mean of maximum intensities was calculated. The CVIAAT KO and their controls images were opened, threshold was set to the mean maximum intensity of negative control staining. Puncta were automatically counted using "analyze particle" option of ImageJ software. The particles on borders were also counted. The summarized results were exported to an Excel spread sheet and analyzed for area fraction. The staining was quantified as the percentage pixel area fraction (stained pixel area/ ROI pixel area).

\section{Diaphragm Analysis}

Complete stained CVIAAT KO diaphragms and their controls were imaged by LSM 510 (Carl Zeiss, Göttingen, Germany) using tile-scanning function. The images were opened in Axiovision software (AxioVs40) and the diameters were measured using length option while area was measured using outline option of Axiovision from both CVIAAT KO diaphragm and their controls. Diameter and area were averaged in an Excel spread sheet. 


\section{Statistical analysis}

All summarized results are written as mean \pm SEM (Standard Error of Mean). Difference in the mean values is compared with two-tailed student's t-Test (Two Samples Assuming equal/unequal Variances) in Excel spread sheet unless it is specified. The statistical significance is represented in bar graphs with asterisk sign (like * if $p<0.05$; ** if $p<0.01$; *** if $p<0.001$ ). Bar graphs were made from mean \pm SEM and transferred into CorelDRAW (Corel $\mathrm{GmbH}$, Munich, Germany) for final alignment of panels. 


\section{Results}

\subsection{GABA and glycine co-transmission in the pre-Bötzinger complex}

Co-transmission of two neurotransmitters are found in different brain regions and hypothesized to be involved in different physiological processes. In the preBötC, glycinergic neurons receive mixed inhibitory synaptic inputs (Winter et al., 2009). Therefore, cotransmission of GABA and glycine to glycinergic neurons in the preBötC of GlyT2-EGFP mice has been studied using electrophysiology and Sc-RT-PCR.

\subsubsection{Glycinergic neurons receive mixed-mIPSCs}

Glycinergic neurons are involved in respiratory rhythm (Winter et al., 2009) and show pacemaker properties (Morgado-Valle et al., 2010). GABAergic and glycinergic IPSCs or mIPSCs are different in their decay kinetics, which becomes more prominent with Zolpidem (Perrais \& Ropert, 1999; Latal et al., 2010). Here, co-transmission of GABA and glycine was studied based on the decay kinetics of mIPSCs, which were recorded from glycinergic neurons in the preBötC, as previously shown in different brain regions (Jonas et al., 1998; Dumoulin et al., 2001; Russier et al., 2002; Nabekura et al., 2004; Awatramani et al., 2005; Dufour et al., 2010). mIPSCs were recorded from a glycinergic neuron after application of blockers against excitatory transmission and AP (Fig. 3.1B). The recorded trace contained three different types of mIPSCs that could be discerned based on their decay kinetics as shown in the enlarged boxes: (1) fast decaying mIPSCs, due to activation of glycinergic receptors only (Fig. 3.1b1), (2) mixed-mIPSCs (have both fast and slow decay component), due to activation of both glycinergic and GABAergic receptors (Fig. 3.1b2), and (3) slow decaying mIPSCs, due to activation of GABAergic receptors only (Fig. 3.1b3). The presence of mixed-mIPSCs confirms that co-release and co-transmission of GABA and glycine to glycinergic neurons is prevalent in the preBötC of GLYT2-EGFP neonatal mice. 
The averaged mixed-mIPSCs to the glycinergic neurons in the preBötC was calculated $32.8 \pm$ $8.3 \%$ by the $\operatorname{EEPF}(30 \%)$ method and $49.0 \pm 12.9 \%$ by the $\Sigma$ EPF (20\%) method (Fig. 3.1D) on control traces. When mixed-mIPSCs were quantified on traces which were subsequently recorded in the presence of bicuculline (Fig. 3.1C), indeed, the averaged mixed-mIPSCs was significantly reduced to $0.90 \pm 1.00 \%$ by the $\operatorname{EEPF}(30 \%)$ and $2.20 \pm 2.50 \%$ by the $\operatorname{EEPF}(20 \%)$ (Fig. 3.1D). One would have not expected any mixed-mIPSC under this condition, therefore, the leftover mixed-mIPSCs were considered as false positive. Finally, the averaged mixedmIPSCs were calculated as the difference between the control and bicuculline conditions which were $31.9 \pm 7.3 \%$ by the $\operatorname{EEPF}(30 \%)$ and $46.8 \pm 10.4 \%$ by the $\Sigma \operatorname{EPF}(20 \%)$ (Fig. 3.1D).

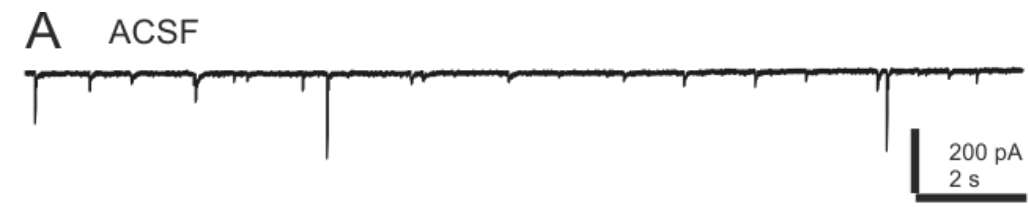

\section{B Control (CNQX + DL-AP5 + TTX)}

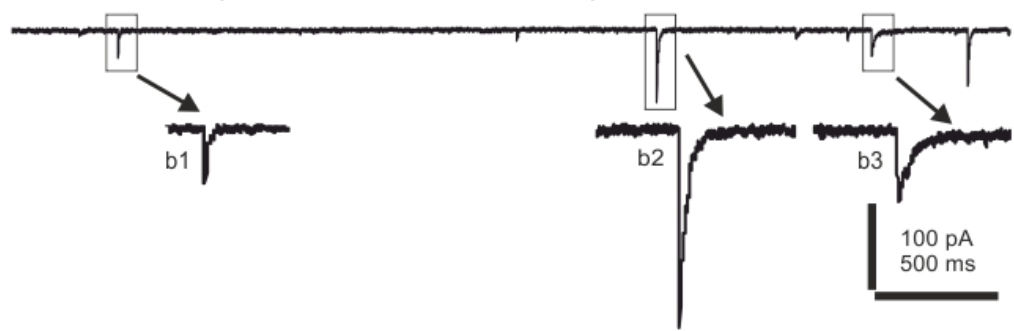

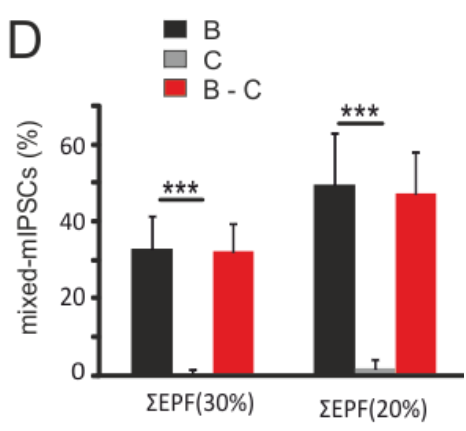

C Control + Bicuculline

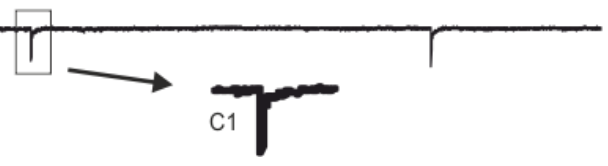

Figure 3.1 Glycinergic neurons in the preBötC receive mixed-mIPSCs.

(A) A representative trace recorded from a glycinergic neuron which receives both EPSCs and IPSCs. (B) A representative trace recorded from the same neuron in the presence of CNQX and DL-AP5, TTX, and Zolpidem shows receiving of mIPSCs. The enlarged mIPSCs clearly looked three types, fast (b1), mixed (b2), and slow (b3). (C) A representative trace recorded from the same cell in the presence of blockers mentioned above and bicuculline, which showed only fast mIPSCs (c1). (D) The summarized results of mixed-mIPSCs quantified by two " $\Sigma E P F "$ methods in control (black bar), Bic (gray bar) conditions and difference (red bar) between these two conditions. 


\subsubsection{Rhythmic glycinergic neurons receive mixed-mIPSCs}

It has been speculated that GABA and glycine co-release is involved in precise regulation of postsynaptic conductance (Jonas et al., 1998). To study the co-release and co-transmission of GABA and glycine to rhythmic glycinergic neurons in the preBötC, the rhythmic glycinergic neurons were identified (Fig. 3.2A) and mIPSCs were recorded (Fig. 3.2B). For rhythmic glycinergic neurons, the averaged mixed-mIPSCs were calculated $21.5 \pm 9.8 \%$ by $\operatorname{EEPF}(30 \%)$ and $39.9 \pm 14.8 \%$ by $\Sigma$ EPF (20\%) (Fig. 3.2D). Again, mixed-mIPSCs were further analyzed on traces which were subsequently recorded in the presence of bicuculline (Fig. 3.2C). Here, the averaged false positive mixed-mIPSCs were $0.5 \pm 1.2 \%$ by $\Sigma \operatorname{EPF}(30 \%)$ and $3.8 \pm 5.1 \%$ by IEPF (20\%) (Fig. 3.2D). Therefore, the averaged mixed-mIPSCs to the rhythmic glycinergic neurons were calculated as the difference between the control and bicuculline conditions which were $21 \pm 8.6 \%$ by the $\Sigma \operatorname{EPF}(30 \%)$ and $36.1 \pm 9.7 \%$ by the $\Sigma \operatorname{EPF}(20 \%)$ (Fig. 3.2D). 


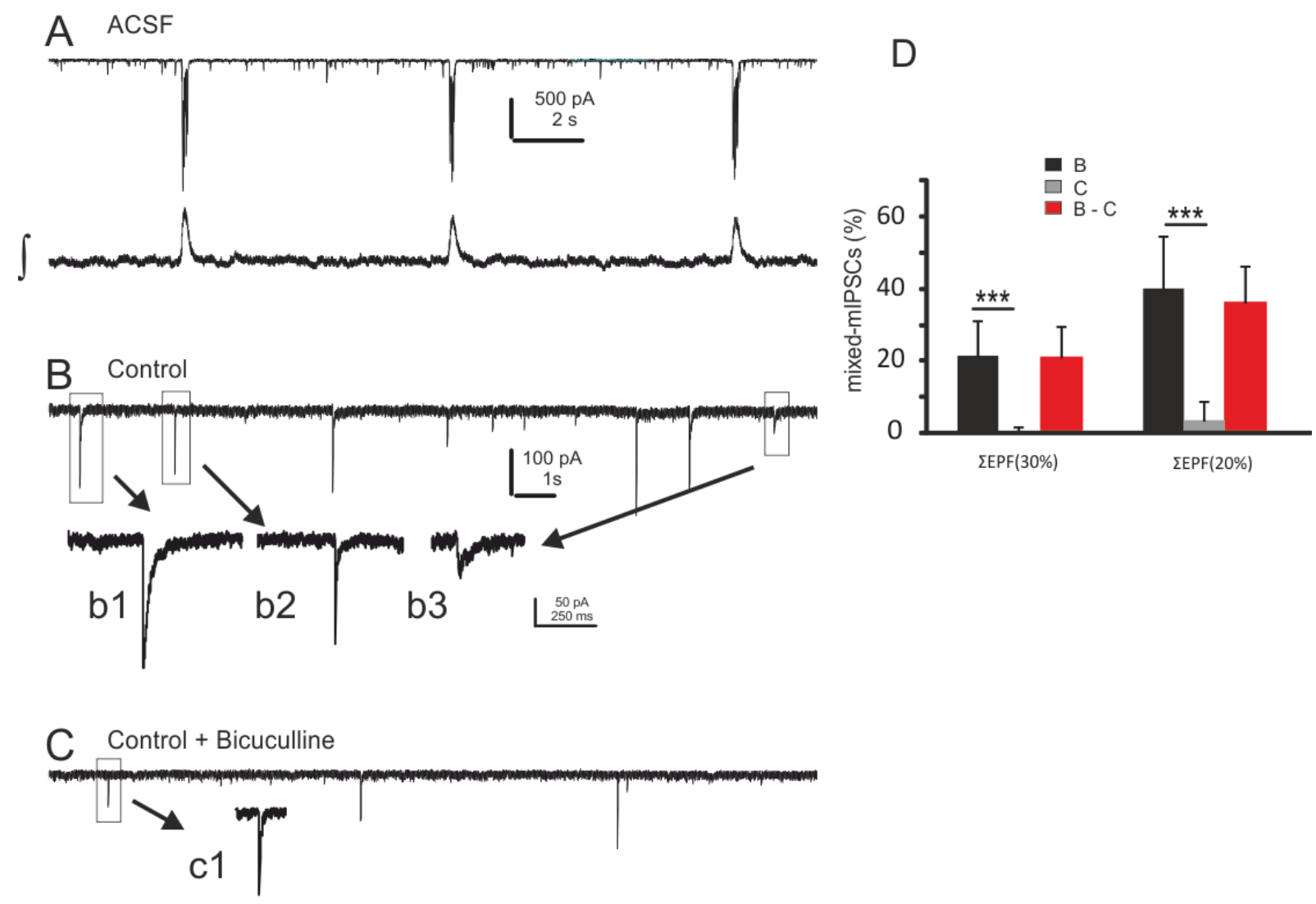

Figure 3.2 Rhythmic glycinergic neurons in the preBötC receive mixed-mIPSCs.

(A) A representative trace recorded from a glycinergic neuron that receives synaptic inputs during respiratory rhythms (upper trace), which confirms that the glycinergic neuron was rhythmic. The trace below recorded from the preBötC shows the integrated mass activities of all neurons. (B) A representative trace recorded from the same glycinergic neuron in the presence of CNQX and DLAP5, TTX, and Zolpidem (control), which showed three different types of mixed-mIPSCs; mixed (b1), fast (b2), and slow (b3)). (C) A representative trace recorded from the neuron in the presence of blockers mentioned above and bicuculline. The trace had mostly fast mIPSCs (Fig. c1, same scale bar as b1-b3). (D) The summarized results of mixed-mIPSCs which were quantified by two " $\Sigma E P F "$ methods in control (black bar), Bic (gray bar) conditions and difference (red bar) between these two conditions. 


\subsubsection{Glycinergic neurons co-express GABAergic and glycinergic neuronal markers}

To use another method for quantification of GABA and glycine co-release in the preBötC, the expression of inhibitory neuronal markers (GAD65, GAD67, GlyT2, and GAT1) in glycinergic neurons were analyzed by single cell reverse transcription polymerase chain reaction (sc-RTPCR). The SC-RT-PCR data from glycinergic neurons showed a heterogeneous expression pattern of inhibitory neuronal markers. Based on this data, glycinergic neurons were classified into six groups (Fig. 3.3B). The group 1 cells expressed GAD and GlyT2, group 2 cells expressed GlyT2 alone, group 3 cells expressed GAD1, GAD2, GAT1, and GlyT2, group 4 cells expressed GAD1 and GAT1, group 5 cells expressed GAD2 and GAT1, and the group 6 cells expressed $\beta$-actin only. The cells from group 1 and group 3 were combined because they expressed both glycine neuronal marker (GlyT2) and at least one GABAergic neuronal marker (GAD65, GAD67, or GAT1) which together represented $46 \%$ of glycinergic neurons. Likewise, the cells from group 4 and group 5 were combined because they expressed only GABA neuronal markers which together represented $18 \%$ of glycinergic neurons cells. The group 2 expressed only GlyT2 marker and represents $2 \%$ of glycinergic neurons. $34 \%$ of glycinergic neurons expressed $\beta$-actin only. Based on summarized results (Fig. 3.3C), $46 \%$ of glycinergic neurons co-expressed both glycinergic and GABAergic neuronal markers, which means that they were definitely capable of filling both GABA and glycine neurotransmitters in the SV and thereby they could co-release too. Furthermore, $18 \%$ glycinergic neurons which expressed only GABAergic markers may also co-release both GABA and glycine. Therefore, in principle, even higher number of glycinergic neurons $(64 \%)$ could release both glycine and GABA. 
A

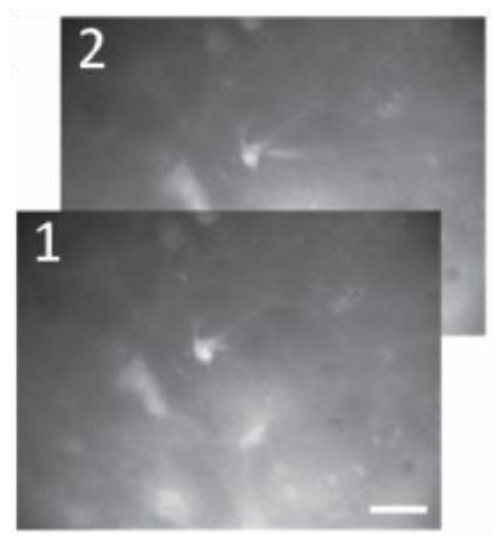

C

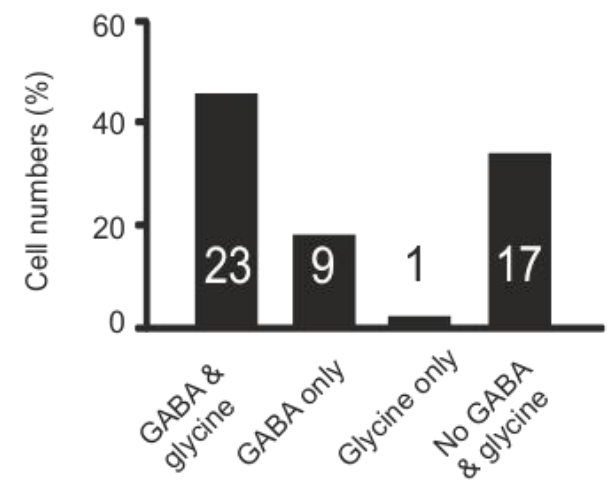

GAD1 GAD2 GAT1 GlyT2 $\beta$-actin Marker

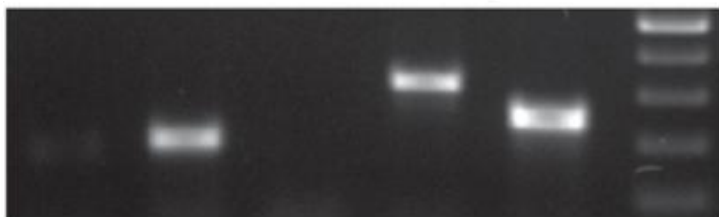

Cell 1

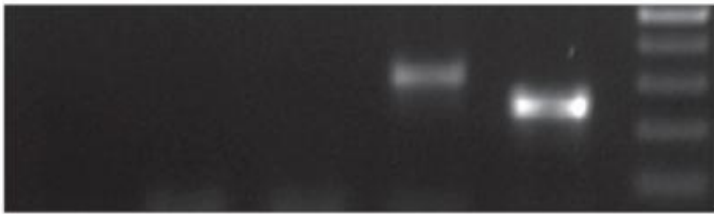

Cell 2

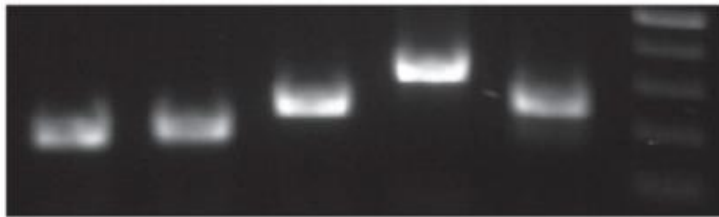

Cell 3

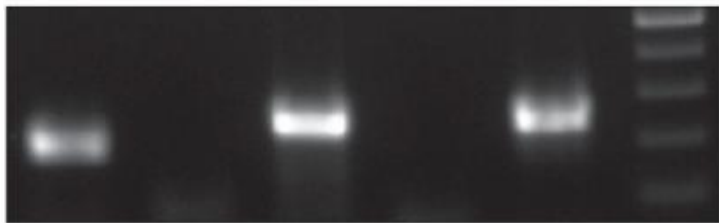

Cell 4

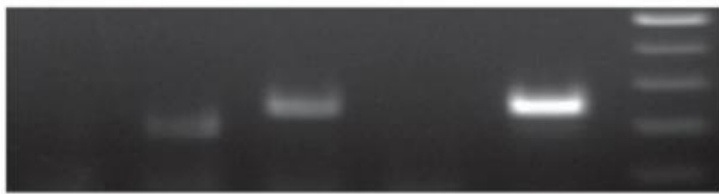

Cell 5

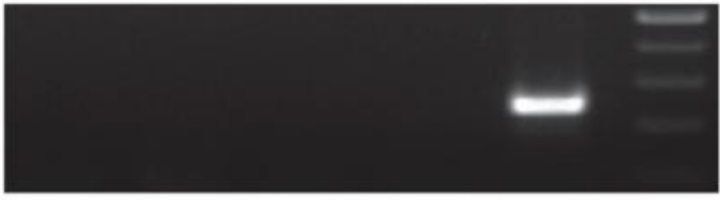

Figure 3.3 Glycinergic neurons show heterogeneous inhibitory molecular markers.

(A) Pictures 1 and 2 from a representative glycinergic neuron show how aspiration was done using a patch electrode. Picture 1 shows the attached-mode of patch electrode while picture 2 shows cytosol aspiration by patch electrode. Scale bar: $20 \mu \mathrm{m}$ (B) Multiplex RT-PCR was preformed to identify the mRNA expression of different inhibitory neuronal markers in glycinergic neurons. Heterogeneous expression of inhibitory molecular markers from 6 different cells is shown. (C) The glycinergic neurons (group 1 to group 6) were assigned into four main groups, based on the expression of inhibitory neuronal markers, are shown. The numbers inside bars represent the neurons analyzed. 


\subsection{Conditional knockout of the vesicular inhibitory amino acid transporter}

The vesicular inhibitory amino acid transporter (VIAAT) is the only vesicular transporter responsible for filling of both GABA and glycine in synaptic vesicle, therefore, it is expressed by both glycinergic and GABAergic neurons (Mclntire et al., 1997; Sagne et al., 1997; Chaudhry et al., 1998). As shown before, glycinergic neurons in the preBötC receive mixed synaptic inputs from neurons which could be either GABAergic or glycinergic or both (Rahman et al., 2013). The sc-RT-PCR results showed that $64 \%$ glycinergic neurons in the preBötC express markers for GABAergic neurons (Rahman et al., 2013). These results have strongly supported the involvement of glycinergic neurons in co-transmission of GABA and glycine in the preBötC. To delineate the detailed role of glycinergic neurons in cotransmission in the respiratory network, a cVIAAT KO mouse line was generated and studied.

\subsubsection{Conditional VIAAT KO embryos show lethal phenotype}

All VIAAT KO embryos do not breathe and have omphalocele, cleft palate, and die after birth (Wojcik et al., 2006; Fujii et al., 2007; Saito et al., 2010). The cVIAAT KO pubs did not survive in any litter; therefore, the cVIAAT KO embryos were studied. The cVIAAT KO embryos showed striking anatomical features like omphalocele ( $N=54 / 54$; arrow in Fig. 3.4A), kyphotic $(N=54 / 54)$, cleft-palate $(N=23 / 23$; arrow in Fig. $3.4 C)$, immobility $(N=54 / 54)$, and irresponsiveness to tail pinch $(N=54 / 54)$, while their controls were normal. Whole body plethysmography of cVIAAT KO embryos showed complete loss of breathing (Fig. 3.4B; $\mathrm{N}=9 / 9)$, while their controls breathed normally ( $0.8 \pm 0.9 \mathrm{~Hz}$; Fig. 3.4B). Since the cVIAAT KO embryos did not breathe, they turned cyanotic while their controls usually remained pink. To check the possibility of initial breathing between CS and plethysmography measurement, their lungs were tested for buoyancy in PBS. While the control lungs $(N=7 / 7)$ were floated, the cVIAAT KO lungs sank in PBS (N=9/9; Fig. 3.4D), which further confirmed the complete absence of breathing in cVIAAT KO embryos. The cVIAAT KO embryos were smaller compared to their controls, so their weights were measured. The averaged weight of cVIAAT KO embryos (1.17 $\pm 0.02 \mathrm{~g} ; \mathrm{N}=28)$ was significantly lower compared to their controls $(1.27 \pm$ 0.02 g; N=94; Fig. 3.4E). 
A

$\mathrm{KO}$

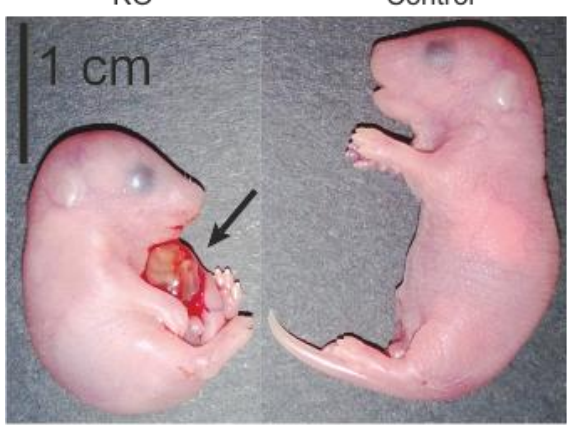

B
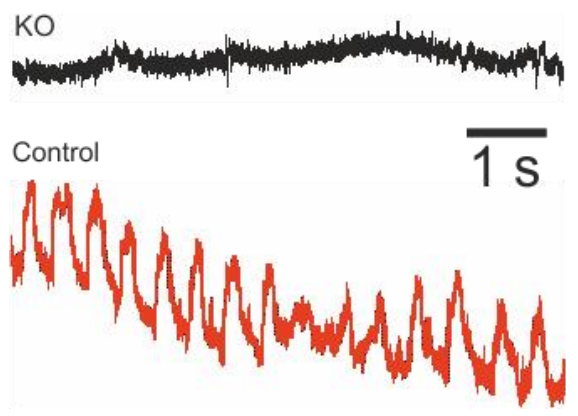

C

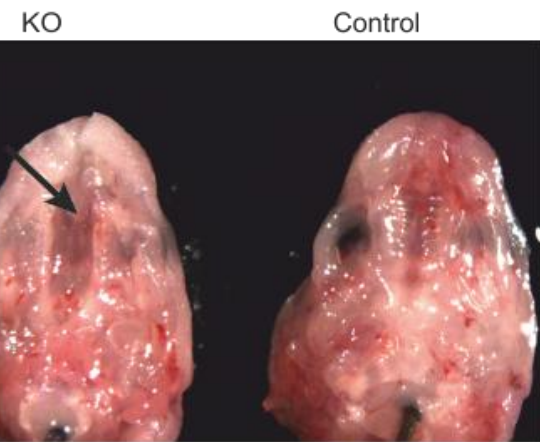

D

$\mathrm{KO}$

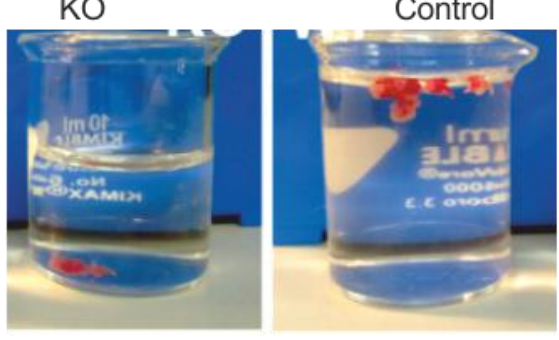

E

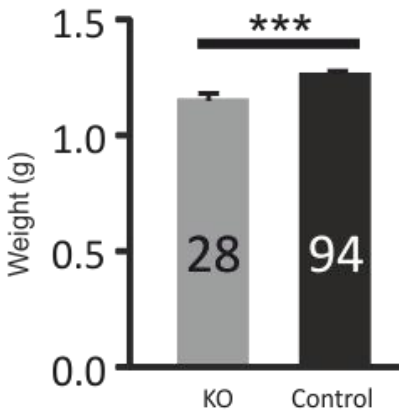

Figure 3.4 cVIAAT KO embryos showed lethal phenotype.

(A) The CVIAAT KO embryos were smaller, kyphotic, and showed omphalocele (see arrow) while their controls (right) were normal. (B) The representative plethysmography trace recorded from a cVIAAT KO embryo did not show any breathing activity while their controls showed normal breathing (red). (C) The palate of cVIAAT KO embryos were not closed (cleft palate, see arrow); whereas it was closed in control embryos. (D) The cVIAAT KO lungs sank while their control lungs floated in PBS. (E) The averaged weight of cVIAAT KO embryos and their controls are shown. Numbers inside bars represent number of embryos weighted for analysis. 


\section{Conditional VIAAT KO embryos are hyperglycemic}

The cre-recombinase (GlyT2-cre) used for selective removal of VIAAT from glycinergic neurons in cVIAAT KO embryos was driven by GlyT2 promoter, which is also expressed in the pancreas (Gammelsaeter et al., 2004). Interestingly, VIAAT is also expressed on vesicle like structure present in both $\alpha$ - and $\beta$-cells of the pancreas (Gammelsaeter et al., 2004). Furthermore, GABA signaling in the pancreas is involved in insulin secretion, which controls plasma glucose level (Taneera et al., 2012). To check a possible role of GlyT2-cre in the pancreas and thereby on glucose level, the plasma glucose level of the cVIAAT KO embryos and their controls was measured. The mean plasma glucose level of cVIAAT KO embryos (5.00 $\pm 0.35 \mathrm{mM} ; \mathrm{N}=12$ ) was significantly higher than the mean plasma glucose level of their controls $(1.83 \pm 0.14 \mathrm{mM} ; \mathrm{N}=51$; Fig. $3.5 \mathrm{~A})$. Mothers of these embryos had also high plasma glucose levels (6.70 $\pm 0.57 \mathrm{mM} ; \mathrm{N}=10)$ as already previously shown (Mogami et al., 2010).

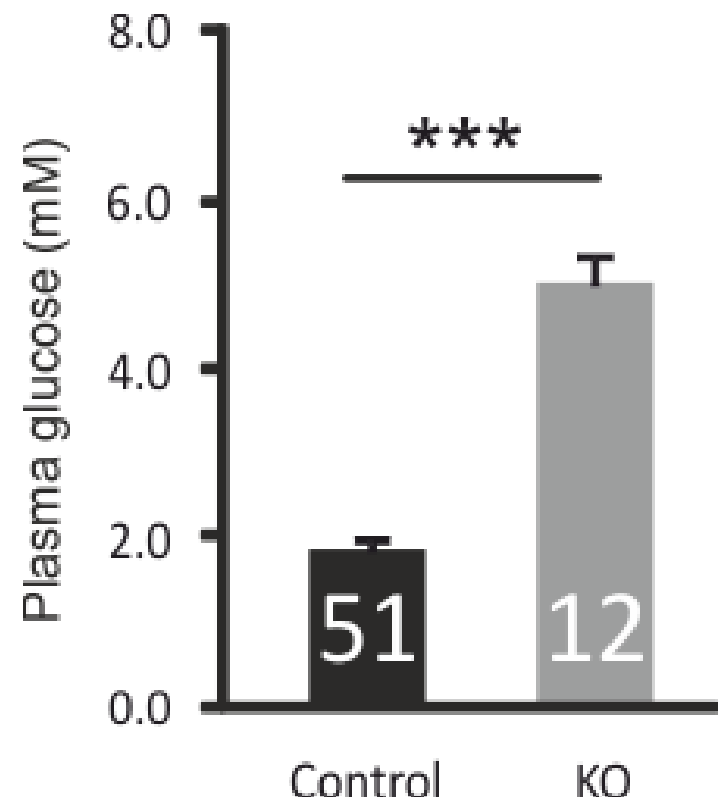

Figure 3.5 cVIAAT embryos were hyperglycemic.

The average plasma glucose level of cVIAAT KO embryos was significantly higher compared to their controls. Numbers inside bar represents number of embryos used for analysis. 


\subsubsection{Respiratory rhythmic activities are present in the cVIAAT KO preBötC}

To study the respiratory network function, the field potential recordings were made in the preBötC of cVIAAT KO rhythmic slices and their controls. The network activity in the cVIAAT KO preBötC $(\mathrm{N}=4 / 6)$ and their controls $(\mathrm{N}=6 / 6)$ were present though it was irregular as reported in newborn mice (Gomeza et al., 2003a). Since, chloride mediated synaptic transmission is inhibitory at birth and blockers of inhibitory transmission changes respiratory pattern, strychnine and bicuculline were applied to the cVIAAT KO rhythmic slices and their controls to observe the change in the respiratory network activity (Onimaru et al., 1990; Gomeza et al., 2003a). The preBötC network activity was increased in control slices (Fig. 3.6A) while it remained unchanged in cVIAAT KO ones (Fig. 3.6B). 

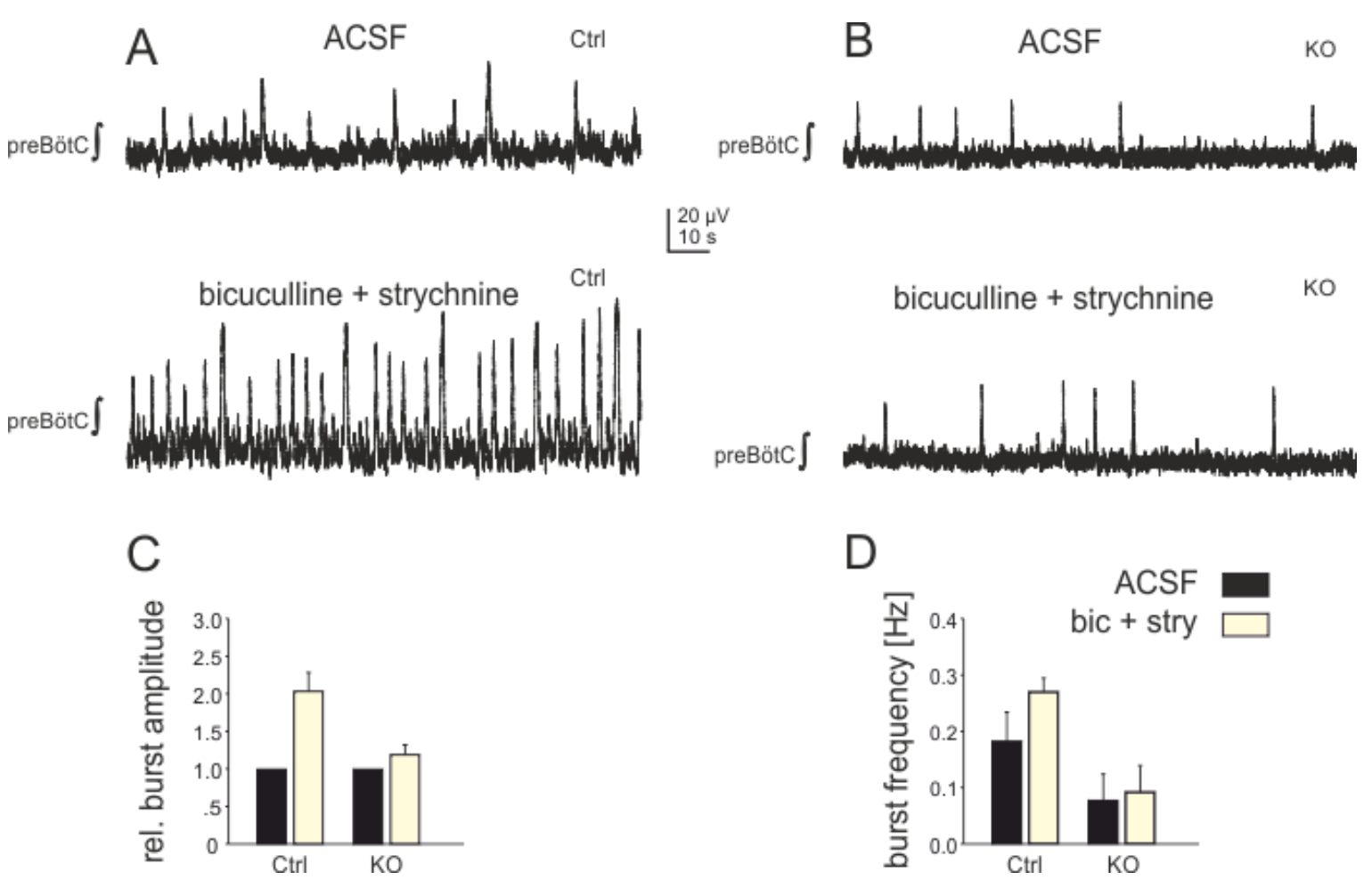

Figure 3.6 Respiratory rhythmic activity in the CVIAAT-KO slices and their control.

(A) A representative field potential recording from the control preBötC in ACSF (upper trace) and in $\mathrm{ACSF}+20 \mu \mathrm{M}$ Bic $+10 \mu \mathrm{M}$ Stry (lower trace) are shown. (B) A representative field potential recording from the cVIAAT KO preBötC in ACSF (upper trace) and in ACSF $+20 \mu \mathrm{M}$ Bic $+10 \mu \mathrm{M}$ Stry (lower trace) are shown. (C) The relative burst amplitudes from cVIAAT KO and their controls in both conditions are summarized. (D) The averaged burst frequencies from cVIAAT KO and their controls in both conditions are summarized. These traces were recorded by Dr. Christian Schnell, CNMPB.

The amplitudes of spontaneous rhythmic bursts recorded in ACSF from both cVIAAT KO preBötC as well as their controls were set as the base line for each genotype separately for relative amplitude calculation. The averaged relative amplitude of spontaneous rhythmic bursts in the presence of Bic and Stry was slightly higher control preBötC $(2.03 \pm 0.25 ; N=5)$ compared to their cVIAAT KO $(1.19 \pm 0.13 ; \mathrm{N}=6$; Fig. $3.6 \mathrm{C})$. The averaged frequencies of spontaneous rhythmic bursts in control preBötC was increased $(0.18 \pm 0.05 \mathrm{~Hz}$ to $0.27 \pm 0.02$ $\mathrm{Hz} ; \mathrm{N}=5)$ while it remained unchanged in the cVIAAT KO $(0.07 \pm 0.04 \mathrm{~Hz}$ vs $0.09 \pm 0.04 \mathrm{~Hz}$; $\mathrm{N}=6$ ) with Bic and Stry application in bath solution. 


\subsubsection{VIAAT expression is reduced in medulla but not in hypothalamus}

\section{VIAAT staining in medulla}

Glycinergic neurons, which express GlyT2 promoter, are abundantly present in brainstem and spinal cord (Zafra et al., 1995).The preBötC generates respiratory rhythm while hypoglossal motoneurons help in respiratory rhythm by tongue movement during deglutition and vocalization; both are located in the hindbrain (Lowe, 1980). The preBötC and hypoglossal nucleus are required for maintain normal respiratory rhythm. Hypoglossal nucleus expresses highest GlyR density compare to any area in CNS, therefore, it is considered as a good model to study inhibitory inputs (White et al., 1990; Singer \& Berger, 2000; Hernandes \& Troncone, 2009). In this study, the hypoglossal nucleus was identified, based on its previously shown anatomical location and through Choline Acetyl Transferase (ChAT) staining in slices from cVIAAT KO embryos and their controls (Fig. 3.7A) (Hulsmann et al., 2000). The VIAAT staining was prominent in control slices (Fig. 3.7B and 3.7C) while drastically reduced in cVIAAT KO slices (Fig. 3.7D). The histogram was plotted from cVIAAT $\mathrm{KO}$ and their controls that showed a clear difference in stained pixel intensity distribution (Fig. 3.7E). The average stained pixel area of the control hypoglossal $(81.48 \pm 2.00 \%$; $=6$ embryos) was significantly higher compared to the average stained pixel areas of the CVIAAT KO hypoglossal ( $4.35 \pm 1.10 \%$; $N=6$ embryos; Fig. 3.7F). 

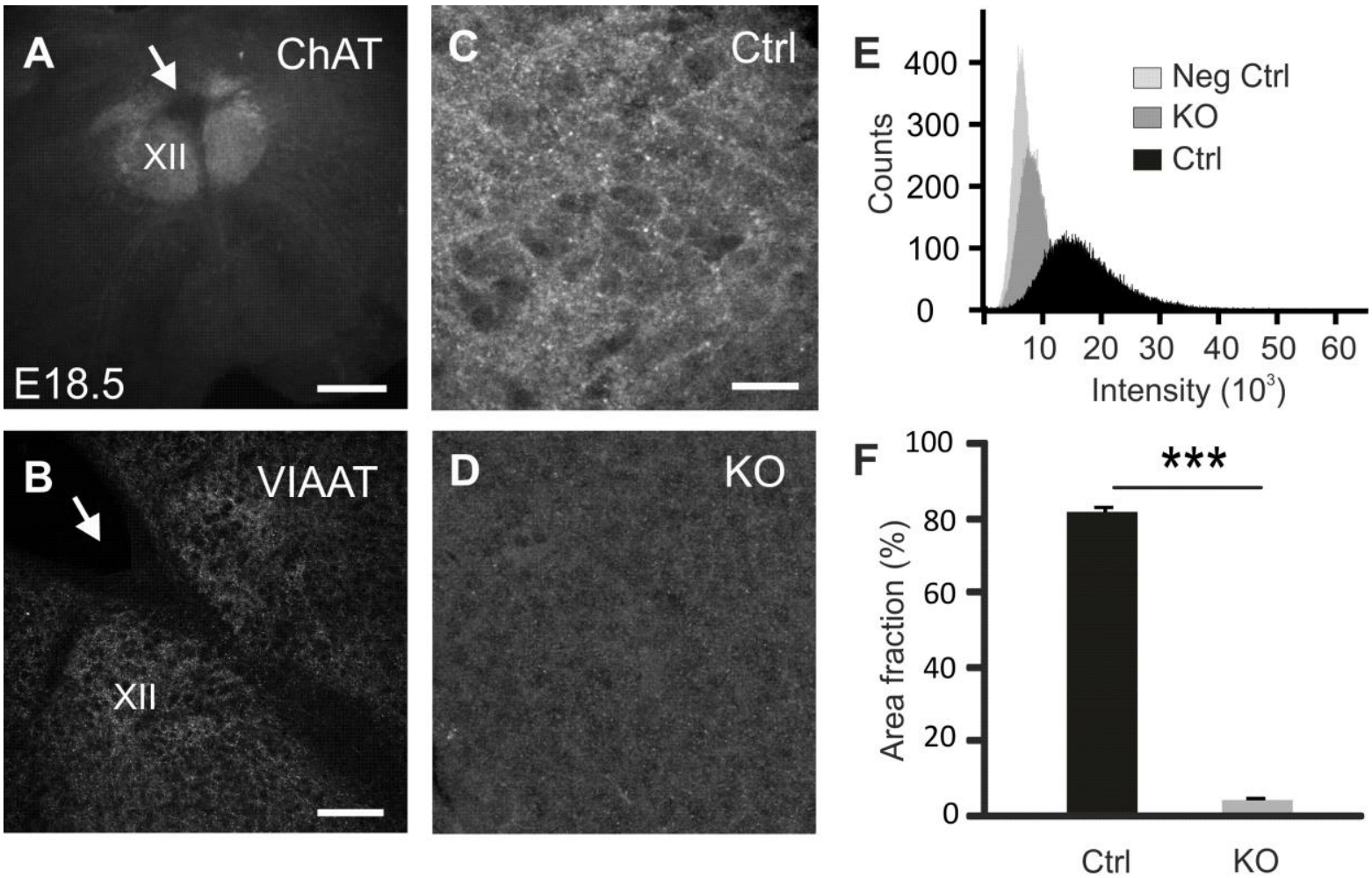

Figure 3.7 VIAAT expression was dramatically reduced in cVIAAT KO hypoglossal nucleus.

(A) Hypoglossal nucleus (XII cranial nerve) of control (Ctrl) embryos was identified via ChAT staining, scale bar: $300 \mu \mathrm{m}$. (B) The control hypoglossal nucleus is prominently stained with VIAAT antibodies, scale bar: $100 \mu \mathrm{m}$. (C) A control hypoglossal ROI showed strong VIAAT staining. (D) In contrast, a CVIAAT KO hypoglossal ROI showed a very weak VIAAT staining. In Fig. C-D, scale bar: $30 \mu \mathrm{m}$. (E) Histogram of negative control, cVIAAT KO, and control ROI showed difference in the distribution of VIAAT stained pixel intensity. (F) The averaged stained pixel area (\%) in cVIAAT KO hypoglossal nucleus was significantly reduced compared to their controls. 
Similar to hypoglossal nucleus, VIAAT staining was stronger in the control preBötC (Fig. 3.8A) compared to CVIAAT KO sample (Fig. 3.8B). The histogram showed a clear difference in the pixel intensity distribution between cVIAAT KO preBötC and their control (Fig. 3.8C). The average stained pixel area in control preBötC (75.72 $\pm 2.19 \%$; $=3$ embryos) was significantly higher compared to the cVIAAT KO preBötC (6.63 $\pm 0.83 \%$; N=3 embryos; Fig. 3.8D;).
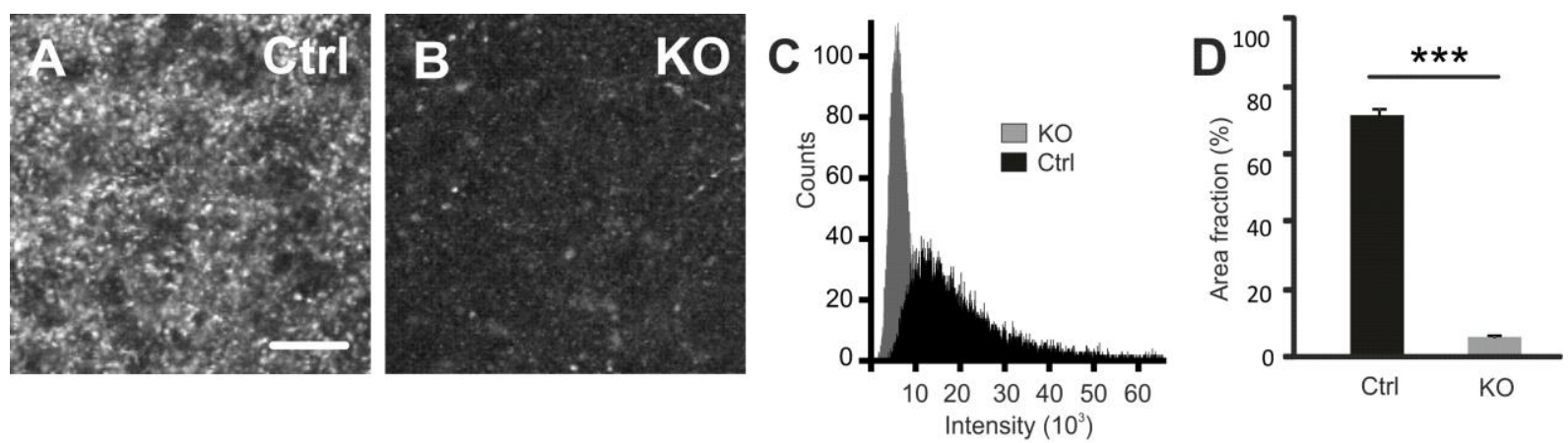

Figure 3.8 VIAAT expression in the CVIAAT KO preBötC was reduced.

(A) A representative control preBötC ROI showed prominent staining with VIAAT antibodies. (B) A representative CVIAAT KO preBötC ROI showed highly reduced VIAAT staining. The scale bar is same for $A$ and B: $20 \mu \mathrm{m}$ (C) Histogram of control and cVIAAT KO preBötC showed a clear difference in VIAAT stained pixel intensity. (D) The average stained pixel area (\%) in CVIAAT KO preBötC was significantly reduced compared to their controls ( $\mathrm{N}=3$ embryos each). 


\section{VIAAT staining in hypothalamus}

Synaptic inhibition is primarily mediated by GABAergic transmission in hypothalamus which lacks glycinergic marker GlyT2 (Decavel \& Van den Pol, 1990; Zafra et al., 1995). Since VIAAT fills both GABA and glycine into SV and VIAAT was removed from those neurons which expressed GlyT2 promoter in cVIAAT KO embryos, the VIAAT expression should not be reduced in hypothalamus (Sagne et al., 1997). To test this, quantification of VIAAT staining were made in CVIAAT KO hypothalamus and their controls. As expected, VIAAT staining did not change between control preBötC (Fig. 3.9 A) and cVIAAT KO hypothalamus (Fig. 3.9B). The histogram also did not show any difference in pixel intensity distribution between cVIAAT KO and control preBötC (Fig. 3.9C). The average stained pixel area in control hypothalamus ( $83.47 \pm 0.85 \%$; $N=4$ embryos) was similar to the average stained pixel area in the cVIAAT KO hypothalamus ( $82.41 \pm 1.10 \%$; N= 4 embryos; Fig. 3.9D).
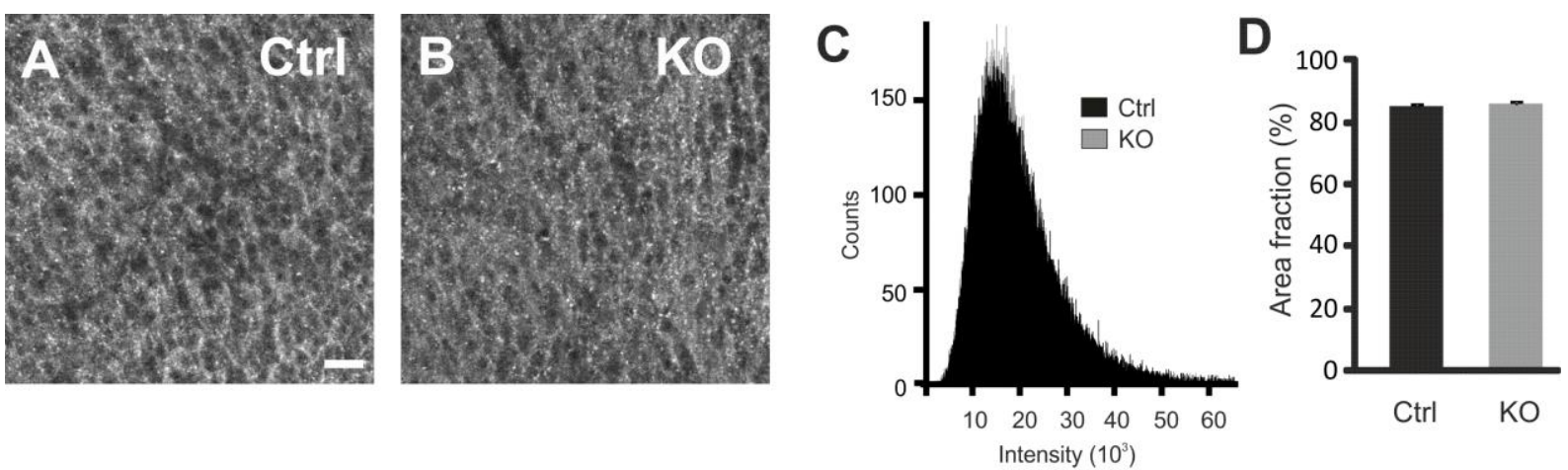

Figure 3.9 VIAAT expression in CVIAAT KO hypothalamus was not reduced.

(A) A representative stained control hypothalamus $\mathrm{ROI}$ showed prominent staining with VIAAT antibodies (B) A representative stained CVIAAT KO hypothalamus ROI also showed similar VIAAT staining. Scale bar is same in A and B: $20 \mu \mathrm{m}$. (C) Histogram of control and cVIAAT KO hypothalamus ROI showed complete overlap in stained pixel intensity distribution (D) The average stained pixel area (\%) in cVIAAT KO hypothalamus was similar to their controls. ( $\mathrm{N}=4$ embryos each). 


\subsubsection{Passive and active properties of hypoglossal motor neurons}

\section{Passive properties}

The passive properties ( $R_{\text {in }}$ and $C_{m}$ ) of hypoglossal motoneurons from both cVIAAT KO and their controls embryos were measured to compare if these properties were changed in cVIAAT KO embryos. The average $R_{\text {in }}$ of cVIAAT KO hypoglossal motoneurons $(0.42 \pm 0.11$ $\mathrm{G} \Omega ; \mathrm{N}=31)$ did not change ( $\mathrm{p}$-values $=0.5)$ compared to their controls $(0.34 \pm 0.05 \mathrm{G} \Omega ; \mathrm{N}=38$ ) (Fig. 3.10A). The average $C_{m}$ of cVIAAT KO hypoglossal motoneurons (44.55 \pm 3.22 pF; N=21) was significantly reduced compared to their control (54.87 $\pm 3.37 \mathrm{pF} ; \mathrm{N}=28 ;$ Fig. 3.10B). The RP depends on different ionic concentration including chloride ions across the cell membrane. The cVIAAT KO mice showed disturbed inhibitory transmission, which is mediated through chloride ions, that might have affected the RP of hypoglossal motoneurons. To test this hypothesis, the RP of cVIAAT KO hypoglossal motoneurons and their controls were measured. The average RP of CVIAAT KO hypoglossal motoneurons ($57.57 \pm 1.98 \mathrm{mV} ; \mathrm{N}=35$ ) was significantly depolarized compared to their control hypoglossal motoneurons (-64.34 $\pm 1.28 \mathrm{mV} ; \mathrm{N}=41$; Fig. 3.10C).

A

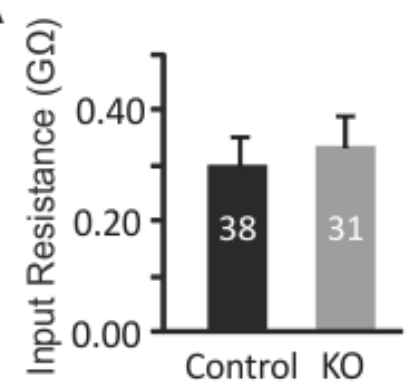

B

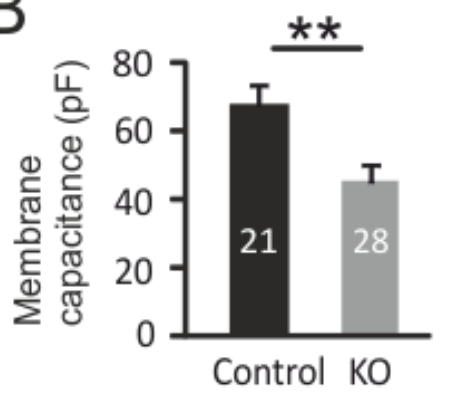

C

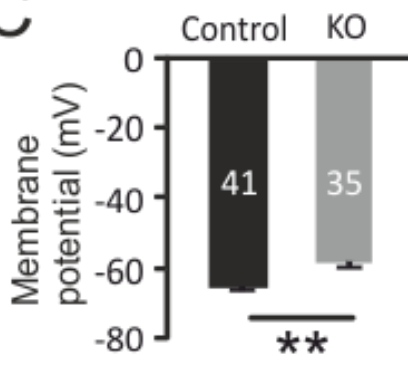

Figure 3.10 Passive properties of hypoglossal motoneurons.

(A) The average $R_{\text {in }}$ of CVIAAT KO hypoglossal motoneurons and their control were the same. (B) The average $C_{m}$ of cVIAAT KO hypoglossal motoneurons was significantly reduced compared to their controls. (C) The average RP of CVIAAT KO hypoglossal motoneurons was significantly depolarized compared to their controls. Numbers inside the bar are analyzed neurons. 


\section{Active properties}

Although cVIAAT KO embryos did not survive after birth, their hypoglossal motoneurons were appeared as healthy as control hypoglossal motoneurons. To confirm this, both cVIAAT KO hypoglossal motoneurons and their controls were depolarized with DC injections. All cVIAAT KO hypoglossal motoneurons as well as their controls showed APs with step-pulses DC injection. The firing pattern of APs in both CVIAAT KO and control hypoglossal motoneurons were similar (Fig. 3.11A). The average APs frequency in cVIAAT hypoglossal motoneurons did not differ from their control with $40 \mathrm{pA}(17 \pm 1.85 \mathrm{~Hz}$ vs $15.84 \pm 1.42 \mathrm{~Hz})$, $60 \mathrm{pA}(19.40 \pm 2.27 \mathrm{~Hz}$ vs $19 \pm 1.57 \mathrm{~Hz})$, and $80 \mathrm{pA}(22.40 \pm 2.40 \mathrm{~Hz}$ vs $20.37 \pm 1.72 \mathrm{~Hz})$ steppulse DC injection (Fig. 3.11B).
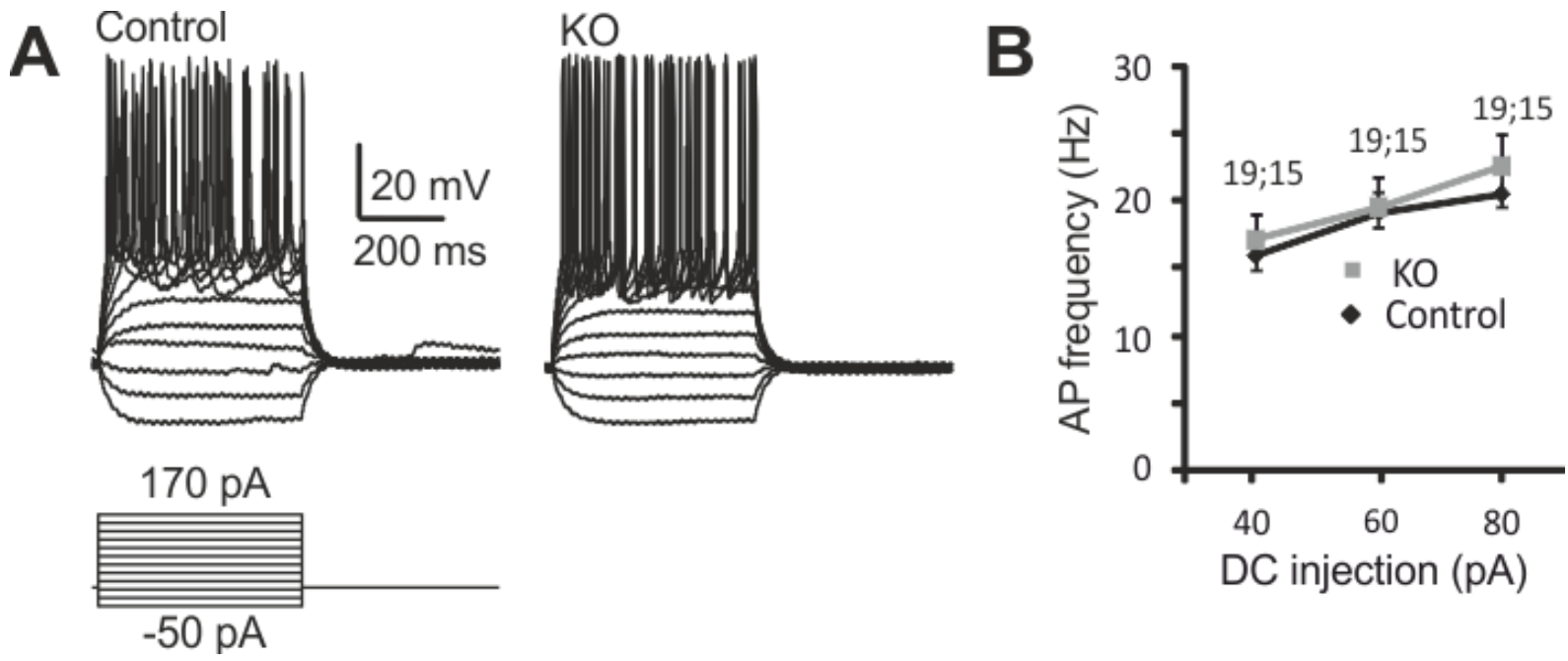

Figure 3.11 cVIAAT KO hypoglossal motoneurons and their controls produced APs.

(A) Representative membrane potential traces recorded from a cVIAAT KO hypoglossal motoneuron and its control showed similar APs pattern with DC injections (below Step-pulse protocol). (B) The average frequencies of CVIAAT KO hypoglossal motoneurons did not change compared to their control with $40 \mathrm{pA}, 60 \mathrm{pA}$ and $80 \mathrm{pA}$ biased DC injection. The numbers above the average values represent the neurons from control and $\mathrm{KO}$ embryos studied respectively. 


\subsubsection{Hypoglossal motoneurons in cVIAAT KO embryos lack inhibitory transmission}

IPSCs in spinal cord of constitutional VIAAT KO embryos are either absent or highly reduced (Wojcik et al., 2006; Saito et al., 2010). The VIAAT expression was significantly reduced in hypoglossal nucleus and the preBötC of cVIAAT KO embryos as shown in immunohistochemical staining. Therefore, to check a possible change in the inhibitory transmission, sIPSCs were also recorded from cVIAAT KO hypoglossal motoneurons and their controls. cVIAAT KO hypoglossal motoneurons did not receive any sIPSC (Fig. 3.12A, lower trace), while, control hypoglossal motoneurons received sIPSCs (Fig. 3.12A, upper trace) with or without sucrose application (300 mM, 10s) (Rosenmund \& Stevens, 1996). The difference between the average amplitude of sIPSCs before (70.05 $\pm 16.42 \mathrm{pA} ; \mathrm{N}=13)$ and after $(75.80 \pm$ 10.10 pA; N=13; Fig. 3.12B) sucrose application remained non-significant ( $p$-values $=0.76$ ) in control hypoglossal motoneurons. Similarly the difference between the average frequencies of sIPSCs before $(4.28 \pm 1.73 \mathrm{~Hz} ; \mathrm{N}=13)$ and after $(4.66 \pm 1.39 \mathrm{~Hz} ; \mathrm{N}=13$; Fig. 3.12C) sucrose application also remained non-significant $(p$-values $=0.80)$ in control hypoglossal motoneurons. The sIPSC in CVIAAT KO hypoglossal motoneurons did not increase with sucrose application. 
A

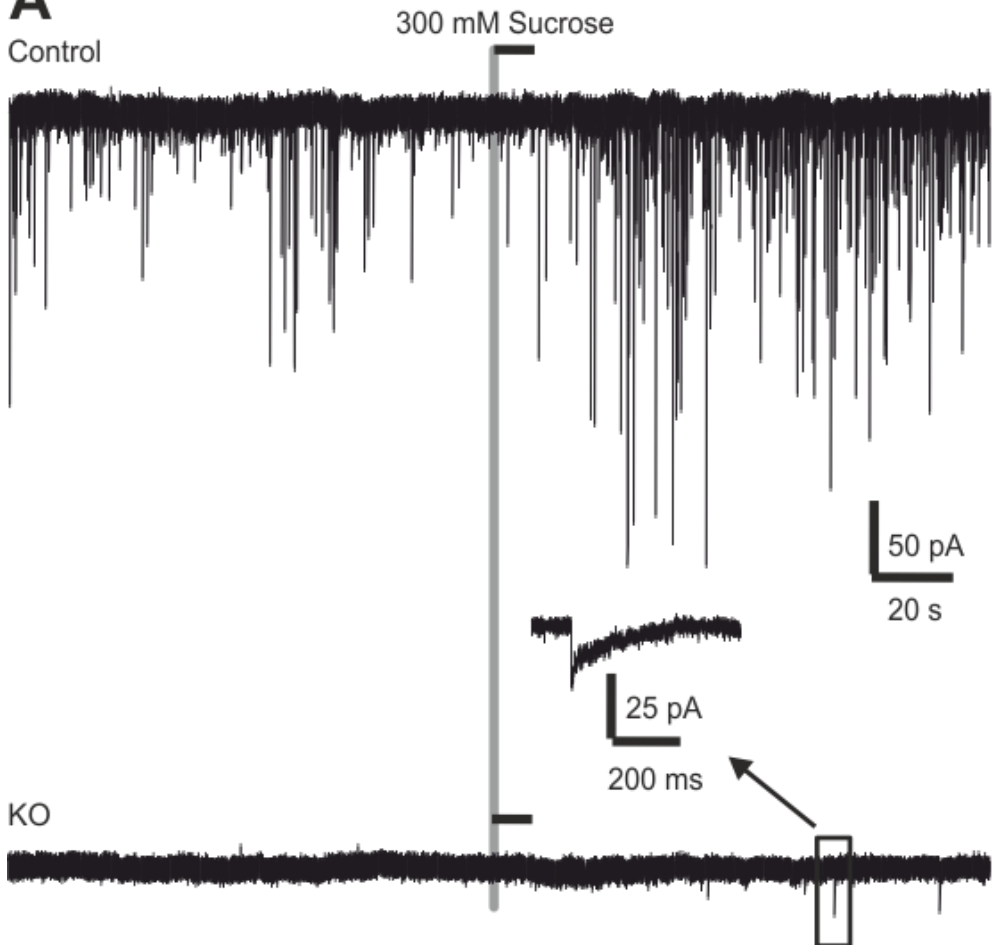

B
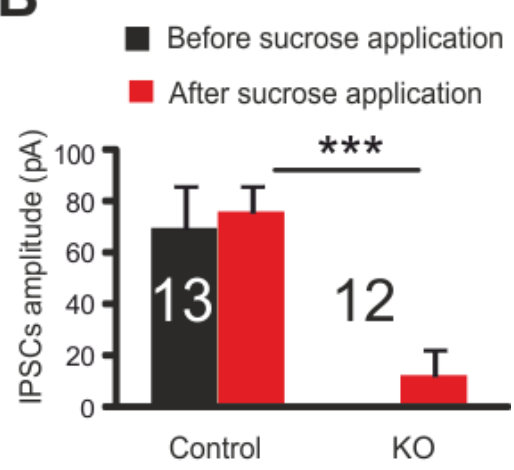

C

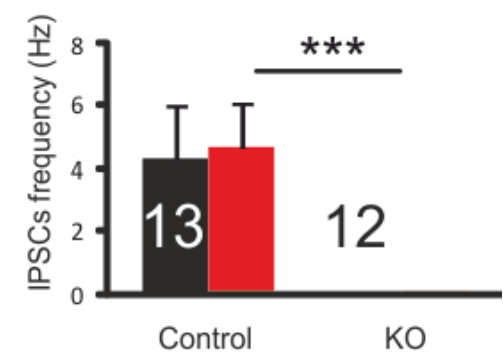

Figure 3.12 cVIAAT hypoglossal motoneurons lacked inhibitory transmission.

(A) A representative sIPSCs trace (upper) was recorded from a control hypoglossal motoneuron in the presence of CNQX, DL-AP5, Zolpidem. The grey line indicates the time point when sucrose was applied in the bath for $10 \mathrm{~s}$. The trace below is the representative sIPSCs trace recorded from cVIAAT KO hypoglossal motoneuron showed complete loss of sIPSCs except 1-2 detected only after sucrose application (box in lower trace). (B) The averaged sIPSCs amplitude before (black) and after (red) sucrose application, from control and CVIAAT KO hypoglossal motoneurons, are significantly different between genotypes but not with sucrose application. (C) Similarly, the averaged sIPSCs frequencies before (black) and after (red) sucrose application from control and CVIAAT KO hypoglossal motoneurons are significantly different between genotypes but not with sucrose application. Number inside and above the bars in $\mathrm{KO}$ and their control represents the neurons studied. 


\subsubsection{Glycinergic currents are reduced in cVIAAT KO hypoglossal motoneurons}

Glycine transmission is required for clustering of functional glycine receptors on postsynaptic side in-vitro (Kirsch \& Betz, 1998; Levi et al., 1998) and in vivo (Yamanaka et al., 2013). Since inhibitory transmission was almost absent in cVIAAT KO hypoglossal motoneurons, eIPSCs were recorded from cVIAAT KO hypoglossal motoneurons and their controls to check the presence of functional postsynaptic glycinergic and GABAergic receptors. The average amplitude of glycinergic eIPSC in cVIAAT hypoglossal motoneurons (3.61 $\pm 0.64 \mathrm{nA})$ was significantly reduced compared to their controls (5.28 $\pm 0.39 \mathrm{nA}$; Fig. 3.13B). However, the average amplitude of GABAergic eIPSC in cVIAAT hypoglossal motoneurons $(3.17 \pm 1.15 \mathrm{nA})$ did not change compared to their controls $(2.55 \pm 0.97 \mathrm{nA}$; Fig. 3.13D). 
A Glycine application

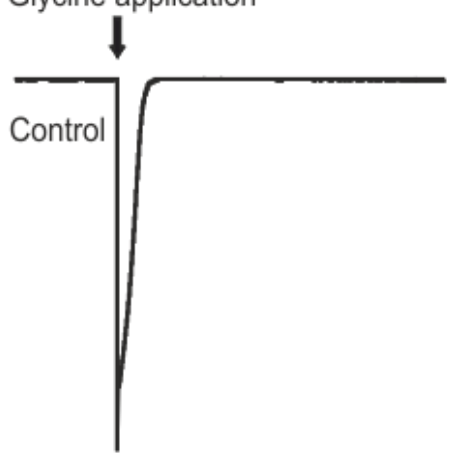

C

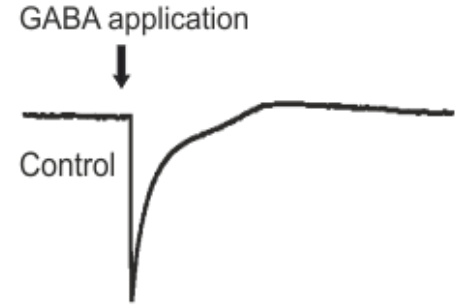

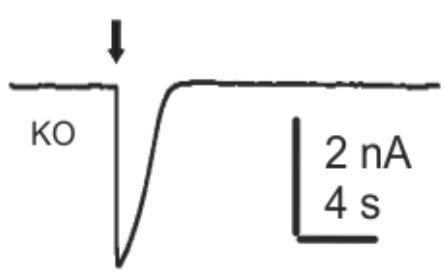

B
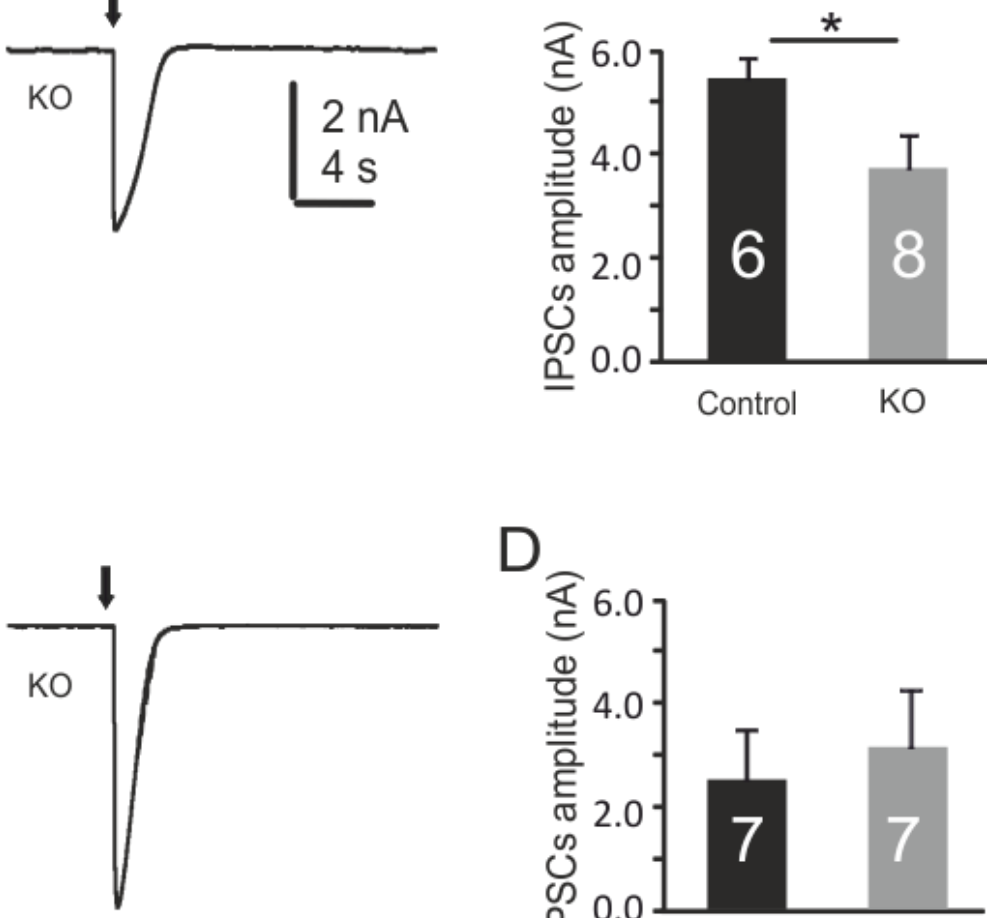

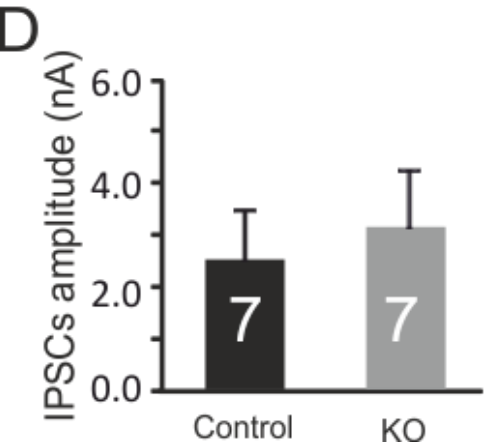

Figure 3.13 Glycinergic postsynaptic currents are reduced in hypoglossal motoneurons.

(A) An averaged representative glycinergic eIPSC of four consecutive glycine applications from a control (left) and cVIAAT KO hypoglossal motoneuron (right) are shown. The arrow indicates $1 \mathrm{mM}$ glycine application to both, the control and the CVIAAT KO recordings. (B) The averaged amplitude of glycinergic eIPSCs in CVIAAT KO hypoglossal motoneurons was significantly lower compared to their controls. (C) An averaged representative GABAergic elPSC of four consecutive GABA applications from a control (left) and cVIAAT KO hypoglossal motoneuron (right) are shown. Arrow indicates 10 mM GABA applications to both, the control and the cVIAAT KO recordings. (D) The averaged GABAergic elPSCs in CVIAAT hypoglossal motoneurons was similar compared to their controls. Number inside the bars represents the neurons studied. 


\subsubsection{Diaphragms of conditional VIAAT KO embryos are underdeveloped}

Inhibitory transmission was reduced in the CVIAAT KO hypoglossal motoneurons similar to spinal motoneurons of constitutional VIAAT KO embryos (Saito et al., 2010). In the VIAAT KO embryos, diaphragms have reduced phrenic nerve innervations compare to their controls, and they also do not breathe like the CVIAAT KO embryos (Fogarty et al., 2013b). Therefore, diaphragms of cVIAAT KO embryos and their controls were studied to check if there was any anatomical difference between genotypes. The cVIAAT KO diaphragm was smaller and wrinkled compared to their controls (Fig. 3.14A-B). The average diameter of the cVIAAT KO hemi-diaphragms $(1.76 \pm 0.17 \mathrm{~mm})$ was significantly smaller compared to its control hemidiaphragms (2.06 $\pm 0.16 \mathrm{~mm}$; Fig. 3.14C). The average surface area of the cVIAAT KO hemidiaphragms $\left(6.57 \pm 0.41 \mathrm{~mm}^{2}\right)$ was significantly smaller compared to its control hemidiaphragms (11.77 $\pm 0.52 \mathrm{~mm}^{2}$; Fig. 3.14D).
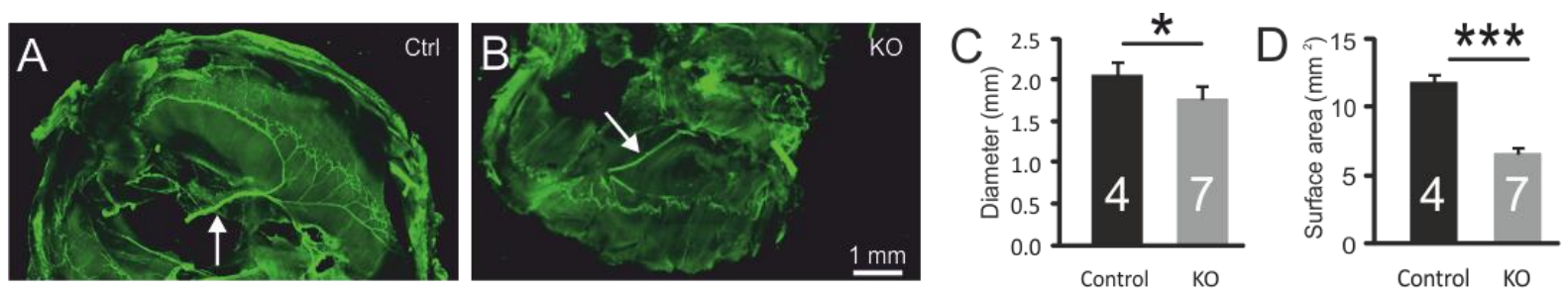

Figure 3.14 Whole-mounts neurofilament immunostaining on hemi-diaphragm.

(A) A representative control hemi-diaphragm innervated by phrenic main trunk (arrow) and its branches is shown. (B) A representative CVIAAT KO hemi-diaphragm innervated by distorted phrenic main trunk (arrow) and its branches is shown. (C) The averaged diameter of cVIAAT KO hemidiaphragm and their controls are shown. (D) The averaged surface area of CVIAAT KO hemidiaphragm and their controls are shown. Numbers inside bars are number of embryos studied. 


\subsection{Characterization of glycinergic neurons in the pre-Bötzinger complex}

The characterization of any neuronal populations including inhibitory neurons is a important step toward understanding the neuronal network and overall structure and functions of brain regions (Shino et al., 2011). Since glycinergic neurons in the preBötC are involved in respiratory pattern modulation (Winter et al., 2010); they were characterized by whole-cell Patch-clamp and dye filling experiments to understand their larger role in respiratory network.

\subsubsection{Rhythmic glycinergic neurons are primarily inspiratory}

To gather further information about glycinergic and potentially dual transmitting neurons in the preBötC, fluorescently labeled preBötC glycinergic neurons from the GlyT2-EGFP mice were characterized. $31 \%(\mathrm{~N}=28 / 91)$ glycinergic neurons were identified as respiratory glycinergic neurons that received the rhythmic inputs during respiratory bursts (Fig. 3.15A, upper trace). These glycinergic neurons also received rhythmic inputs in current-clamp (Fig. 3.15B). However, many glycinergic neurons $69 \%(\mathrm{~N}=63 / 91)$ did not receive any rhythmic input, neither in voltage-clamp (Fig. 3.15C) nor in current-clamp (Fig. 3.15D) and were classified as non-rhythmic glycinergic neurons (Fig. 3.15E). 

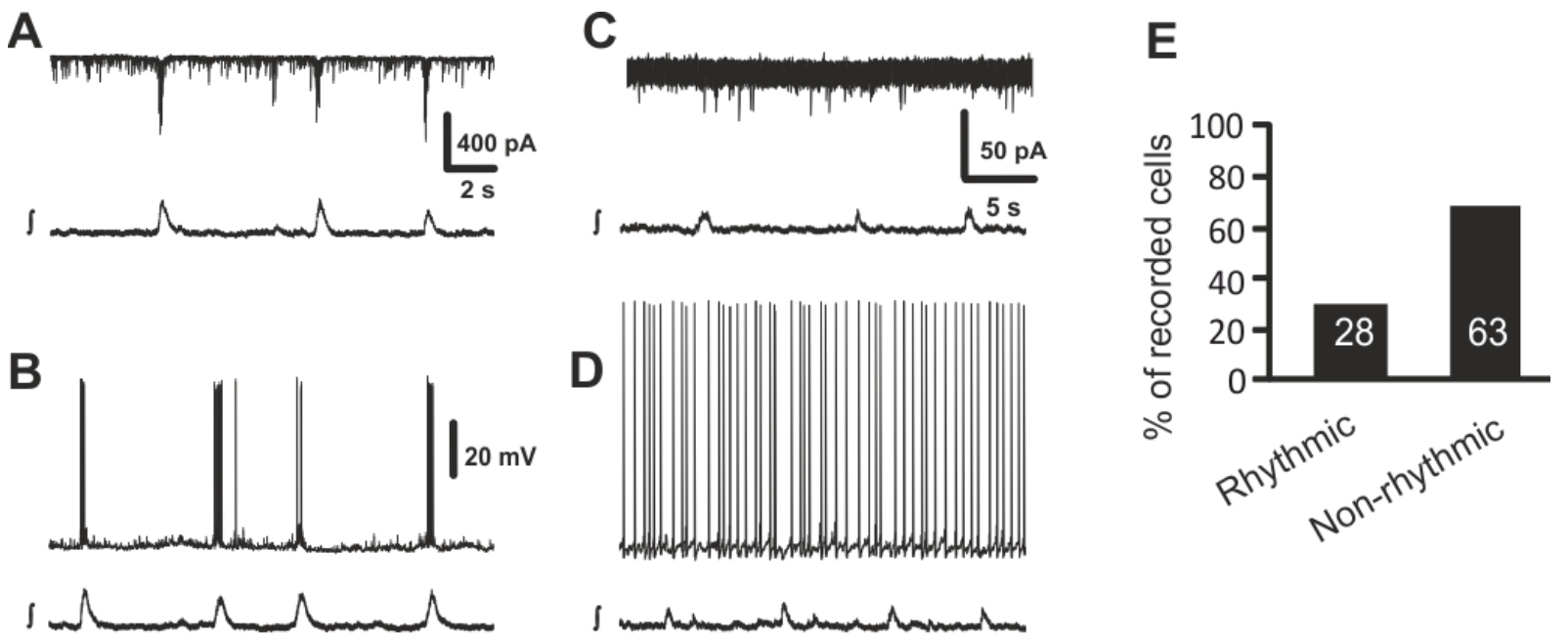

Figure 3.15 Glycinergic neurons receive the respiratory rhythmic inputs.

(A) A representative trace recorded from a glycinergic neuron that received synaptic inputs between respiratory rhythms and more synaptic inputs during respiratory rhythmic (upper trace) is shown. The below trace recorded in the preBötC showed an integrated mass activities of all neurons. (B) A representative membrane potential trace recorded from the same glycinergic neuron (as shown in Fig. A) received synaptic inputs between respiratory rhythms and additional excitatory inputs during respiratory rhythm led to burst, is shown. (C) A representative trace recorded from a non-rhythmic glycinergic neuron that receives spontaneous but not rhythmic synaptic inputs (upper trace) is shown. The trace below recorded from the preBötC showed integrated mass activities of all neurons. (D) A representative membrane potential trace recorded from the same glycinergic neuron (as shown in Fig. C), also shows that it does not receive any additional synaptic input during respiratory rhythm. (E) Summarized results from recorded glycinergic neurons are shown. Numbers inside bar represent the number of neurons. 


\subsubsection{Rhythmic glycinergic neurons receive excitatory and inhibitory inputs}

Rhythmic glycinergic neurons in the preBötC receive both excitatory and inhibitory inputs during inspiratory rhythms; however, these inputs have not been characterized in details (Winter et al., 2009). The low chloride pipette solution (intracellular solution 3) allows to differentiate between excitatory and inhibitory inputs (Winter et al., 2009). Using the same pipette solution $(\mathrm{Ecl}=-87 \mathrm{mV})$, glycinergic neurons were patched and recorded at $-70 \mathrm{mV}$ and $-50 \mathrm{mV}$. The inhibitory currents were outward at both potentials, however, they were not visible at $-70 \mathrm{mV}$ due to low driving force (Fig. 3.16A), but became clearly visible at -50 $\mathrm{mV}$ (Fig. 3.16A1) while excitatory currents remained inward at both potentials. In currentclamp, these glycinergic neurons showed weak depolarization leading to rhythmic APs (box in Fig. 3.16B). In contrast, some rhythmic glycinergic neurons when held at $-50 \mathrm{mV}$, showed only excitatory rhythmic inputs (Fig. 3.16C1; expansion of 3.16C). In current-clamp, these glycinergic neurons showed strong depolarization leading to strong rhythmic burst (box in Fig. 3.16D). Based on these results, 36\% $(N=5 / 14)$ rhythmic glycinergic neurons received both excitatory and inhibitory rhythmic inputs whereas $64 \%(N=9 / 14)$ received only excitatory rhythmic inputs (Fig. 3.16E). 

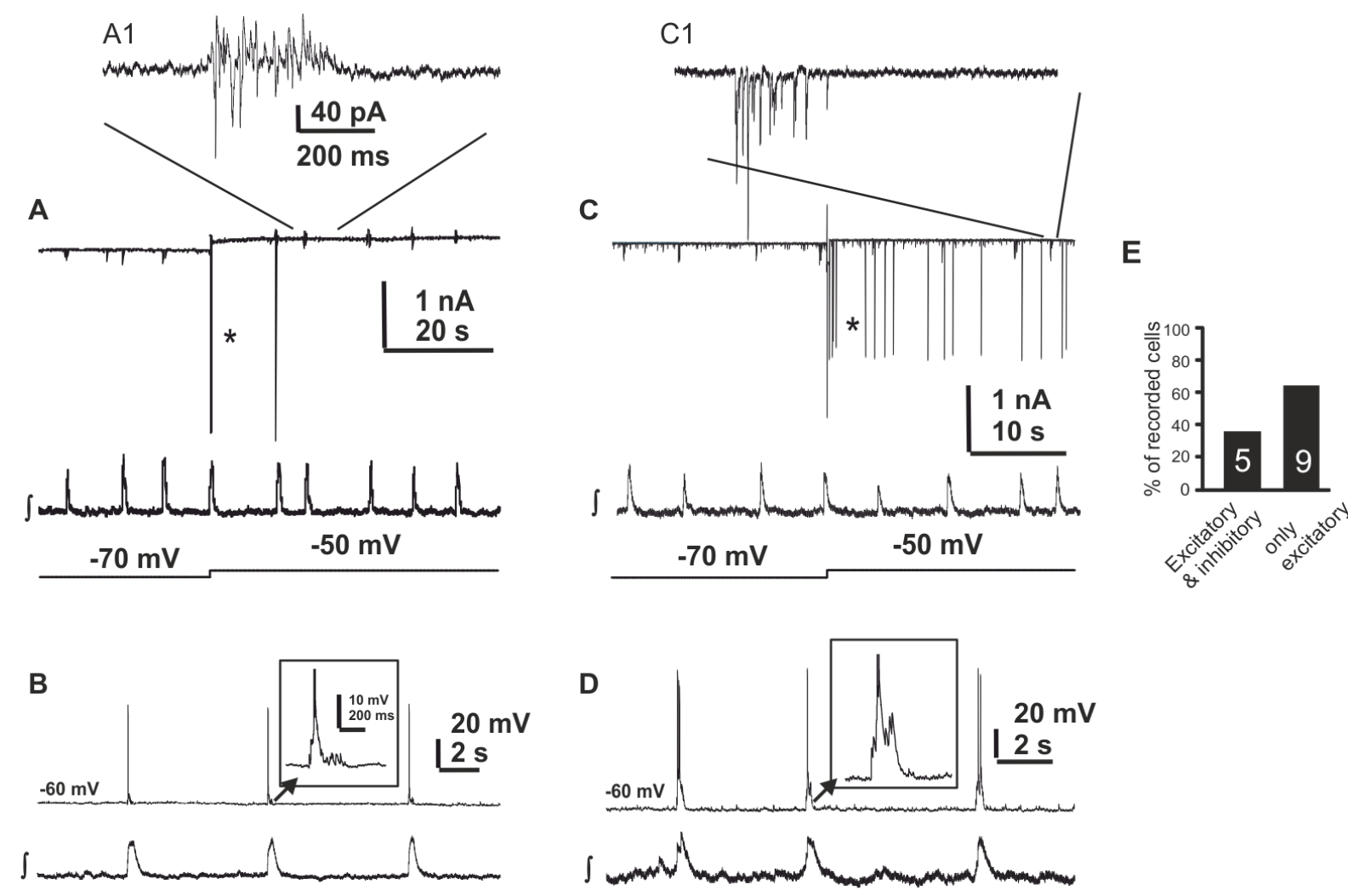

Figure 3.16 Glycinergic neurons received excitatory and inhibitory rhythmic inputs.

(A) A representative membrane current trace recorded from a glycinergic neuron at different holding potentials $(-70 \mathrm{mV}$ and $-50 \mathrm{mV})$ is shown. Low chloride intracellular solution $(\mathrm{Ecl}=-87 \mathrm{mV})$ was used to differentiate between excitatory and inhibitory inputs. This glycinergic neuron received both excitatory (inward) and inhibitory (outward) inputs during rhythmic bursts (see Figure A1). Middle trace recorded from the preBötC showed the mass activity from all neurons. The trace below shows neuron holding potential. (B) A representative membrane potential trace recorded from the same neuron is shown. Receiving of excitatory rhythmic inputs led to APs; the inset show the enlarged view of proceeding AP. Bottom trace recorded from the preBötC showed the mass activity from all neurons. (C) A representative membrane current trace recorded from another glycinergic neuron at same holding potentials $(-50 \mathrm{mV})$ is shown. Receives only excitatory (inward) inputs during respiratory rhythm (see inset $C 1$, scale bar same as $A 1$ ). (D) A representative membrane potential trace recorded from the same neuron (as Fig C) showed receiving of strong excitatory rhythmic inputs leading to burst; the inset shows the enlarged view of preceding AP (same scale bar same as inset B). (E) Summarized results from recorded glycinergic neurons area shown. Asterisks indicates APs driven current in figure $A$ and $C$. 


\subsubsection{Glycinergic neurons show pacemaker properties}

Glutamatergic neurons are considered as main pacemaker cells in the preBötC. However recent findings suggest, glycinergic neurons in the preBötC bear pacemaker properties (Morgado-Valle et al., 2010). Ectopic burst, voltage sags $\left(\mathrm{V}_{\text {sags }}\right)$, and rebound APs are also considered as properties of a pacemakers neuron. Therefore, it was tested whether glycinergic neurons in the preBötC also generate ectopic bursts. Indeed, glycinergic neurons were capable of generating ectopic bursts in addition to rhythmic bursts $(62.5 \%$; $N=15 / 24$; Fig. 3.17A). While many glycinergic neurons produced only rhythmic burst (29.17\%; $N=7 / 24)$, some glycinergic neurons produced ectopic burst without any rhythmic preBötC burst (8.33\%; $N=2 / 24 ;$ Fig. 3.17B). 

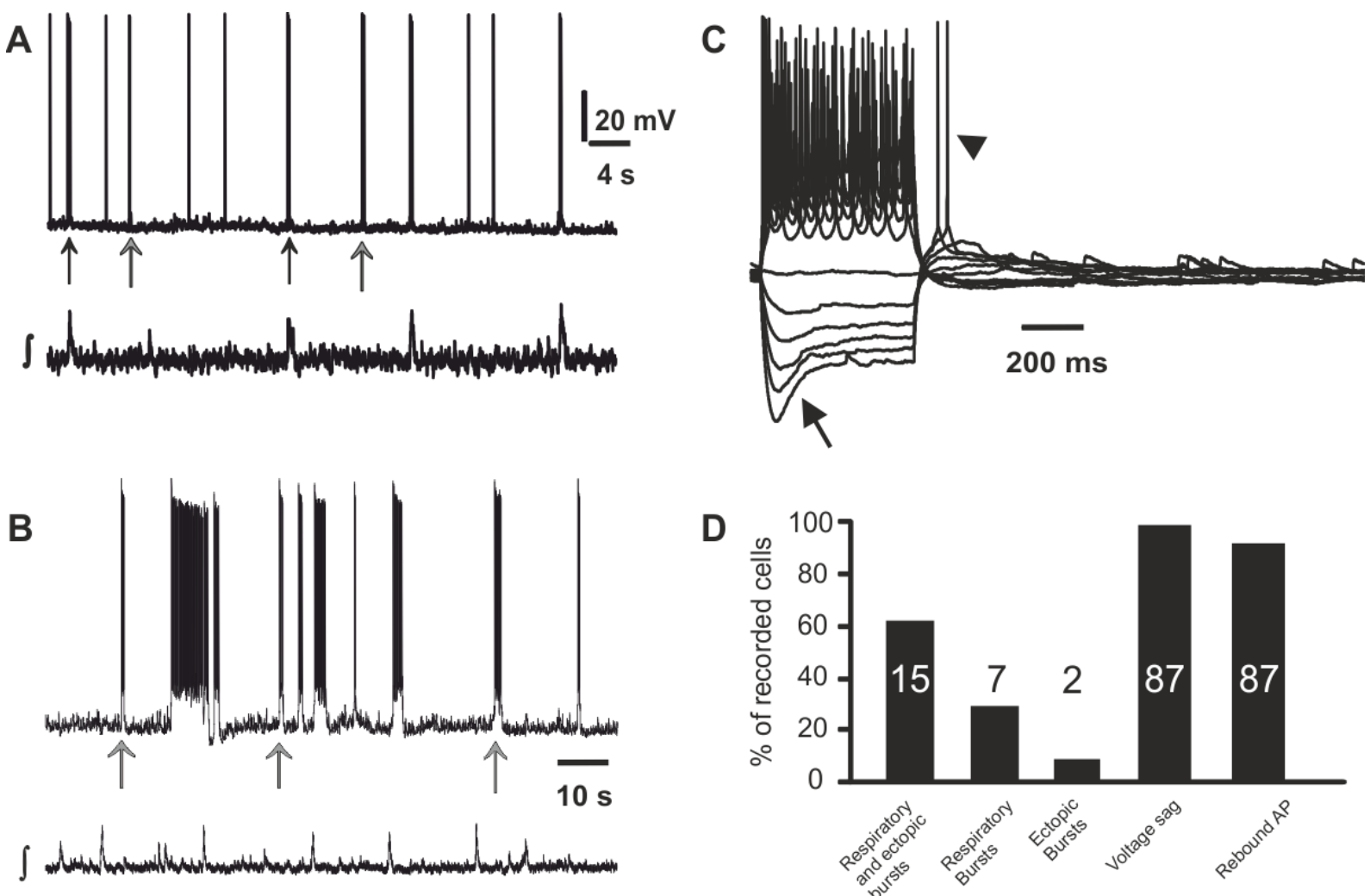

Figure 3.17 Glycinergic neurons show pacemaker properties.

(A) A representative membrane potential trace recorded from a glycinergic neuron shows ectopic burst (grey arrows) and rhythmic bursts (black arrows). The bottom trace recorded from the preBötC shows the integrated mass activity of all neurons. (B) A representative membrane potential trace recorded from another glycinergic neuron shows ectopic burst (grey arrows) but does not show rhythmic burst. (C) A representative membrane potential trace recorded from a glycinergic neuron in response to step-pulse DC (from $-320 \mathrm{pA}$ to $180 \mathrm{pA}$ with $50 \mathrm{pA}$ step, $500 \mathrm{~ms}$ ) shows $\mathrm{V}_{\text {sag }}$ (see arrow) and rebound APs (see arrow head). (D) The summarized results from recorded glycinergic neurons are shown. The numbers inside the bar represent recorded cells. Scale bars on y-axis are same in Fig. A-C.

Type 2 inspiratory neurons in the preBötC have been shown to have $V_{\text {sags }}$ (due to $I_{H}$ current) upon hyperpolarization and rebound APs (due to reactivation of sodium current) upon returning to the resting membrane potential (Rekling et al., 1996). It was checked whether a glycinergic neuron in the preBötC also had these properties. In fact, the glycinergic neuron showed $\mathrm{V}_{\text {sags }}$ with hyperpolarizing current (arrow in Fig. 3.17C) and rebound AP (arrow head in Fig. 3.17C) after returning to the base line, with an applied step-pulse DC (from -320 pA to 180 pA with 50 pA step for 500 ms). The majority of glycinergic neurons exhibited $\mathrm{V}_{\text {sags }}$ (97.70\%; $\mathrm{N}=85 / 87$ ) and rebound APs (94.25\%; $\mathrm{N}=82 / 87 ;$ Fig. 3.17D). 


\subsubsection{Synaptic coupling between glycinergic neurons}

To study the synaptic strength between glycinergic neurons in the preBötC, paired recordings were performed. Glycinergic neurons (both cell 1 and cell 2 in Fig. 3.18 A-B) received spontaneous synaptic inputs, however, that did not correspond to the activity (AP) in the other glycinergic neuron of the pair (Fig. 3.18 A-B). Additionally, the synaptic couplings between glycinergic neurons were checked with depolarizing DC stimulation (Fig. 3.18 C-D), which also could not show synaptic coupling. The paired-recordings (16 from 6 mice) between nearby glycinergic neurons in the preBötC did not reveal any uni- or bi-directional synaptic coupling.

A
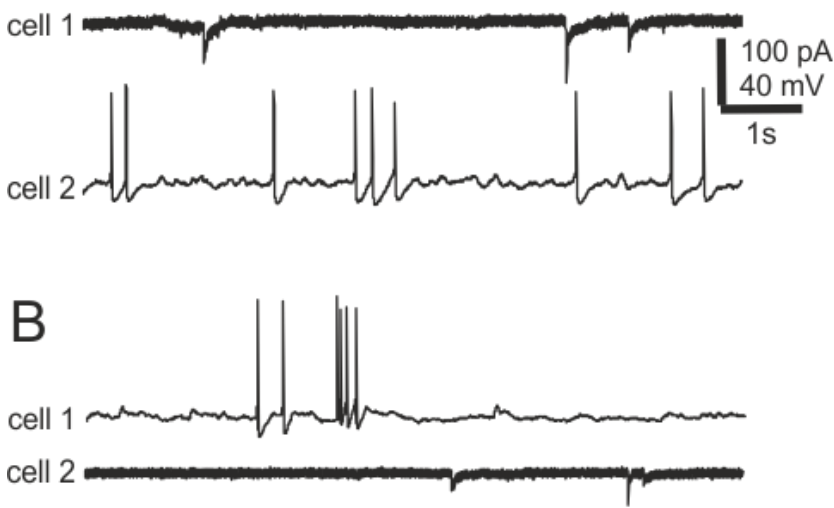

C
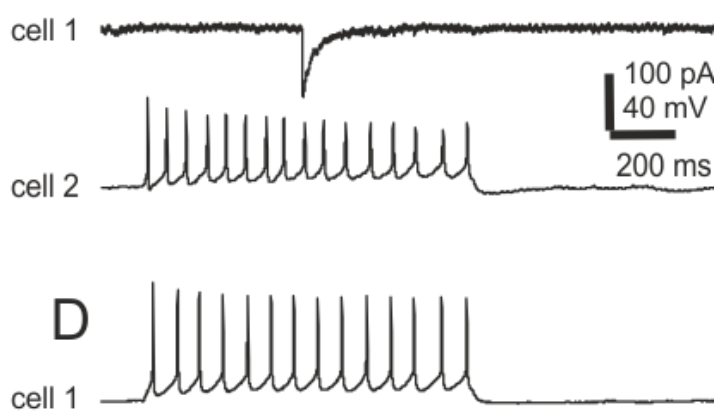

cell 2

Figure 3.18 Paired recording from two glycinergic neurons in the preBötC.

(A) A representative paired recording trace recorded from two glycinergic neurons, where cell 1 (in voltage-clamp) is receiving sIPSCs and cell 2 (in current-clamp) shows spontaneous APs. However, there was no correlation between their activities. (B) The same neuronal pair when held in opposite clamping, means, then cell 1 was in current-clamp and cell 2 in voltage-clamp, still there was no correlation between them. (C) Another representative paired recording from another glycinergic neurons is shown, where cell 2 (in current-clamp) was stimulated with step-pulse DC (110 pA for 500 $\mathrm{ms})$ to observe correlated sIPSCs in cell 1 which was held in voltage-clamp. APs in cell 2 do not induce corresponding sIPSCs in cell 1. (D) The same neurons when place in opposite-clamping and cell 1 was stimulated with the same step-pulse DC, that also does not elicit any corresponding sIPSC in cell 2. 


\subsubsection{Morphology of glycinergic neurons}

Glycinergic neurons in the preBötC have been studied via electrophysiology (Morgado-Valle et al., 2010; Rahman et al., 2013) and calcium sensitive dye (Winter et al., 2009). However, their detailed morphological characterization via dye filling has not been done. Here, glycinergic neurons were identified by EGFP expression in GlyT2-EGFP mice and filled with different dye (Alexa 568 and Alexa 647) to study their processes (axons and dendrites). In a preliminary set of experiments, glycinergic neurons $(\mathrm{N}=55)$ in the preBötC were filled with Alexa 568. Dendritic projections of these glycinergic neurons were distributed proximally to the soma while the axon was difficult to be distinguished (Fig. 3.19 A-B). Their soma were either pyramidal or spindle shape. Additionally, pairs of glycinergic neurons $(N=14)$ were filled with two different dyes (Alexa 568 and Alexa 647) to identify projections of their processes. Dendritic projections of these glycinergic neurons spread proximal to the soma (Fig. 3.19 C-D) whereas a process (presumably axon) was projected far away ( $>400 \mu \mathrm{m}$ ) from soma to ipsilateral dorsal side (arrow in Fig. 3.19D). 

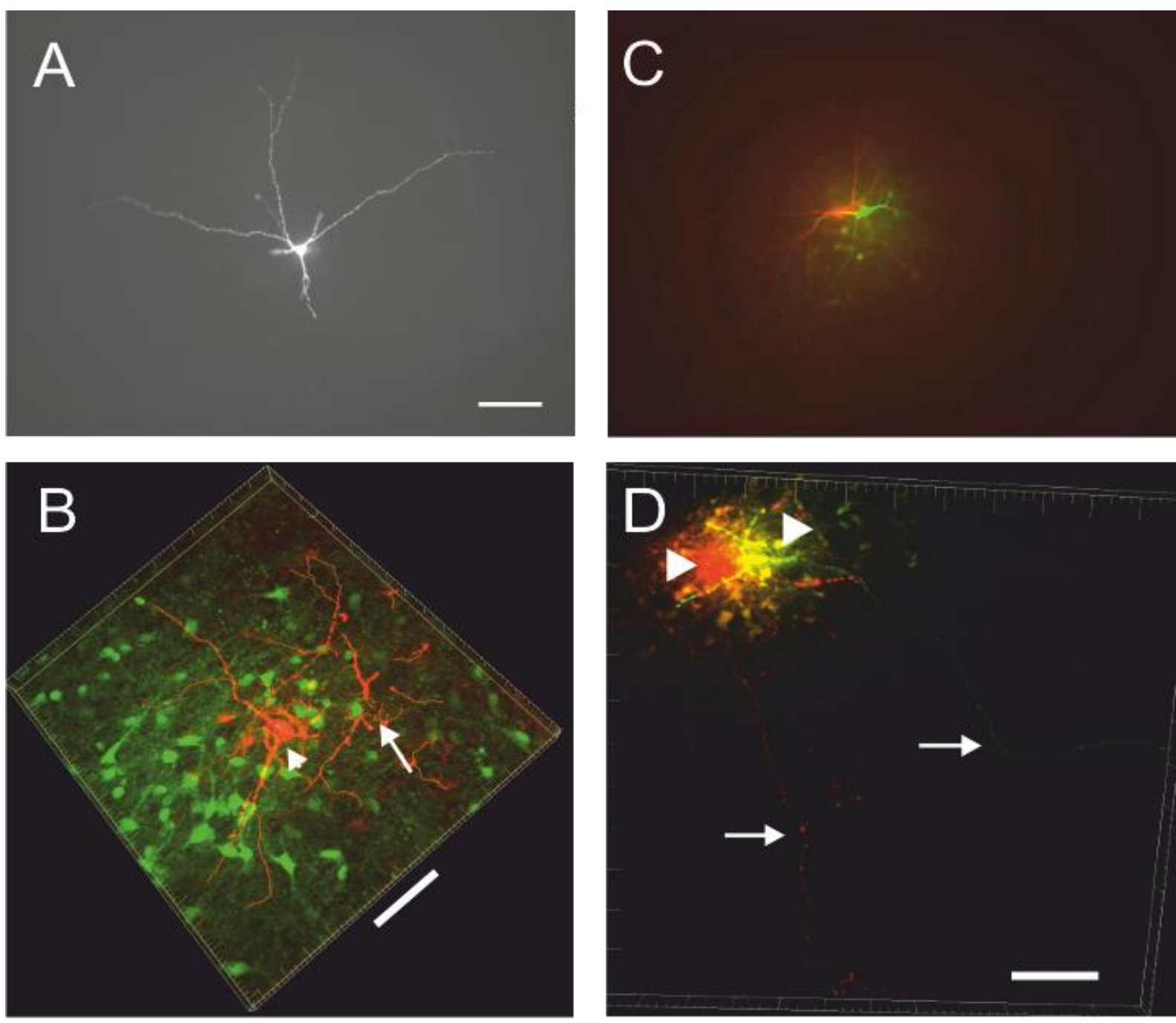

Figure 3.19 Processes of glycinergic neurons in the preBötC.

(A) A representative pyramidal glycinergic neuron filled with alexa 568 is shown. (B) A representative maximum intensity projection image shows two filled glycinergic neurons (red; arrow head and arrow point to soma). The soma of one filled glycinergic neuron (see arrow) came out along with the patch pipette. All green neurons are glycinergic neurons. (C) Representative spindle shape glycinergic neurons; one filled with Alexa 568 (green) and other Alexa 647 (red) are shown. (D) A representative maximum intensity projection image shows soma of two filled glycinergic neurons (arrow heads) and their axonal projections (arrows). Scale bars: $100 \mu \mathrm{m}$ (A and C), $66 \mu \mathrm{m}$ (B), $80 \mu \mathrm{m}$ (D). 


\section{Discussion}

\subsection{Role of co-transmission in the respiratory network}

In this thesis, co-transmission of GABA and glycine in the preBötC has been studied using electrophysiology, sc-RT-PCR and a cVIAAT KO mouse model. The results of this study show for the first time that GABA and glycine are co-transmitted in the pre-Bötzinger complex. Decay time constants and amplitudes of mIPSCs were analyzed to estimate the percentage of mixed-mIPSCs resulting from co-release of GABA and glycine compared to overall inhibitory transmissions (Rahman et al., 2013). Using the optimized method the " $\Sigma E P F "$, mixed-mIPSCs were observed up to $46.8 \%$ and $36.1 \%$ in glycinergic neurons and rhythmic glycinergic neurons respectively. These results fall within the range of previously reported studies in other brain regions (Jonas et al., 1998; Nabekura et al., 2004; Awatramani et al., 2005). The sc-RT-PCR data has also shown higher number (64\%) of glycinergic neurons in the preBötC that co-express both glycinergic and GABAergic neuronal markers, which also support the co-transmission in the preBötC. Similarly, GABAergic neurons in the preBötC also co-express markers for both GABAergic and glycinergic neurons in GAD67-GFP neonate mice (Koizumi et al., 2013). The drastically reduced VIAAT expression in the preBötC and hypoglossal nucleus of the cVIAAT KO embryos strongly suggest that GlyT2-cre was expressed thereby eliminated VIAAT from most of inhibitory neurons (>90\%) during embryonic stage. Further, absence of sIPSCs in cVIAAT KO hypoglossal motoneurons additionally confirms wide expression of GlyT2-cre through embryonic brainstem development. These findings together underpin that the co-transmission is present in the preBötC and support previous findings of co-transmission in other brainstem regions.

The inhibitory transmission in the VRC including the preBötC is involved in shaping and patterning of the respiratory rhythm (Hayashi \& Lipski, 1992; Busselberg et al., 2001). Further, GABA and glycine co-transmission mediated inhibition in the NTS is postulated to play a role in the fast neuronal inhibition during respiratory reflexes (Dufour et al., 2010). Additionally, GABA and glycine mediated inhibitory transmission is hypothesized in synchronization of hypoglossal nerve output (Sebe et al., 2006). Based on above findings, it can be hypothesized that the inhibitory co-transmission in the preBötC could be involved in 
synchronization of inspiratory and expiratory activity. The co-release mediated inhibition is faster compared to single transmitter mediated inhibition which can be accomplished due to two reasons. (1) Mixed transmission compared to pure GABA transmission can steeply increase the membrane conductance with faster activation of GlyR (Russier et al., 2002); (2) GABA co-release with glycine may act as co-agonist on GlyR and thereby shorten inhibition time by decreasing the decay time constant as shown in the auditory network (Lu et al., 2008). GABA and glycine co-release and thereby co-transmission in the preBötC may be an optimal mode of inhibition to achieve fast synchronized inhibition as reported in other regions (Russier et al., 2002; Dufour et al., 2010).

Lack of breathing and loss of phrenic nerve activity in VIAAT KO embryos are already reported, which lack inhibitory transmission in spinal motoneurons (Fujii et al., 2007; Saito et al., 2010). Even a selective elimination of GABAergic transmission causes the loss of phrenic nerve output (Fujii et al., 2007). In consistence with these studies, the CVIAAT KO embryos did not breathe and their hypoglossal motoneurons did not receive any inhibitory input as well. In addition, VIAAT expression was also dramatically reduced in hypoglossal nucleus and in the preBötC and most likely other brainstem regions of the CVIAAT KO as GlyT2 is reported to be expressed in hindbrain and spinal cord (Zafra et al., 1995). Taken together, these results strongly suggest that VIAAT was removed from both glycinergic and GABAergic terminals in the area of hypoglossal nucleus and the preBötC. Since the VIAAT removal was dependent on GlyT2-cre expression, it looks like that GlyT2 was expressed is all inhibitory neurons. Therefore it can be concluded that GlyT2 is an early marker of all inhibitory neurons in the brainstem during embryonic stage.

Despite absence of sIPSCs in hypoglossal motoneurons of CVIAAT KO embryos and dramatic reduction of VIAAT staining in this region as well as in the preBötC, spontaneous bursting activities in the preBötC were present in rhythmic slices. Additionally, the cVIAAT KO embryos did not breathe. These results fall in line with previous publication which showed that rhythmic phrenic nerve output is absent in the brainstem-spinal cord preparation made from VIAAT KO embryos even though neurons in the pFRG (another respiratory rhythm generator) are capable of firing AP (Fujii et al., 2007). Therefore, these findings together ratify the observations that inhibitory transmission is not obligatory for the generation of rhythmic activity in the preBötC (Shao \& Feldman, 1997) and the network of excitatory 
pacemaker neurons is sufficient for respiratory rhythm generation (Wallen-Mackenzie et al., 2006b). Rhythmic bursting activity in the preBötC is increased with bicuculline and strychnine application as reported in the neonate RVLM (Onimaru et al., 1990), which indicates that GABA and glycine indeed are inhibitory during this age (E18.5) as suggested before (Feng et al., 1998; Greer \& Funk, 2005). Interestingly, it is known that inhibitory transmission is required for coupling of two oscillators - the pFRG and the preBötC to produce normal respiratory rhythm (Thoby-Brisson et al., 2009; Lal et al., 2011). Since inhibitory transmission was absent in CVIAAT KO as well as VIAAT KO embryos, the most likely reason for respiratory failure and subsequent death of these embryos was lack of coupling between the PFRG and the preBötC. However, it remains to be studied whether cotransmission of GABA and glycine or GABAergic or glycinergic transmission alone is sufficient for this coupling.

\subsection{Loss of inhibition affects function of hypoglossal motoneurons}

Hypoglossal motoneurons are involved in regulation of breathing by control of tongue muscles. Synchronized hypoglossal output, which is in-phase with phrenic nerve activity, depends on intact inhibitory transmission in the neonate rodents (Sebe et al., 2006). The cVIAAT KO hypoglossal motoneurons did not receive any SIPSC, which is similar to the previous study that shows complete loss of sIPSCs in VIAAT KO spinal motoneurons (Saito et al., 2010). Similarly, sIPSCs are completely absent in $>80 \%$ VIAAT KO cultured spinal motoneurons (Wojcik et al., 2006). Furthermore, any non-physiological reduction in GABA and/or glycine transmission reduces the number of hypoglossal as well as phrenic motoneurons as shown in VIAAT KO, GAD67 KO and gephyrin KO embryos (Banks et al., 2005; Fogarty et al., 2013a; Fogarty et al., 2013b). These findings together reflect that GABA and glycine transmission are important for the development of motoneurons including hypoglossal motoneurons.

Amplitudes of glycinergic eIPSCs in CVIAAT KO hypoglossal motoneurons were reduced while GABAergic remained unchanged. This fits with a previous study that shows that amplitude as well frequencies of glycinergic mIPSCs were drastically reduced in VIAAT KO spinal motoneurons (Wojcik et al., 2006). The reduced glycinergic elPSCs must be due to the 
reduction of functional glycine receptors on cVIAAT KO as well as VIAAT KO hypoglossal motoneurons as glycine transmission is required for glycine receptors clustering which is shown by in vitro (Kirsch \& Betz, 1998; Levi et al., 1998) and in vivo experiments (Yamanaka et al., 2013). The bath application of sucrose, which increases the release probability of neurotransmitters (Rosenmund \& Stevens, 1996), has produced barely 1-2 sIPSCs in cVIAAT KO hypoglossal motoneurons. These 1-2 sIPSCs looked similar to a GABAergic IPSCs based on a decay kinetic $\left(\tau_{\text {decay }} \sim 65 \mathrm{~ms}\right.$ ) as shown in the preBötC of GlyT2-EGFP mice (Rahman et al., 2013). Further, their GABAergic nature could also be speculated because VIAAT was supposedly removed from all glycinergic neurons but not from all inhibitory neurons as $~ 5 \%$ VIAAT expression was still present in the hypoglossal nucleus. Additionally, these rare sIPSCs would be GABAergic as the leftover sIPSCS in VIAAT KO spinal motoneurons were also GABAergic (Wojcik et al., 2006).

The membrane capacitance $\left(C_{m}\right)$ of cVIAAT KO hypoglossal motoneurons was significantly lower compared to their control. In general, the $C_{m}$ depends on the neuronal surface area including dendritic and axonic branches. The somatic areas of hypoglossal motoneurons do not change due to disturbance in inhibitory transmission, while any change in their axonic and dendritic branches is unknown (Banks et al., 2005; Fogarty et al., 2013a; Fogarty et al., 2013b). However, it is shown in spinal motoneurons that axotomy reduces the $C_{m}$, therefore, the lower $C_{m}$ of $c$ VIAAT KO hypoglossal motoneurons could be due to their reduced axonic and dendritic branches (Yamuy et al., 1992). The reduced axon may be the most likely reason for low $C_{m}$ because the decrease in phrenic nerve branching on diaphragm has been already observed in VIAAT, GAD67, and gephyrin KO embryos (Banks et al., 2005; Fogarty et al., 2013a). The input resistance $\left(R_{\text {in }}\right)$ of cVIAAT KO hypoglossal motoneurons and their controls were comparable and are similar to the $R_{\text {in }}$ of embryonic phrenic motoneurons (Martin-Caraballo \& Greer, 1999). 


\subsection{Developmental variability in co-transmission of GABA and glycine}

In the cVIAAT KO embryos, hypoglossal motoneurons did not receive any GABAergic or glycinergic sIPSC which means GlyT2-cre was expressed and thereby removed VIAAT from all glycinergic and GABAergic neurons projecting to hypoglossal nucleus. The wide expression of GlyT2 in GABAergic neurons in addition to glycinergic neurons indicates that higher numbers (>90\%) of inhibitory neurons could be capable of filling and thereby co-releasing both GABA and glycine during embryonic stage. During first and second postnatal weeks of development, around 64\% glycinergic (Rahman et al., 2013) and 45\% GABAergic (Koizumi et al., 2013) neurons co-express both GABAergic and glycinergic neuronal markers. Further, mixed-mIPSCs were up to $46.8 \%$ in the preBötC glycinergic neurons, which is similar to rat spinal cord (Jonas et al., 1998) but lower than rat LSO (Nabekura et al., 2004). The higher mixed-mIPSCs in LSO may be due to the difference in the method as amplitudes instead of decay time constants of GABA and glycine component of mIPSCs are considered for mixedmIPSCs estimation. However, studies in adult mice do not show co-expression of GABAergic and glycinergic neuronal markers except in a subpopulation of GABAergic precursor cells in the cerebellum (Zeilhofer et al., 2005; Simat et al., 2007a; Simat et al., 2007b). Interestingly, mixed-IPSCs are also reduced in medial nucleus of the trapezoid body (MNTB) of adult rat (Awatramani et al., 2005). Taken together, these results strongly suggest that co-release and thereby co-transmission contribution is higher in embryonic stage compared to neonate and adult stage.

\section{Discrepancy between electrophysiology and single cell reverse transcription polymerase chain reaction}

Sc-RT-PCR analysis showed higher number of glycinergic neurons (64\%), which are capable of co-releasing both GABA and glycine, compared to mixed-mIPSCs (46.8\%) detected in glycinergic neurons by electrophysiology. This discrepancy could be due to either different expression or activation of postsynaptic receptors or synthesis and co-release of neurotransmitters in the presynaptic terminals. At postsynaptic side, the co-transmission depends on the subunits expression of $G_{A B A} R$ and GlyR with respect to location, composition, and stoichiometry. Further, the distribution of inhibitory receptors varies from synapse to synapse (Dugue et al., 2005), therefore, it is unlikely that all mixed synapses in this study had homogenous expression of both $G_{A B A} R$ and GlyR. A non-homogenous 
expression of $G_{A B A} R$ and GlyR would also reduce the detection of many co-release events, even with equal content of GABA and glycine. Interestingly, an equal amount of GABA and glycine at mixed synapses may activate more GlyR and less $G A B A_{A} R$ because a GlyR needs only one glycine molecule whereas a $G_{A B A} R$ needs two GABA molecules to be activated (Twyman \& Macdonald, 1991; Burkat et al., 2001). These conditions might have interfered with proper detection of mixed-mIPSCs; therefore, the actual \% of mixed-mIPSCs in the preBötC might be even more than being calculated.

On the presynaptic side, the content of GABA and glycine would be different in SVs due to heterogeneous expression of their synthesizing enzymes and transporters (GAD67, GAD65, GAT1, and GlyT2) in glycinergic neurons as the transmitter content in a SV strongly depends on their cytosolic concentration (Aubrey et al., 2007; Apostolides \& Trussell, 2013; Ishibashi et al., 2013). If the contribution of one of the component either GABA or glycine was below $30 \%$ in a mIPSC, this mIPSC would not have been detected as the mixed-mIPSCs (Rahman et al., 2013). The co-expression of GABA and glycine neuronal markers in glycinergic neurons do not eliminate the possibility of pure GABA or pure glycine SVs in their terminals as reported in some NTS neurons (Dufour et al., 2010).

Sc-RT-PCR revealed that $18 \%$ of glycinergic neurons express at least one GABAergic marker (GAD67, GAD65, or GAT1) but not GlyT2. This condition might have arisen due to two reasons. The first reason might be low level of GlyT2 mRNA which was not possible to be detected by the reverse transcription polymerase chain reaction as shown in rostral ventrolateral medulla of neonatal rat (Comer et al., 1999) and in another brainstem region known as prepositus hypoglossi nucleus which is responsible for horizontal gaze holding (Shino et al., 2008). 


\section{Conditional VIAAT KO is restricted to caudal brain regions}

Since GlyT2 promoter is restricted to brainstem and spinal cord during embryonic development (Zafra et al., 1995; Jursky \& Nelson, 1996) and its activity already starts around E11 (Adams et al., 1995), cre-mediated recombination at E18.5 was expected to occur in hindbrain only. Indeed, the reduced VIAAT expression was seen in hypoglossal nucleus and the preBötC but not in hypothalamus of cVIAAT KO embryos.

\subsection{Loss of GABAergic transmission causes omphalocele}

An omphalocele has been reported due to a loss of inhibitory transmission in VIAAT KO mouse models (Wojcik et al., 2006; Fujii et al., 2007; Oh et al., 2010; Saito et al., 2010). Likewise, this phenotype is also seen in KCC2 KO mouse model where inhibitory transmission is completely absent (Hubner et al., 2001). Therefore, the appearance of an omphalocele in all cVIAAT KO embryos and absence of sIPSC in cVIAAT KO hypoglossal motoneuron confirm the loss of both glycinergic and GABAergic transmissions cVIAAT KO embryos. This must have happened due to GlyT2-cre expression in all glycinergic and most of GABAergic neurons as a disturbance in GABAergic transmission alone causes the omphalocele only in $43 \%$ embryos (Saito et al., 2010) while a disturbance in glycinergic transmission does not cause the omphalocele at all (Feng et al., 1998; Gomeza et al., 2003b). The ubiquitous GlyT2-cre expression was more evident from highly reduced VIAAT expression and complete loss of sIPSCs in hypoglossal nucleus and neurons respectively. Additionally, cVIAAT KO embryos were kyphotic (Fig. 3.4A) that might have exerted additional pressure on intraabdominal muscles resulting into failure of abdominal wall closure as hypothesized in VIAAT KO embryos model (Saito et al., 2010).

Cleft palate is another non-neuronal defect, found in CVIAAT KO and other VIAAT KO (Wojcik et al., 2006; Fujii et al., 2007; Oh et al., 2010; Saito et al., 2010) and GAD67 KO mouse models (Asada et al., 1997; Condie et al., 1997; Kuwana et al., 2003; Tsunekawa et al., 2005; Fujii et al., 2007; Oh et al., 2010). In contrast, GAD65 KO mice (Asada et al., 1996) do not show cleft palate because sufficient GABA is synthesized from GAD67. These embryonic mouse models suggest that GABAergic transmission is obligatory for closure of the palate. The presence of cleft palate in cVIAAT KO embryos further emphasize that GlyT2-cre 
removed VIAAT from most inhibitory projections to hypoglossal nucleus, the preBötC and most likely from other hindbrain regions as well. Glycine is not required for palate closure because neither gephyrin KO nor GlyT2 KO mouse model exhibits cleft-palate (Feng et al., 1998; Gomeza et al., 2003b). Finally, these mouse models strongly demonstrate that GABA, but not glycine, is required for closure of palate. In fact GABA application in organotypic palate cultures, which are made from embryonic mice, rescue palate closure (Oh et al., 2010).

\subsection{Glycinergic neurons are integrated in the pre-Bötzinger complex}

Characterization of glycinergic neurons in the preBötC supports previous studies which have shown that glycinergic neurons receive respiratory inputs (Winter et al., 2009; MorgadoValle et al., 2010). Glycinergic neurons in the preBötC received both excitatory and inhibitory inputs during inspiratory rhythmic bursts which further support the previous finding of our lab (Winter et al., 2009). These finding together substantiate the idea of mutual inhibition between the preBötC and the BötC which is proposed as the core-mechanism for respiratory rhythm generation in adult rodents (Abdala et al., 2009; Lindsey et al., 2012; Smith et al., 2013). Previous studies found that glycinergic neurons in the preBötC show pacemaker properties (Morgado-Valle et al., 2010) or express molecular markers for pacemaker properties (Del Negro et al., 2002a; Del Negro et al., 2002b; Biel et al., 2009; Ramirez et al., 2011), but they were not quantified. Some of these properties, like $V_{\text {sag }}$ and rebound APs were quantified in glycinergic neurons of the preBötC which turned out to be a very common among of these neurons.

Synaptic coupling between glycinergic neurons was not detected by paired electrophysiological recordings in the preBötC of neonatal mice. This is in line with previously study which reported less than $10 \%$ of preBötC neurons of the neonatal mice show synaptic coupling (Rekling et al., 2000). These results together suggest, overall low synaptic coupling among preBötC neurons which were recorded in brain slices made from neonatal mice. The low synaptic coupling is not very surprising, as the paired recordings were made during first postnatal week (p1-p4) when chemical synapses between neurons are still in the process of development while electric coupling is prevalent (Bou-Flores \& 
Berger, 2001; Kiehn \& Tresch, 2002; Connors \& Long, 2004). The glycinergic neurons recorded for synaptic coupling, were present in the same coronal plane and very close to each other; however, the dye filling experiments of glycinergic neurons showed the axonic projections far away from their soma while dendritic projections in the proximity of their soma. Since axon leaves the proximity of soma and project ipsilateral towards the dorsal side as shown in neonatal rat (Koizumi et al., 2013), this may be another reason for low synaptic coupling between glycinergic neurons in the preBötC. The low synaptic coupling also could be due to axonic and dentritic projections of inhibitory neurons in rostro-caudal direction which is necessary for core microcircuitry between BötC and preBötC (Abdala et al., 2009). 


\section{Summary and Conclusion}

Inhibitory neurons in the pre-Bötzinger complex - a kernel for respiratory rhythm generation, are involved in the respiratory rhythm modulation. The co-transmission of GABA and glycine has been reported in different brain regions but not in the preBötC. The current study showed that GABA and glycine co-transmission is present in the neonatal mouse preBötzinger complex. The co-transmission mediated inhibition was estimated up to $46.8 \%$ in the preBötC glycinergic neurons by the optimized "EPF" method based on decay kinetics of inhibitory postsynaptic currents. The co-transmission in the preBötC was further corroborated by single cell RT-PCR that showed $64 \%$ glycinergic neurons co-express GABAergic neuronal markers. The drastically reduced VIAAT expression in the preBötC and hypoglossal nucleus of the conditional VIAAT KO embryos strongly suggest that glycinergic neuronal marker (GlyT2) is co-expressed in most of GABAergic neurons in embryonic brainstem. The complete absence of sIPSCs in embryonic hypoglossal motoneurons further confirms wide expression of cre-recombinase among inhibitory neurons in a brainstem region which innervates hypoglossal nucleus. These results strongly suggest that GABA and glycine co-transmission could be ubiquitously present in the embryonic respiratory network and its distribution and relevance decreases during development.

The cVIAAT KO embryos died due to respiratory failure resulting from complete loss of inhibitory transmission which is required for coupling between the preBötC and the pFRG to produce a normal respiratory rhythm. A disturbance in either glycine transmission or GABAergic transmission alone does not deteriorate the respiratory network immediately because the co-transmission of GABA and glycine provides a mechanism of compensation for loss of single inhibitory transmitter as gephyrin KO, GlyT2 KO and GAD67 mouse models survive longer than cVIAAT and VIAAT KO embryos. The CVIAAT KO embryos without any exception showed very common striking features like omphalocele, cleft palate and kyphotic posture which must be due to the loss of all inhibition but not with either GABAergic or glycinergic inhibition alone. 


\section{References}

Abdala AP, B.H., Rybak IA, Smith JC, Paton JF (2010) Role of GlyT2 expressing neurons in the Bo"tzinger complex for respiratory rhythm and pattern generation. . FASEB $J$ 24:614.3.

Abdala, A.P., Rybak, I.A., Smith, J.C., Zoccal, D.B., Machado, B.H., St-John, W.M. \& Paton, J.F. (2009) Multiple pontomedullary mechanisms of respiratory rhythmogenesis. Respir Physiol Neurobiol, 168, 19-25.

Adams, R.H., Sato, K., Shimada, S., Tohyama, M., Puschel, A.W. \& Betz, H. (1995) Gene structure and glial expression of the glycine transporter GlyT1 in embryonic and adult rodents. The Journal of neuroscience : the official journal of the Society for Neuroscience, 15, 2524-2532.

Aguayo, L.G., van Zundert, B., Tapia, J.C., Carrasco, M.A. \& Alvarez, F.J. (2004) Changes on the properties of glycine receptors during neuronal development. Brain Res Brain Res Rev, 47, 33-45.

Aldes, L.D., Chronister, R.B. \& Marco, L.A. (1988) Distribution of glutamic acid decarboxylase and gamma-aminobutyric acid in the hypoglossal nucleus in the rat. J Neurosci Res, 19, 343348.

Alheid, G.F. \& McCrimmon, D.R. (2008) The chemical neuroanatomy of breathing. Respir Physiol Neurobiol, 164, 3-11.

Amir, R.E., Van den Veyver, I.B., Wan, M., Tran, C.Q., Francke, U. \& Zoghbi, H.Y. (1999) Rett syndrome is caused by mutations in X-linked MECP2, encoding methyl-CpG-binding protein 2. Nat Genet, 23, 185-188.

Apostolides, P.F. \& Trussell, L.O. (2013) Rapid, Activity-Independent Turnover of Vesicular Transmitter Content at a Mixed Glycine/GABA Synapse. The Journal of Neuroscience, 33, 4768-4781.

Asada, H., Kawamura, Y., Maruyama, K., Kume, H., Ding, R., Ji, F.Y., Kanbara, N., Kuzume, H., Sanbo, M., Yagi, T. \& Obata, K. (1996) Mice lacking the $65 \mathrm{kDa}$ isoform of glutamic acid decarboxylase (GAD65) maintain normal levels of GAD67 and GABA in their brains but are susceptible to seizures. Biochem Biophys Res Commun, 229, 891-895.

Asada, H., Kawamura, Y., Maruyama, K., Kume, H., Ding, R.G., Kanbara, N., Kuzume, H., Sanbo, M., Yagi, T. \& Obata, K. (1997) Cleft palate and decreased brain gamma-aminobutyric acid in mice lacking the 67-kDa isoform of glutamic acid decarboxylase. Proc Natl Acad Sci U $S A, 94,6496-6499$.

Aubrey, K.R., Rossi, F.M., Ruivo, R., Alboni, S., Bellenchi, G.C., Le Goff, A., Gasnier, B. \& Supplisson, S. (2007) The Transporters GlyT2 and VIAAT Cooperate to Determine the Vesicular Glycinergic Phenotype. The Journal of Neuroscience, 27, 6273-6281. 
Awatramani, G.B., Turecek, R. \& Trussell, L.O. (2005) Staggered development of GABAergic and glycinergic transmission in the MNTB. J Neurophysiol, 93, 819-828.

Banks, G.B., Kanjhan, R., Wiese, S., Kneussel, M., Wong, L.M., O'Sullivan, G., Sendtner, M., Bellingham, M.C., Betz, H. \& Noakes, P.G. (2005) Glycinergic and GABAergic synaptic activity differentially regulate motoneuron survival and skeletal muscle innervation. $J$ Neurosci, 25, 1249-1259.

Belachew, S., Malgrange, B., Rigo, J.M., Rogister, B., Leprince, P., Hans, G., Nguyen, L. \& Moonen, G. (2000) Glycine triggers an intracellular calcium influx in oligodendrocyte progenitor cells which is mediated by the activation of both the ionotropic glycine receptor and Na+-dependent transporters. Eur J Neurosci, 12, 1924-1930.

Ben-Ari, Y. (2001) Developing networks play a similar melody. Trends Neurosci, 24, 353-360.

Ben-Ari, Y. (2002) Excitatory actions of gaba during development: the nature of the nurture. Nat Rev Neurosci, 3, 728-739.

Berger, A.J. (2011) Development of synaptic transmission to respiratory motoneurons. Respiratory physiology \& neurobiology, 179, 34-42.

Biel, M., Wahl-Schott, C., Michalakis, S. \& Zong, X. (2009) Hyperpolarization-activated cation channels: from genes to function. Physiol Rev, 89, 847-885.

Bongianni, F., Mutolo, D., Cinelli, E. \& Pantaleo, T. (2010) Respiratory responses induced by blockades of GABA and glycine receptors within the Botzinger complex and the preBotzinger complex of the rabbit. Brain Res, 1344, 134-147.

Borden, L.A., Smith, K.E., Vaysse, P.J., Gustafson, E.L., Weinshank, R.L. \& Branchek, T.A. (1995) Re-evaluation of GABA transport in neuronal and glial cell cultures: correlation of pharmacology and mRNA localization. Receptors Channels, 3, 129-146.

Borowsky, B., Mezey, E. \& Hoffman, B.J. (1993) Two glycine transporter variants with distinct localization in the CNS and peripheral tissues are encoded by a common gene. Neuron, 10, 851-863.

Boscan, P., Pickering, A.E. \& Paton, J.F. (2002) The nucleus of the solitary tract: an integrating station for nociceptive and cardiorespiratory afferents. Experimental Physiology, 87, 259266.

Bosma, P.T., Blazquez, M., Collins, M.A., Bishop, J.D., Drouin, G., Priede, I.G., Docherty, K. \& Trudeau, V.L. (1999) Multiplicity of glutamic acid decarboxylases (GAD) in vertebrates: molecular phylogeny and evidence for a new GAD paralog. Mol Biol Evol, 16, 397-404.

Bou-Flores, C. \& Berger, A.J. (2001) Gap junctions and inhibitory synapses modulate inspiratory motoneuron synchronization. J Neurophysiol, 85, 1543-1551. 
Bouvier, J., Thoby-Brisson, M., Renier, N., Dubreuil, V., Ericson, J., Champagnat, J., Pierani, A., Chedotal, A. \& Fortin, G. (2010) Hindbrain interneurons and axon guidance signaling critical for breathing. Nat Neurosci, 13, 1066-1074.

Burger, P.M., Hell, J., Mehl, E., Krasel, C., Lottspeich, F. \& Jahn, R. (1991) GABA and glycine in synaptic vesicles: storage and transport characteristics. Neuron, 7, 287-293.

Burkat, P.M., Yang, J. \& Gingrich, K.J. (2001) Dominant gating governing transient GABA(A) receptor activity: a first latency and Po/o analysis. The Journal of neuroscience : the official journal of the Society for Neuroscience, 21, 7026-7036.

Burnstock, G. (1976) Do some nerve cells release more than one transmitter? Neuroscience, 1, 239-248.

Busselberg, D., Bischoff, A.M., Paton, J.F. \& Richter, D.W. (2001) Reorganisation of respiratory network activity after loss of glycinergic inhibition. Pflugers Arch, 441, 444-449.

Chalphin, A.V. \& Saha, M.S. (2010) The specification of glycinergic neurons and the role of glycinergic transmission in development. Front Mol Neurosci, 3, 11.

Champagnat, J., Morin-Surun, M.P., Fortin, G. \& Thoby-Brisson, M. (2009) Developmental basis of the rostro-caudal organization of the brainstem respiratory rhythm generator. Philos Trans $R$ Soc Lond B Biol Sci, 364, 2469-2476.

Chaudhry, F.A., Reimer, R.J., Bellocchio, E.E., Danbolt, N.C., Osen, K.K., Edwards, R.H. \& Storm-Mathisen, J. (1998) The vesicular GABA transporter, VGAT, localizes to synaptic vesicles in sets of glycinergic as well as GABAergic neurons. J Neurosci, 18, 9733-9750.

Chen, M.L. \& Keens, T.G. (2004) Congenital central hypoventilation syndrome: not just another rare disorder. Paediatr Respir Rev, 5, 182-189.

Cherubini, E. \& Conti, F. (2001) Generating diversity at GABAergic synapses. Trends Neurosci, 24, 155-162.

Cherubini, E., Gaiarsa, J.L. \& Ben-Ari, Y. (1991) GABA: an excitatory transmitter in early postnatal life. Trends Neurosci, 14, 515-519.

Chessler, S.D., Simonson, W.T., Sweet, I.R. \& Hammerle, L.P. (2002) Expression of the vesicular inhibitory amino acid transporter in pancreatic islet cells: distribution of the transporter within rat islets. Diabetes, 51, 1763-1771.

Comer, A.M., Gibbons, H.M., Qi, J., Kawai, Y., Win, J. \& Lipski, J. (1999) Detection of mRNA species in bulbospinal neurons isolated from the rostral ventrolateral medulla using singlecell RT-PCR. Brain Res Brain Res Protoc, 4, 367-377.

Condie, B.G., Bain, G., Gottlieb, D.I. \& Capecchi, M.R. (1997) Cleft palate in mice with a targeted mutation in the gamma-aminobutyric acid-producing enzyme glutamic acid decarboxylase 67. Proc Natl Acad Sci U S A, 94, 11451-11455. 
Connors, B.W. \& Long, M.A. (2004) Electrical synapses in the mammalian brain. Annu Rev Neurosci, 27, 393-418.

Culiat, C.T., Stubbs, L.J., Woychik, R.P., Russell, L.B., Johnson, D.K. \& Rinchik, E.M. (1995) Deficiency of the beta 3 subunit of the type A gamma-aminobutyric acid receptor causes cleft palate in mice. Nat Genet, 11, 344-346.

Dale, H. (1935) Pharmacology and Nerve-endings (Walter Ernest Dixon Memorial Lecture): (Section of Therapeutics and Pharmacology). Proc R Soc Med, 28, 319-332.

Dale, H.H. (1914) The action of certain esters and ethers of choline, and thier relation to muscarine. Journal of Pharmacology and Experimental Therapeutics, 6, 147-190.

de Vries, J.I., Visser, G.H. \& Prechtl, H.F. (1982) The emergence of fetal behaviour. I. Qualitative aspects. Early Hum Dev, 7, 301-322.

Decavel, C. \& Van den Pol, A.N. (1990) GABA: a dominant neurotransmitter in the hypothalamus. The Journal of comparative neurology, 302, 1019-1037.

Del Negro, C.A., Koshiya, N., Butera, R.J., Jr. \& Smith, J.C. (2002a) Persistent sodium current, membrane properties and bursting behavior of pre-botzinger complex inspiratory neurons in vitro. J Neurophysiol, 88, 2242-2250.

Del Negro, C.A., Morgado-Valle, C. \& Feldman, J.L. (2002b) Respiratory rhythm: an emergent network property? Neuron, 34, 821-830.

den Eynden, J.V., Ali, S.S., Horwood, N., Carmans, S., Brone, B., Hellings, N., Steels, P., Harvey, R.J. \& Rigo, J.M. (2009) Glycine and glycine receptor signalling in non-neuronal cells. Front Mol Neurosci, 2, 9.

Dodt, H.U. \& Zieglgansberger, W. (1990) Infrared videomicroscopy: a new look at neuronal structure and function. Trends-Neurosci, 17, 453-458.

Dufour, A., Tell, F., Kessler, J.P. \& Baude, A. (2010) Mixed GABA-glycine synapses delineate a specific topography in the nucleus tractus solitarii of adult rat. J Physiol, 588, 1097-1115.

Dugue, G.P., Dumoulin, A., Triller, A. \& Dieudonne, S. (2005) Target-dependent use of coreleased inhibitory transmitters at central synapses. J Neurosci, 25, 6490-6498.

Dumoulin, A., Rostaing, P., Bedet, C., Levi, S., Isambert, M.F., Henry, J.P., Triller, A. \& Gasnier, B. (1999) Presence of the vesicular inhibitory amino acid transporter in GABAergic and glycinergic synaptic terminal boutons. J Cell Sci, 112 ( Pt 6), 811-823.

Dumoulin, A., Triller, A. \& Dieudonne, S. (2001) IPSC kinetics at identified GABAergic and mixed GABAergic and glycinergic synapses onto cerebellar Golgi cells. J Neurosci, 21, 60456057. 
Dutschmann, M. \& Herbert, H. (2006) The Kolliker-Fuse nucleus gates the postinspiratory phase of the respiratory cycle to control inspiratory off-switch and upper airway resistance in rat. Eur J Neurosci, 24, 1071-1084.

Ebihara, S., Obata, K. \& Yanagawa, Y. (2003) Mouse vesicular GABA transporter gene: genomic organization, transcriptional regulation and chromosomal localization. Brain Res Mol Brain Res, 110, 126-139.

Ezure, K., Manabe, M. \& Yamada, H. (1988) Distribution of medullary respiratory neurons in the rat. Brain Res, 455, 262-270.

Ezure, K., Tanaka, I. \& Kondo, M. (2003a) Glycine is used as a transmitter by decrementing expiratory neurons of the ventrolateral medulla in the rat. $J$ Neurosci, 23, 8941-8948.

Ezure, K., Tanaka, I. \& Saito, Y. (2003b) Brainstem and spinal projections of augmenting expiratory neurons in the rat. Neurosci Res, 45, 41-51.

Farrar, S.J., Whiting, P.J., Bonnert, T.P. \& McKernan, R.M. (1999) Stoichiometry of a ligandgated ion channel determined by fluorescence energy transfer. J Biol Chem, 274, 1010010104.

Feldman, J.L. \& Del Negro, C.A. (2006) Looking for inspiration: new perspectives on respiratory rhythm. Nat Rev Neurosci, 7, 232-242.

Feldman, J.L., Mitchell, G.S. \& Nattie, E.E. (2003) Breathing: rhythmicity, plasticity, chemosensitivity. Annu Rev Neurosci, 26, 239-266.

Feng, G., Tintrup, H., Kirsch, J., Nichol, M.C., Kuhse, J., Betz, H. \& Sanes, J.R. (1998) Dual requirement for gephyrin in glycine receptor clustering and molybdoenzyme activity. Science, 282, 1321-1324.

Flint, A.C., Liu, X. \& Kriegstein, A.R. (1998) Nonsynaptic glycine receptor activation during early neocortical development. Neuron, 20, 43-53.

Fogarty, M.J., Smallcombe, K.L., Yanagawa, Y., Obata, K., Bellingham, M.C. \& Noakes, P.G. (2013a) Genetic Deficiency of GABA Differentially Regulates Respiratory and Non-Respiratory Motor Neuron Development. PLoS One, 8, e56257.

Fogarty, M.J., Yanagawa, Y., Obata, K., Bellingham, M.C. \& Noakes, P.G. (2013b) Genetic absence of the vesicular inhibitory amino acid transporter differentially regulates respiratory and locomotor motor neuron development. Brain structure \& function.

Fortin, G. \& Thoby-Brisson, M. (2009) Embryonic emergence of the respiratory rhythm generator. Respir Physiol Neurobiol, 168, 86-91.

Freichel, C., Potschka, H., Ebert, U., Brandt, C. \& Loscher, W. (2006) Acute changes in the neuronal expression of GABA and glutamate decarboxylase isoforms in the rat piriform cortex following status epilepticus. Neuroscience, 141, 2177-2194. 
Fritschy, J.M., Paysan, J., Enna, A. \& Mohler, H. (1994) Switch in the expression of rat GABAAreceptor subtypes during postnatal development: an immunohistochemical study. J Neurosci, 14, 5302-5324.

Fujii, M., Arata, A., Kanbara-Kume, N., Saito, K., Yanagawa, Y. \& Obata, K. (2007) Respiratory activity in brainstem of fetal mice lacking glutamate decarboxylase 65/67 and vesicular GABA transporter. Neuroscience, 146, 1044-1052.

Furshpan, E.J., MacLeish, P.R., O'Lague, P.H. \& Potter, D.D. (1976) Chemical transmission between rat sympathetic neurons and cardiac myocytes developing in microcultures: evidence for cholinergic, adrenergic, and dual-function neurons. Proc Natl Acad Sci U S A, 73, 4225-4229.

Gaiarsa, J.L., Caillard, O. \& Ben-Ari, Y. (2002) Long-term plasticity at GABAergic and glycinergic synapses: mechanisms and functional significance. Trends Neurosci, 25, 564-570.

Gammelsaeter, R., Froyland, M., Aragon, C., Danbolt, N.C., Fortin, D., Storm-Mathisen, J., Davanger, S. \& Gundersen, V. (2004) Glycine, GABA and their transporters in pancreatic islets of Langerhans: evidence for a paracrine transmitter interplay. J Cell Sci, 117, 37493758.

Gao, B.X., Stricker, C. \& Ziskind-Conhaim, L. (2001) Transition from GABAergic to glycinergic synaptic transmission in newly formed spinal networks. J Neurophysiol, 86, 492-502.

Gao, B.X. \& Ziskind-Conhaim, L. (1995) Development of glycine- and GABA-gated currents in rat spinal motoneurons. J Neurophysiol, 74, 113-121.

Garcia, A.J., 3rd, Koschnitzky, J.E. \& Ramirez, J.M. (2013) The Physiological Determinants of Sudden Infant Death Syndrome. Respir Physiol Neurobiol.

Gomeza, J., Hulsmann, S., Ohno, K., Eulenburg, V., Szoke, K., Richter, D. \& Betz, H. (2003a) Inactivation of the glycine transporter 1 gene discloses vital role of glial glycine uptake in glycinergic inhibition. Neuron, 40, 785-796.

Gomeza, J., Ohno, K., Hulsmann, S., Armsen, W., Eulenburg, V., Richter, D.W., Laube, B. \& Betz, H. (2003b) Deletion of the mouse glycine transporter 2 results in a hyperekplexia phenotype and postnatal lethality. Neuron, 40, 797-806.

Goulon, M. \& Goulon-Goeau, C. (1989) Amyotrophic lateral sclerosis and respiratory assistance. Rev Neurol (Paris), 145, 293-298.

Gozal, D. (2004) New concepts in abnormalities of respiratory control in children. Curr Opin Pediatr, 16, 305-308.

Graham, L.T., Jr., Shank, R.P., Werman, R. \& Aprison, M.H. (1967) Distribution of some synaptic transmitter suspects in cat spinal cord: glutamic acid, aspartic acid, gammaaminobutyric acid, glycine and glutamine. J Neurochem, 14, 465-472. 
Gray, P.A., Hayes, J.A., Ling, G.Y., Llona, I., Tupal, S., Picardo, M.C., Ross, S.E., Hirata, T., Corbin, J.G., Eugenin, J. \& Del Negro, C.A. (2010) Developmental origin of preBotzinger complex respiratory neurons. J Neurosci, 30, 14883-14895.

Gray, P.A., Janczewski, W.A., Mellen, N., McCrimmon, D.R. \& Feldman, J.L. (2001) Normal breathing requires preBotzinger complex neurokinin-1 receptor-expressing neurons. Nat Neurosci, 4, 927-930.

Gray, P.A., Rekling, J.C., Bocchiaro, C.M. \& Feldman, J.L. (1999) Modulation of Respiratory Frequency by Peptidergic Input to Rhythmogenic Neurons in the PreBötzinger Complex. Science, 286, 1566-1568.

Greer, J.J. \& Funk, G.D. (2005) Perinatal development of respiratory motoneurons. Respir Physiol Neurobiol, 149, 43-61.

Guyenet, P.G. \& Wang, H. (2001) Pre-Bötzinger Neurons With Preinspiratory Discharges "In Vivo" Express NK1 Receptors in the Rat. Journal of Neurophysiology, 86, 438-446.

Hayashi, F. \& Lipski, J. (1992) The role of inhibitory amino acids in control of respiratory motor output in an arterially perfused rat. Respir Physiol, 89, 47-63.

Hernandes, M.S. \& Troncone, L.R. (2009) Glycine as a neurotransmitter in the forebrain: a short review. J Neural Transm, 116, 1551-1560.

Hoch, W., Betz, H. \& Becker, C.M. (1989) Primary cultures of mouse spinal cord express the neonatal isoform of the inhibitory glycine receptor. Neuron, 3, 339-348.

Horner, R.L. (2007) Respiratory motor activity: influence of neuromodulators and implications for sleep disordered breathing. Can J Physiol Pharmacol, 85, 155-165.

Hubner, C.A., Stein, V., Hermans-Borgmeyer, I., Meyer, T., Ballanyi, K. \& Jentsch, T.J. (2001) Disruption of $\mathrm{KCC} 2$ reveals an essential role of $\mathrm{K}-\mathrm{Cl}$ cotransport already in early synaptic inhibition. Neuron, 30, 515-524.

Hulsmann, S., Oku, Y., Zhang, W. \& Richter, D.W. (2000) Metabotropic glutamate receptors and blockade of glial Krebs cycle depress glycinergic synaptic currents of mouse hypoglossal motoneurons. The European journal of neuroscience, 12, 239-246.

Ikeda, K., Onimaru, H., Yamada, J., Inoue, K., Ueno, S., Onaka, T., Toyoda, H., Arata, A., Ishikawa, T.O., Taketo, M.M., Fukuda, A. \& Kawakami, K. (2004) Malfunction of respiratoryrelated neuronal activity in $\mathrm{Na}+\mathrm{K}+-\mathrm{ATPase}$ alpha2 subunit-deficient mice is attributable to abnormal Cl- homeostasis in brainstem neurons. J Neurosci, 24, 10693-10701.

Ishibashi, H., Yamaguchi, J., Nakahata, Y. \& Nabekura, J. (2013) Dynamic regulation of glycine/GABA co-transmission at spinal inhibitory synapses by neuronal glutamate transporter. The Journal of Physiology. 
Ishihara, N., Armsen, W., Papadopoulos, T., Betz, H. \& Eulenburg, V. (2010) Generation of a mouse line expressing Cre recombinase in glycinergic interneurons. Genesis, 48, 437-445.

Janczewski, W.A. \& Feldman, J.L. (2006a) Distinct rhythm generators for inspiration and expiration in the juvenile rat. J Physiol, 570, 407-420.

Janczewski, W.A. \& Feldman, J.L. (2006b) Novel data supporting the two respiratory rhythm oscillator hypothesis. Focus on "respiration-related rhythmic activity in the rostral medulla of newborn rats". J Neurophysiol, 96, 1-2.

Janczewski, W.A., Tashima, A., Hsu, P., Cui, Y. \& Feldman, J.L. (2013) Role of Inhibition in Respiratory Pattern Generation. The Journal of Neuroscience, 33, 5454-5465.

Jensen, K., Chiu, C.S., Sokolova, I., Lester, H.A. \& Mody, I. (2003) GABA transporter-1 (GAT1)deficient mice: differential tonic activation of GABAA versus GABAB receptors in the hippocampus. J Neurophysiol, 90, 2690-2701.

Jonas, P., Bischofberger, J. \& Sandkuhler, J. (1998) Corelease of two fast neurotransmitters at a central synapse. Science, 281, 419-424.

Jursky, F. \& Nelson, N. (1996) Developmental expression of the glycine transporters GLYT1 and GLYT2 in mouse brain. $J$ Neurochem, 67, 336-344.

Kiehn, O. \& Tresch, M.C. (2002) Gap junctions and motor behavior. Trends Neurosci, 25, 108115.

Kirsch, J. \& Betz, H. (1998) Glycine-receptor activation is required for receptor clustering in spinal neurons. Nature, 392, 717-720.

Kneussel, M. \& Betz, H. (2000) Receptors, gephyrin and gephyrin-associated proteins: novel insights into the assembly of inhibitory postsynaptic membrane specializations. J Physiol, $\mathbf{5 2 5}$ Pt 1, 1-9.

Koizumi, H., Koshiya, N., Chia, J.X., Cao, F., Nugent, J., Zhang, R. \& Smith, J.C. (2013) Structural-Functional Properties of Identified Excitatory and Inhibitory Interneurons within Pre-BÃ $q$ tzinger Complex Respiratory Microcircuits. The Journal of Neuroscience, 33, 29943009.

Koizumi, H., Wilson, C.G., Wong, S., Yamanishi, T., Koshiya, N. \& Smith, J.C. (2008) Functional imaging, spatial reconstruction, and biophysical analysis of a respiratory motor circuit isolated in vitro. J Neurosci, 28, 2353-2365.

Kosel, M., Rudolph, U., Wielepp, P., Luginbuhl, M., Schmitt, W., Fisch, H.U. \& Schlaepfer, T.E. (2004) Diminished GABA(A) receptor-binding capacity and a DNA base substitution in a patient with treatment-resistant depression and anxiety. Neuropsychopharmacology, 29, 347-350. 
Kotak, V.C., Korada, S., Schwartz, I.R. \& Sanes, D.H. (1998) A developmental shift from GABAergic to glycinergic transmission in the central auditory system. J Neurosci, 18, 46464655.

Kuwana, S., Okada, Y., Sugawara, Y., Tsunekawa, N. \& Obata, K. (2003) Disturbance of neural respiratory control in neonatal mice lacking GABA synthesizing enzyme 67-kDa isoform of glutamic acid decarboxylase. Neuroscience, 120, 861-870.

Kuwana, S., Tsunekawa, N., Yanagawa, Y., Okada, Y., Kuribayashi, J. \& Obata, K. (2006) Electrophysiological and morphological characteristics of GABAergic respiratory neurons in the mouse pre-Botzinger complex. Eur J Neurosci, 23, 667-674.

Lal, A., Oku, Y., Hulsmann, S., Okada, Y., Miwakeichi, F., Kawai, S., Tamura, Y. \& Ishiguro, M. (2011) Dual oscillator model of the respiratory neuronal network generating quantal slowing of respiratory rhythm. J Comput Neurosci.

Langosch, D., Thomas, L. \& Betz, H. (1988) Conserved quaternary structure of ligand-gated ion channels: the postsynaptic glycine receptor is a pentamer. Proc Natl Acad Sci U S A, 85, 7394-7398.

Lara, J.P., Parkes, M.J., Silva-Carvhalo, L., Izzo, P., Dawid-Milner, M.S. \& Spyer, K.M. (1994) Cardiovascular and respiratory effects of stimulation of cell bodies of the parabrachial nuclei in the anaesthetized rat. J Physiol, 477 ( Pt 2), 321-329.

Latal, A.T., Kremer, T., Gomeza, J., Eulenburg, V. \& Hulsmann, S. (2010) Development of synaptic inhibition in glycine transporter 2 deficient mice. Mol Cell Neurosci, 44, 342-352.

Legendre, P. (2001) The glycinergic inhibitory synapse. Cell Mol Life Sci, 58, 760-793.

Lernmark, A. (1996) Glutamic acid decarboxylase--gene to antigen to disease. J Intern Med, 240, 259-277.

Levi, S., Vannier, C. \& Triller, A. (1998) Strychnine-sensitive stabilization of postsynaptic glycine receptor clusters. J Cell Sci, 111 ( Pt 3), 335-345.

Lindsey, B.G., Rybak, I.A. \& Smith, J.C. (2012) Computational models and emergent properties of respiratory neural networks. Compr Physiol, 2, 1619-1670.

Liu, Q. \& Wong-Riley, M.T. (2004) Developmental changes in the expression of GABAA receptor subunits alpha1, alpha2, and alpha3 in the rat pre-Botzinger complex. J Appl Physiol (1985), 96, 1825-1831.

Lowe, A.A. (1980) The neural regulation of tongue movements. Prog Neurobiol, 15, 295-344.

Lu, T., Rubio, M.E. \& Trussell, L.O. (2008) Glycinergic transmission shaped by the corelease of GABA in a mammalian auditory synapse. Neuron, 57, 524-535. 
Lynch, J.W. (2004) Molecular structure and function of the glycine receptor chloride channel. Physiol Rev, 84, 1051-1095.

Lynch, J.W. (2009) Native glycine receptor subtypes and their physiological roles. Neuropharmacology, 56, 303-309.

Maclntosh, D.J. (1977) Respiratory dysfunction in Parkinson's disease. Prim Care, 4, 441-445.

Martin-Caraballo, M. \& Greer, J.J. (1999) Electrophysiological properties of rat phrenic motoneurons during perinatal development. Journal of Neurophysiology, 81, 1365-1378.

McDonald, W.I. (1974) Pathophysiology in multiple sclerosis. Brain, 97, 179-196.

McIntire, S.L., Reimer, R.J., Schuske, K., Edwards, R.H. \& Jorgensen, E.M. (1997) Identification and characterization of the vesicular GABA transporter. Nature, 389, 870-876.

Mogami, H., Yura, S., Tatsumi, K., Fujii, T., Fujita, K., Kakui, K., Kondoh, E., Inoue, T., Fujii, S., Yodoi, J. \& Konishi, I. (2010) Thioredoxin binding protein-2 inhibits excessive fetal hypoglycemia during maternal starvation by suppressing insulin secretion in mice. Pediatr Res, 67, 138-143.

Morgado-Valle, C., Baca, S.M. \& Feldman, J.L. (2010) Glycinergic pacemaker neurons in preBotzinger complex of neonatal mouse. J Neurosci, 30, 3634-3639.

$\mathrm{Mu}$, L. \& Sanders, I. (2010) Human tongue neuroanatomy: Nerve supply and motor endplates. Clin Anat, 23, 777-791.

Nabekura, J., Katsurabayashi, S., Kakazu, Y., Shibata, S., Matsubara, A., Jinno, S., Mizoguchi, Y., Sasaki, A. \& Ishibashi, H. (2004) Developmental switch from GABA to glycine release in single central synaptic terminals. Nat Neurosci, 7, 17-23.

O'Brien, J.A. \& Berger, A.J. (1999) Cotransmission of GABA and glycine to brain stem motoneurons. J Neurophysiol, 82, 1638-1641.

Oh, W.J., Westmoreland, J.J., Summers, R. \& Condie, B.G. (2010) Cleft palate is caused by CNS dysfunction in Gad1 and Viaat knockout mice. PLoS One, 5, e9758.

Onimaru, H., Arata, A. \& Homma, I. (1990) Inhibitory synaptic inputs to the respiratory rhythm generator in the medulla isolated from newborn rats. Pflugers Archiv : European journal of physiology, 417, 425-432.

Onimaru, H. \& Homma, I. (2003) A novel functional neuron group for respiratory rhythm generation in the ventral medulla. J Neurosci, 23, 1478-1486.

Owman, C. (1964) Sympathetic Nerves Probably Storing Two Types of Monoamines in the Rat Pineal Gland. Int J Neuropharmacol, 3, 105-112. 
Pagliardini, S., Janczewski, W.A., Tan, W., Dickson, C.T., Deisseroth, K. \& Feldman, J.L. (2011) Active expiration induced by excitation of ventral medulla in adult anesthetized rats. J Neurosci, 31, 2895-2905.

Paton, J.F., Ramirez, J.M. \& Richter, D.W. (1994) Functionally intact in vitro preparation generating respiratory activity in neonatal and mature mammals. Pflugers Archiv : European journal of physiology, 428, 250-260.

Patterson, P.H. \& Chun, L.L. (1974) The influence of non-neuronal cells on catecholamine and acetylcholine synthesis and accumulation in cultures of dissociated sympathetic neurons. Proc Natl Acad Sci U S A, 71, 3607-3610.

Perrais, D. \& Ropert, N. (1999) Effect of zolpidem on miniature IPSCs and occupancy of postsynaptic GABAA receptors in central synapses. J Neurosci, 19, 578-588.

Plotkin, M.D., Snyder, E.Y., Hebert, S.C. \& Delpire, E. (1997) Expression of the Na-K-2Cl cotransporter is developmentally regulated in postnatal rat brains: a possible mechanism underlying GABA's excitatory role in immature brain. J Neurobiol, 33, 781-795.

Poh, C.H. (2013) The role of innervation during mouse embryonic myogenesis: what molecular genetics tells PhD thesis, pp. 192.

Rahman, J., Latal, A.T., Besser, S., Hirrlinger, J. \& Hulsmann, S. (2013) Mixed miniature postsynaptic currents resulting from co-release of glycine and GABA recorded from glycinergic neurons in the neonatal respiratory network. Eur J Neurosci.

Ramirez, J.M., Koch, H., Garcia, A.J., 3rd, Doi, A. \& Zanella, S. (2011) The role of spiking and bursting pacemakers in the neuronal control of breathing. J Biol Phys, 37, 241-261.

Ramirez, J.M., Quellmalz, U.J. \& Richter, D.W. (1996) Postnatal changes in the mammalian respiratory network as revealed by the transverse brainstem slice of mice. J Physiol, 491 ( Pt 3), 799-812.

Ramirez, J.M., Schwarzacher, S.W., Pierrefiche, O., Olivera, B.M. \& Richter, D.W. (1998) Selective lesioning of the cat pre-Botzinger complex in vivo eliminates breathing but not gasping. J Physiol, 507 ( Pt 3), 895-907.

Rekling, J.C., Champagnat, J. \& Denavit-Saubie, M. (1996) Electroresponsive properties and membrane potential trajectories of three types of inspiratory neurons in the newborn mouse brain stem in vitro. J Neurophysiol, 75, 795-810.

Rekling, J.C., Shao, X.M. \& Feldman, J.L. (2000) Electrical coupling and excitatory synaptic transmission between rhythmogenic respiratory neurons in the preBotzinger complex. $J$ Neurosci, 20, RC113.

Ren, J. \& Greer, J.J. (2006) Neurosteroid modulation of respiratory rhythm in rats during the perinatal period. J Physiol, 574, 535-546. 
Rhee, J.W., Arata, A., Selleri, L., Jacobs, Y., Arata, S., Onimaru, H. \& Cleary, M.L. (2004) Pbx3 deficiency results in central hypoventilation. Am J Pathol, 165, 1343-1350.

Rivera, C., Li, H., Thomas-Crusells, J., Lahtinen, H., Viitanen, T., Nanobashvili, A., Kokaia, Z., Airaksinen, M.S., Voipio, J., Kaila, K. \& Saarma, M. (2002) BDNF-induced TrkB activation down-regulates the $\mathrm{K}+-\mathrm{Cl}$ - cotransporter $\mathrm{KCC} 2$ and impairs neuronal $\mathrm{Cl}$ - extrusion. $J$ Cell Biol, 159, 747-752.

Rivera, C., Voipio, J., Payne, J.A., Ruusuvuori, E., Lahtinen, H., Lamsa, K., Pirvola, U., Saarma, M. \& Kaila, K. (1999) The K+/Cl- co-transporter KCC2 renders GABA hyperpolarizing during neuronal maturation. Nature, 397, 251-255.

Rosenmund, C. \& Stevens, C.F. (1996) Definition of the readily releasable pool of vesicles at hippocampal synapses. Neuron, 16, 1197-1207.

Russier, M., Kopysova, I.L., Ankri, N., Ferrand, N. \& Debanne, D. (2002) GABA and glycine corelease optimizes functional inhibition in rat brainstem motoneurons in vitro. $J$ Physiol, 541, 123-137.

Rybak, I.A., Abdala, A.P., Markin, S.N., Paton, J.F. \& Smith, J.C. (2007) Spatial organization and state-dependent mechanisms for respiratory rhythm and pattern generation. Prog Brain Res, 165, 201-220.

Sagne, C., El Mestikawy, S., Isambert, M.F., Hamon, M., Henry, J.P., Giros, B. \& Gasnier, B. (1997) Cloning of a functional vesicular GABA and glycine transporter by screening of genome databases. FEBS Lett, 417, 177-183.

Saito, K., Kakizaki, T., Hayashi, R., Nishimaru, H., Furukawa, T., Nakazato, Y., Takamori, S., Ebihara, S., Uematsu, M., Mishina, M., Miyazaki, J., Yokoyama, M., Konishi, S., Inoue, K., Fukuda, A., Fukumoto, M., Nakamura, K., Obata, K. \& Yanagawa, Y. (2010) The physiological roles of vesicular GABA transporter during embryonic development: a study using knockout mice. Mol Brain, 3, 40.

Sassoe-Pognetto, M., Kirsch, J., Grunert, U., Greferath, U., Fritschy, J.M., Mohler, H., Betz, H. \& Wassle, H. (1995) Colocalization of gephyrin and GABAA-receptor subunits in the rat retina. The Journal of comparative neurology, 357, 1-14.

Sato, K., Zhang, J.H., Saika, T., Sato, M., Tada, K. \& Tohyama, M. (1991) Localization of glycine receptor alpha 1 subunit mRNA-containing neurons in the rat brain: an analysis using in situ hybridization histochemistry. Neuroscience, 43, 381-395.

Schwarzacher, S.W., Smith, J.C. \& Richter, D.W. (1995) Pre-Botzinger complex in the cat. Journal of Neurophysiology, 73, 1452-1461.

Sebe, J.Y., van Brederode, J.F. \& Berger, A.J. (2006) Inhibitory synaptic transmission governs inspiratory motoneuron synchronization. Journal of Neurophysiology, 96, 391-403. 
Semyanov, A., Walker, M.C. \& Kullmann, D.M. (2003) GABA uptake regulates cortical excitability via cell type-specific tonic inhibition. Nat Neurosci, 6, 484-490.

Shao, X.M. \& Feldman, J.L. (1997) Respiratory rhythm generation and synaptic inhibition of expiratory neurons in pre-Botzinger complex: differential roles of glycinergic and GABAergic neural transmission. J Neurophysiol, 77, 1853-1860.

Shino, M., Kaneko, R., Yanagawa, Y., Kawaguchi, Y. \& Saito, Y. (2011) Electrophysiological characteristics of inhibitory neurons of the prepositus hypoglossi nucleus as analyzed in Venus-expressing transgenic rats. Neuroscience, 197, 89-98.

Shino, M., Ozawa, S., Furuya, N. \& Saito, Y. (2008) Membrane properties of excitatory and inhibitory neurons in the rat prepositus hypoglossi nucleus. Eur J Neurosci, 27, 2413-2424.

Simat, M., Ambrosetti, L., Lardi-Studler, B. \& Fritschy, J.M. (2007a) GABAergic synaptogenesis marks the onset of differentiation of basket and stellate cells in mouse cerebellum. Eur J Neurosci, 26, 2239-2256.

Simat, M., Parpan, F. \& Fritschy, J.M. (2007b) Heterogeneity of glycinergic and gabaergic interneurons in the granule cell layer of mouse cerebellum. J Comp Neurol, 500, 71-83.

Singer, J.H. (2008) GABA is an endogenous ligand for synaptic glycine receptors. Neuron, 57, 475-477.

Singer, J.H. \& Berger, A.J. (2000) Development of inhibitory synaptic transmission to motoneurons. Brain Res Bull, 53, 553-560.

Smith, J.C., Abdala, A.P., Borgmann, A., Rybak, I.A. \& Paton, J.F. (2013) Brainstem respiratory networks: building blocks and microcircuits. Trends Neurosci, 36, 152-162.

Smith, J.C., Abdala, A.P., Koizumi, H., Rybak, I.A. \& Paton, J.F. (2007) Spatial and functional architecture of the mammalian brain stem respiratory network: a hierarchy of three oscillatory mechanisms. J Neurophysiol, 98, 3370-3387.

Smith, J.C., Ellenberger, H.H., Ballanyi, K., Richter, D.W. \& Feldman, J.L. (1991) Pre-Botzinger complex: a brainstem region that may generate respiratory rhythm in mammals. Science, 254, 726-729.

Smith, S.M., Zorec, R. \& McBurney, R.N. (1989) Conductance states activated by glycine and GABA in rat cultured spinal neurones. J Membr Biol, 108, 45-52.

Sokoloff, A.J. \& Deacon, T.W. (1992) Musculotopic organization of the hypoglossal nucleus in the cynomolgus monkey, Macaca fascicularis. The Journal of Comparative Neurology, 324, 81-93.

Spyer, K.M. (2009) To breathe or not to breathe? That is the question. Experimental Physiology, 94, 1-10. 
Stornetta, R.L., Rosin, D.L., Wang, H., Sevigny, C.P., Weston, M.C. \& Guyenet, P.G. (2003) A group of glutamatergic interneurons expressing high levels of both neurokinin-1 receptors and somatostatin identifies the region of the pre-Botzinger complex. The Journal of comparative neurology, 455, 499-512.

Supplisson, S. \& Roux, M.J. (2002) Why glycine transporters have different stoichiometries. FEBS Lett, 529, 93-101.

Szabo, G., Katarova, Z. \& Greenspan, R. (1994) Distinct protein forms are produced from alternatively spliced bicistronic glutamic acid decarboxylase mRNAs during development. Mol Cell Biol, 14, 7535-7545.

Szoke, K., Hartel, K., Grass, D., Hirrlinger, P.G., Hirrlinger, J. \& Hulsmann, S. (2006) Glycine transporter 1 expression in the ventral respiratory group is restricted to protoplasmic astrocytes. Brain Res, 1119, 182-189.

Tan, W., Janczewski, W.A., Yang, P., Shao, X.M., Callaway, E.M. \& Feldman, J.L. (2008) Silencing preBotzinger complex somatostatin-expressing neurons induces persistent apnea in awake rat. Nat Neurosci, 11, 538-540.

Tan, W., Pagliardini, S., Yang, P., Janczewski, W.A. \& Feldman, J.L. (2010) Projections of preBotzinger complex neurons in adult rats. J Comp Neurol, 518, 1862-1878.

Tanaka, I. \& Ezure, K. (2004) Overall distribution of GLYT2 mRNA-containing versus GAD67 mRNA-containing neurons and colocalization of both mRNAs in midbrain, pons, and cerebellum in rats. Neurosci Res, 49, 165-178.

Taneera, J., Jin, Z., Jin, Y., Muhammed, S.J., Zhang, E., Lang, S., Salehi, A., Korsgren, O., Renstrom, E., Groop, L. \& Birnir, B. (2012) gamma-Aminobutyric acid (GABA) signalling in human pancreatic islets is altered in type 2 diabetes. Diabetologia, 55, 1985-1994.

Tatetsu, M., Kim, J., Kina, S., Sunakawa, H. \& Takayama, C. (2012) GABA/glycine signaling during degeneration and regeneration of mouse hypoglossal nerves. Brain Res, 1446, 22-33.

Thoby-Brisson, M., Karlen, M., Wu, N., Charnay, P., Champagnat, J. \& Fortin, G. (2009) Genetic identification of an embryonic parafacial oscillator coupling to the preBotzinger complex. Nat Neurosci, 12, 1028-1035.

Thoby-Brisson, M., Trinh, J.B., Champagnat, J. \& Fortin, G. (2005) Emergence of the preBotzinger respiratory rhythm generator in the mouse embryo. J Neurosci, 25, 4307-4318.

Tian, G.F., Peever, J.H. \& Duffin, J. (1998) Botzinger-complex expiratory neurons monosynaptically inhibit phrenic motoneurons in the decerebrate rat. Exp Brain Res, 122, 149-156.

Todd, A.J., Watt, C., Spike, R.C. \& Sieghart, W. (1996) Colocalization of GABA, glycine, and their receptors at synapses in the rat spinal cord. J Neurosci, 16, 974-982. 
Tsunekawa, N., Arata, A. \& Obata, K. (2005) Development of spontaneous mouth/tongue movement and related neural activity, and their repression in fetal mice lacking glutamate decarboxylase 67. Eur J Neurosci, 21, 173-178.

Twyman, R.E. \& Macdonald, R.L. (1991) Kinetic properties of the glycine receptor main- and sub-conductance states of mouse spinal cord neurones in culture. The Journal of Physiology, 435, 303-331.

van Brederode, J.F., Yanagawa, Y. \& Berger, A.J. (2011) GAD67-GFP+ neurons in the Nucleus of Roller: a possible source of inhibitory input to hypoglossal motoneurons. I. Morphology and firing properties. J Neurophysiol, 105, 235-248.

Viemari, J.C., Burnet, H., Bevengut, M. \& Hilaire, G. (2003) Perinatal maturation of the mouse respiratory rhythm-generator: in vivo and in vitro studies. Eur J Neurosci, 17, 1233-1244.

Wallen-Mackenzie, A., Gezelius, H., Thoby-Brisson, M., Nygard, A., Enjin, A., Fujiyama, F., Fortin, G. \& Kullander, K. (2006a) Vesicular glutamate transporter 2 is required for central respiratory rhythm generation but not for locomotor central pattern generation. The Journal of neuroscience : the official journal of the Society for Neuroscience, 26, 12294-12307.

Wallen-Mackenzie, A., Gezelius, H., Thoby-Brisson, M., Nygard, A., Enjin, A., Fujiyama, F., Fortin, G. \& Kullander, K. (2006b) Vesicular glutamate transporter 2 is required for central respiratory rhythm generation but not for locomotor central pattern generation. $J$ Neurosci, 26, 12294-12307.

Wang, H., Stornetta, R.L., Rosin, D.L. \& Guyenet, P.G. (2001) Neurokinin-1 receptorimmunoreactive neurons of the ventral respiratory group in the rat. The Journal of comparative neurology, 434, 128-146.

Weese-Mayer, D.E., Zhou, L., Berry-Kravis, E.M., Maher, B.S., Silvestri, J.M. \& Marazita, M.L. (2003) Association of the serotonin transporter gene with sudden infant death syndrome: a haplotype analysis. Am J Med Genet A, 122A, 238-245.

White, W.F., O'Gorman, S. \& Roe, A.W. (1990) Three-dimensional autoradiographic localization of quench-corrected glycine receptor specific activity in the mouse brain using 3H-strychnine as the ligand. J Neurosci, 10, 795-813.

Whittaker, V.P., Dowdall, M.J. \& Boyne, A.F. (1972) The storage and release of acetylcholine by cholinergic nerve terminals: recent results with non-mammalian preparations. Biochem Soc Symp, 49-68.

Winter, S.M., Fresemann, J., Schnell, C., Oku, Y., Hirrlinger, J. \& Hulsmann, S. (2009) Glycinergic interneurons are functionally integrated into the inspiratory network of mouse medullary slices. Pflugers Arch, 458, 459-469.

Winter, S.M., Fresemann, J., Schnell, C., Oku, Y., Hirrlinger, J. \& Hulsmann, S. (2010) Glycinergic interneurons in the respiratory network of the rhythmic slice preparation. $A d v$ Exp Med Biol, 669, 97-100. 
Wittmeier, S., Song, G., Duffin, J. \& Poon, C.S. (2008) Pacemakers handshake synchronization mechanism of mammalian respiratory rhythmogenesis. Proc Natl Acad Sci U S A, 105, 1800018005.

Wojcik, S.M., Katsurabayashi, S., Guillemin, I., Friauf, E., Rosenmund, C., Brose, N. \& Rhee, J.S. (2006) A shared vesicular carrier allows synaptic corelease of GABA and glycine. Neuron, 50, 575-587.

Yamanaka, I., Miki, M., Asakawa, K., Kawakami, K., Oda, Y. \& Hirata, H. (2013) Glycinergic transmission and postsynaptic activation of CaMKII are required for glycine receptor clustering in vivo. Genes Cells.

Yamuy, J., Engelhardt, J.K., Morales, F.R. \& Chase, M.H. (1992) Passive electrical properties of motoneurons in aged cats following axotomy. Brain research, 570, 300-306.

Yazulla, S. \& Yang, C.Y. (1988) Colocalization of GABA and glycine immunoreactivities in a subset of retinal neurons in tiger salamander. Neurosci Lett, 95, 37-41.

Zafra, F., Aragon, C., Olivares, L., Danbolt, N.C., Gimenez, C. \& Storm-Mathisen, J. (1995) Glycine transporters are differentially expressed among CNS cells. J Neurosci, 15, 3952-3969.

Zeilhofer, H.U., Studler, B., Arabadzisz, D., Schweizer, C., Ahmadi, S., Layh, B., Bosl, M.R. \& Fritschy, J.M. (2005) Glycinergic neurons expressing enhanced green fluorescent protein in bacterial artificial chromosome transgenic mice. J Comp Neurol, 482, 123-141. 


\section{List of publications}

Rahman, J., Latal, A.T., Besser, S., Hirrlinger, J. \& Hulsmann, S. (2013) Mixed miniature postsynaptic currents resulting from co-release of glycine and GABA recorded from glycinergic neurons in the neonatal respiratory network. Eur J Neurosci.

Another manuscript titled "Genetic ablation of VIAAT expression in glycinergic neurons causes severe respiratory phenotype and perinatal death" is about to submit. 


\section{Acknowledgements}

First I would like to thank my supervisor Prof. Swen Hülsmann for accepting me into his group to work on this excellent project. Most importantly, I thank him for scientific trainings and regular discussions which he provided from beginning of the thesis that helped me to advance in research career. I also extend thank to his kind diligence beyond science.

I would also like to express my gratitude to thesis committee members, Prof. Ralf Heinrich and Dr. Jeong Seop Rhee for their scientific insights, discussions, and suggestions during progress of the project.

I wish to acknowledge my collaborators Prof. Johannes Hirrlinger, Dr. Sonja Wojcik, and Dr. Volker Eulenburg for their scientific discussions on this project.

Many thanks to Anja Annett Grützner for excellent technical assistance. Beyond this, she deserves a special regard for being a nice colleague. I also extend thank to all staffs in animal facilities, mechanical workshop and electronic workshop for their respective assistance.

To lab members Christian, Michal, and Mahmoud, I am grateful for their scientific and nonscientific discussions and for making a lively environment in the lab. Further, I acknowledge the friendly discussion with Christiane, Blinda, Oliwia, Karolina, Jonathan and Michael in coffee breaks.

To DFG and GGNB, I extend thank for financial support because without their support, I could not have carried out my thesis work and wonderful life experience in Germany.

For scientific and English comments on earlier version of my thesis, I thank to Michal Fortuna, Saju Balakrishnan, Christian Schnell, Christiane Menzfeld, Oliwia Janc and Zeeshan Ahmad.

To all my friends here and abroad, I am thankful for your care, love and regular contact, without which it would not be possible for me to arrive at this juncture.

To my parents, brother, and sisters, I am indebted for their prayers and support throughout my life. Finally, I acknowledge love and support of my wonderful wife Shaima who came Göttingen to accompany me and we have been blessed with daughter Safia. Thank you for your love, care and support. 